\title{
Starspot photometry with robotic telescopes
}

\section{Continuous $U B V$ and $V(R I)_{\mathrm{C}}$ photometry of 23 stars in 1991-1996}

\author{
K.G. Strassmeier ${ }^{1}$, J. Bartus ${ }^{1,2}$, G. Cutispoto ${ }^{3}$, and M. Rodonó ${ }^{3}$ \\ 1 Institut für Astronomie, Universität Wien, Türkenschanzstraße 17, A-1180 Wien, Austria \\ strassmeier@astro.ast.univie.ac.at \\ 2 Konkoly Observatory, Hungarian Academy of Sciences, H-1525 Budapest, Hungary \\ bartus@buda.konkoly.hu \\ 3 Catania Astrophysical Observatory, V.le A. Doria 6, I-95125 Catania, Italy \\ gcutispoto@alpha4.ct.astro.it,mrodono@alpha4.ct.astro.it
}

Received September 23; accepted December 16, 1996

\begin{abstract}
We report on the progress of our ongoing photometric monitoring program of spotted late-type stars with automatic photoelectric telescopes (APTs) on Mt. Hopkins in Arizona and on Mt. Etna in Sicily. We present 9250 differential $U B V$ and/or $V(R I)_{\mathrm{C}}$ observations for altogether 23 chromospherically active stars, singles and binaries, pre main sequence and post main sequence, taken between 1991 and 1996. The variability mechanism of our target stars is mostly rotational modulation by an asymmetrically spotted stellar surface. Therefore, precise rotational periods and their seasonal variations are determined using baselines between 3 years for HD 129333 to 34 years for V410 Tauri. We report the largest $V$ light-curve amplitude of any spotted star observed to date: 0.65 for V410 Tau in 1994-95. Long-term variations of the overall light levels of our target stars are sometimes of similar amplitude as the rotational modulation itself and are most likely caused by an analog of the solar 11-year spot cycle but mostly without a well-defined periodicity. For some of our target stars (HD 12545, HD 17433, EI Eri, V410 Tau, LQ Hya, and HD 106225) we estimate a probable cycle period. A complete light curve of the semi-regular S-type giant HR Pegasii is presented. All data are available via the WorldWideWeb ${ }^{1}$.
\end{abstract}

Key words: stars: activity - stars: late-type stars: rotation - binaries: spectroscopic - techniques: photometric

\footnotetext{
$1 \quad$ Data are available at the CDS via anonymous ftp.
}

\section{Introduction: Starspot photometry}

Photometry of late-type stars with inhomogeneous surface brightness distributions revealed distinct light-curve variations modulated with the stellar rotation period. Numerous papers dealt with this phenomenology since the early seventies, starting with the first interpretations by Kron (1947, 1952), Hoffmeister (1965), Chugainov (1966), Catalano \& Rodonó (1967) and Hall (1972) and finally culminating in the use of fully automatic telescopes for continuously monitoring RS CVn- and even solar-type stars (e.g. Henry et al. 1995a). Recently, Hall (1994) presented an update of the total number of known spotted stars and listed 357 entries including the chromospherically-active binaries from the catalog of Strassmeier et al. (1993a).

The physical phenomena behind such photometric modulations are starspots, i.e. local magnetic fields that are strong enough to prevent the overturning convective motion and thus the flow of energy from the stellar interior outwards to the surface and consequently appear as cool and therefore dark areas against an otherwise bright photosphere (Biermann 1941). The simple existence of starspots enables not only an accurate determination of the stellar rotation period, and eventually also differential rotation, but also to obtain some information about the surface temperature distribution and its intrinsic changes. We believe that these changes on the surface are a signature of the underlying dynamo process, itself rooting in the deepest convective layers of the star. Armed with long time series of photometric data one could try to detect analoga of the solar 11-year spot cycle in evolved stars or in pre-main sequence objects or even discover a stellar analog of the solar butterfly diagram. An automated approach is the key towards the needed time and phase coverage. 
Table 1. Program stars

\begin{tabular}{|c|c|c|c|c|c|c|c|c|}
\hline Star & $\begin{array}{l}V \\
\text { (mag) }\end{array}$ & Spectral type & $\begin{array}{l}\text { Binary? } \\
(\mathrm{SB} / \mathrm{S})\end{array}$ & $\begin{array}{l}P_{\text {orb }} \\
\text { (days) }\end{array}$ & $\begin{array}{l}<P_{\mathrm{phtm}}> \\
\text { (days) }\end{array}$ & $\begin{array}{l}v \sin i \\
\left(\mathrm{~km} \mathrm{~s}^{-1}\right)\end{array}$ & $\begin{array}{l}\text { Radius } \\
\left(R_{\odot}\right)\end{array}$ & Variable type \\
\hline HD 12545 (XX Tri) & 8.1 & K0III & SB1 & 23.98 & 24.30 & 17 & $\geq 8.2$ & RS CVn \\
\hline HD 17433 (VY Ari) & 6.9 & K3-4IV-V & SB1 & 13.20 & 16.42 & 6 & $\geq 1.95$ & $\mathrm{RS} \mathrm{CVn}$ \\
\hline HD 26337 (EI Eri) & 7.0 & G5IV & SB1 & 1.947 & 1.945 & 50 & $\geq 1.9$ & RS CVn \\
\hline HD 283518 (V410 Tau) & 11.0 & K4 & S & $\ldots$ & 1.872 & 77 & $\overline{3.0}$ & weak T Tauri \\
\hline HD 283750 (V833 Tau) & 8.2 & $\mathrm{~K} 5 \mathrm{~V}$ & SB1 & 1.788 & 1.797 & 6.3 & $\geq 0.22$ & BY Dra \\
\hline HD 282624 (SU Aur) & 8.9 & G2 & $\mathrm{S}$ & $\ldots$ & 2.98 & 66 & $\geq 3.9$ & class. T Tauri \\
\hline HD 31738 (V1198 Ori) & 7.1 & G5IV & SB2 & $?$ & $(4.59)$ & 19 & $\geq 1.7$ & $\mathrm{RS}$ CVn \\
\hline HD 31993 (V1192 Ori) & 7.5 & K2III & $\mathrm{S}$ & $\ldots$ & 6.78 & 31 & $\geq 4.1$ & single giant \\
\hline HD 39576 & 9.1 & G1V & $\mathrm{S}$ & $\ldots$ & 2.7 & 20 & $\geq 1.07$ & solar type \\
\hline HD 81410 (IL Hya) & 7.5 & K1III & SB1 & 12.90 & 12.73 & 24 & $\geq 6.0$ & RS CVn \\
\hline HD 82443 & 7.0 & $\mathrm{~K} 0 \mathrm{~V}$ & $\mathrm{~S}$ & $\ldots$ & 5.43 & 5 & $\geq 0.54$ & solar type \\
\hline HD 82558 (LQ Hya) & 7.8 & $\mathrm{~K} 2 \mathrm{~V}$ & $\mathrm{~S}$ & $\ldots$ & 1.606 & 28 & 0.94 & solar type \\
\hline HD 106225 (HU Vir) & 8.6 & K0III-IV & SB1 & 10.39 & 10.1 & 25 & $\geq 5.1$ & RS CVn \\
\hline HD 111812 (31 Com) & 4.9 & G0III & S & $\ldots$ & $?$ & 57 & $\bar{?}$ & single giant \\
\hline HD 112313 (IN Com) & 8.0 & G5III-IV & SB1 & $?$ & 5.9 & 67 & $\geq 7.8$ & $\mathrm{RS} \mathrm{CVn}$ \\
\hline HD 113816 & 8.3 & K0III & SB1 & 23.7 & 23.5 & $5-10$ & $\geq 2.3-4.6$ & RS CVn \\
\hline HD 116544 (IN Vir) & 9.2 & K2-3IV & SB1 & 8.19 & 8.23 & 23.5 & $\geq 3.8$ & RS CVn \\
\hline HD 117555 (FK Com) & 8.1 & G2III & S & $\ldots$ & 2.40 & 159 & $\geq 7.3$ & FK Comae \\
\hline HD 129333 (EK Dra) & 7.5 & G0-1V & $\mathrm{S}$ & $\ldots$ & 2.70 & 17.5 & 0.92 & solar type \\
\hline BD-08³999 (UZ Lib) & 9.3 & K0III & SB1 & 4.77 & 4.74 & 67 & $\geq 6.3$ & RS CVn \\
\hline HD 195040 (AT Cap) & 8.9 & K2III & SB1 & 23.21 & 23.2 & 19 & $\geq 10$ & RS CVn \\
\hline HD 202077 (BM Mic) & 8.3 & G6IV-V & S & $\ldots$ & 14.3 & $?$ & $\bar{?}$ & single subgiant \\
\hline HD 216489 (IM Peg) & 5.6 & K2II-III & SB1 & 24.65 & 24.4 & 24 & $\geq 12$ & RS CVn \\
\hline
\end{tabular}

Table 2. Number of observations

\begin{tabular}{lllllll}
\hline Variable & Comparison & Check & Time range & $n_{\text {T7 }}$ & $n_{\text {Pho }}$ & $n_{\text {Cat }}$ \\
& & & $2400000+$ & & & \\
\hline HD 12545 (XX Tri) & HD 12478 & SAO 55178 & $48648-50130$ & 302 & 259 & 58 \\
HD 17433 (VY Ari) & HD 17572 & HD 17361 & $48529-50137$ & $\ldots$ & 315 & 72 \\
HD 26337 (EI Eri) & HD 26409 & HD 25852 & $49284-50123$ & 5 & 270 & 23 \\
HD 283518 (V410 Tau) & HD 27159 & HD 27570 & $49612-50164$ & 134 & $\ldots$ & $\ldots$ \\
HD 283750 (V833 Tau) & HD 283749 & HD 29169 & $48957-49353$ & 231 & $\ldots$ & 25 \\
HD 282624 (SU Aur) & HD 31565 & HD 31305 & $49403-49443$ & 14 & $\ldots$ & \\
HD 31738 (V1198 Ori) & HD 31594 & HD 32263 & $49044-50174$ & 316 & $\ldots$ & $\ldots$ \\
HD 31993 (V1192 Ori) & HD 32073 & HD 32191 & $49023-50172$ & 286 & $\ldots$ & $\ldots$ \\
HD 39576 (IL Hya) & HD 39636 & SAO 170938 & $49023-50182$ & 271 & $\ldots$ & $\ldots$ \\
HD 81410 81904 & HD 80991 & $49023-50230$ & 440 & $\ldots$ & $\ldots$ \\
HD 82443 & HD 83098 & HD 83821 & $49393-50170$ & $\ldots$ & 142 & $\ldots$ \\
HD 82558 (LQ Hya) & HD 82447 & HD 82508 & $49044-50233$ & 445 & 40 & $\ldots$ \\
HD 106225 (HU Vir) & HD 106270 & HD 105796 & $48245-50263$ & 581 & 809 & $\ldots$ \\
HD 111812 (31 Com) & HD 111469 & HD 111395 & $49405-50263$ & 104 & 197 & $\ldots$ \\
HD 112313 (IN Com) & HD 112299 & HD 112706 & $49044-50263$ & 521 & $\ldots$ & 80 \\
HD 113816 (IN Vir) & HD 113449 & SAO 139146 & $49025-50263$ & 564 & $\ldots$ & $\ldots$ \\
HD 116544 (IN 117635 & HD 118830 & $49441-50263$ & 451 & $\ldots$ & 30 \\
HD 117555 (FK Com) & HD 117567 & HD 117876 & $49044-49892$ & 249 & $\ldots$ & 58 \\
HD 129333 (EK Dra) & HD 129390 & HD 129798 & $49398-50263$ & 210 & 112 & $\ldots$ \\
BD-08 3999 (UZ Lib) & SAO 140587 & SAO 140589 & $49022-50263$ & 688 & $\ldots$ & $\ldots$ \\
HD 195040 (AT Cap) & HD 195106 & SAO 189362 & $49082-50261$ & 393 & $\ldots$ & $\ldots$ \\
HD 202077 (BM Mic) & HD 202540 & HD 201695 & $49101-50263$ & 241 & $\ldots$ & $\ldots$ \\
HD 216489 (IM Peg) & HD 216635 & HD 216672 & $49297-50263$ & 218 & $\ldots$ & 111 \\
\hline
\end{tabular}

${ }^{1}$ after JD 2450000 we used HD 106332 as the check star.

${ }^{2}$ this is the variable star HR Peg $=$ HR 8714 (see discussion). 
In the past decade only few photometric studies were presented that tried to continuously monitor spotted stars and then model their intrinsic spot variations from one rotation to the next (e.g. Bartolini et al. 1983; Strassmeier et al. 1988; Jetsu et al. 1990; Olàh et al. 1991; Strassmeier \& Bopp 1992; Strassmeier et al. 1994a; Henry et al. 1995b; Rodonò et al. 1995; Olàh et al. 1996). One physical parameter that can be extracted from these data is the spot lifetime. For example, Henry et al. (1995b) observed individual spot lifetimes on $\lambda$ And and II Peg between 0.5 years and over 6 years, on $\sigma$ Gem between 0.2 and 3.5 years and on V711 Tau between 0.4 and 4 years from 15-19 years of photometric observations. Similar was found for two other active stars, VY Ari $=$ HD 17433 (Strassmeier \& Bopp 1992) and HR 7275 (Strassmeier et al. 1994a) or for HK Lac where polar activity centers persisted for over 30 years (Oláh et al. 1997). Similar timescales are encountered in RS CVn itself: from 45 years of photometric data Rodonó et al. (1995) found a cyclic change of the total spotted area with a period of 20 years and a spot minimum in 1993 and a maximum in 1984-88. Although spot lifetimes are not explicitly given in that paper we estimate a lower limit of about 0.4 years from their spot maps in 1991 and again in 1993. On the other hand, the G5IVbinary EI Eri exhibits spots with very short variability timescales, sometimes as short as several stellar rotations or about two weeks (Strassmeier 1990).

Obviously, photometric monitoring with a high duty cycle and for a long period in time is needed and the major boost for continuous starspot photometry came only with the availability of fully automatic photoelectric telescopes. Fairborn Observatory was the first to put such telescopes into operation in 1983 (Boyd et al. 1984) and a first series of papers was published by the then Vanderbilt/Fairborn group of D.S. Hall (Strassmeier \& Hall 1988a,b; Strassmeier et al. 1989a; Boyd et al. 1990). In the meantime altogether nine APTs, eight in the U.S. and one in Italy, are currently taking data on various types of objects (see several articles in Henry \& Eaton 191995). Repeated observations are also made at several manual observatories, most noticeably at ESO with the 50-cm size telescopes (Sterken 1983; Sterken et al. 1995; Cutispoto 1995). The results of all these photometric observations are series of light curves for about hundred spotted stars with coverages just interrupted by the annual seasons, the day-and-night cycle and, of course, unfavorable weather conditions and - rare - telescope failures.

In this paper we present new multicolor data from the Fairborn 0.75-m T7 APT (now part of the University of Vienna twin APT; see Strassmeier et al. 1997), the 0.25-m Phoenix-10 APT (Boyd et al. 1984) and from the Catania 0.8-m APT (Rodonó \& Cutispoto 1994). Altogether 23 program stars were observed in either $U B V$ or $V(R I)_{\mathrm{C}}$ or in both and also with varying degrees of time and phase coverage on the individual telescopes. Table 1 presents our target stars and their commonly known stellar parame- ters. A period analysis for all stars for each individual observing season as well as for the combined data gives a homogeneous set of stellar rotation periods and their seasonal variations. The long-term light curve behavior is examined together with additional, published photometry. Partially unpublished data by Schnell (1996), Oláh \& Jurcsik (1996), Jurcsik (1996), Skiff \& Nations (1991), Hall (1988), Barksdale (1990), and Petreshock (1996) are also used in the analysis in this paper.

Table 3. Problems encountered with the T7 APT

\begin{tabular}{llll}
\hline & Time & Influence & Cause \\
& $2449+$ & on data & \\
\hline A & $144-164$ & No $R$ and $I$ data & Filter wheel stuck at $V$ \\
B & $235-246$ & No $R$ and $I$ data & Filter wheel stuck at $V$ \\
C & $312-322$ & No $R$ and $I$ data & Filter wheel stuck at $V$ \\
D & $600-850$ & $\sigma_{\text {ext }}$ increased & Telescope out of focus \\
\hline
\end{tabular}

\section{Instrumentation and data quality}

\subsection{Filter systems}

Our new observations were obtained with three different automatic photoelectric telescopes (APT) in the years 1991-96: the 0.75-m Fairborn APT on Mt. Hopkins in Arizona, U.S.A. equipped with $V(R I)_{\mathrm{C}}$ filters matching the Johnson-Cousins system, the $0.25-\mathrm{m}$ Phoenix APT also on Mt. Hopkins but equipped with Johnson $U B V$, and the 0.8-m Catania APT on Mt. Etna in Sicily, Italy with $U B V$ filters for the Johnson system.

All measurements were made differentially with respect to a nearby comparison star. Table 2 identifies the comparison stars and the check stars and also gives the total number of obtained mean differential $V$ magnitudes per star and per telescope $\left(n_{\mathrm{T} 7}\right.$ for the Fairborn APT, $n_{\text {Pho }}$ for the Phoenix-10, and $n_{\text {Cat }}$ for the Catania APT). Both APTs on Mt. Hopkins observed each program star differentially with respect to a comparison star and a check star in the following sequence: $\mathrm{N}-\mathrm{CK}-\mathrm{S}-\mathrm{C}-\mathrm{V}-\mathrm{C}-$ $\mathrm{V}-\mathrm{C}-\mathrm{V}-\mathrm{C}-\mathrm{S}-\mathrm{CK}$, where $\mathrm{N}$ is a bright navigation star, CK is the check star, $\mathrm{C}$ the comparison star, $\mathrm{S}$ the sky background usually between the comparison and the variable star, and $\mathrm{V}$ the variable itself. One entry for $n$ in Table 2 thus compromises at least three individual V - C readings.

In order to eliminate the datapoints grossly in error we applied a statistical procedure that eliminated all data with an internal standard deviation greater than $\pm 00^{\mathrm{m}} 02$ as well as data that deviated from the rest by at least $3 \sigma_{\text {ext }}$ (see, e.g., Hall et al. 1986). While the 0.02-mag filtering excluded between $5-30 \%$ of the whole data for a particular star the number of mean magnitudes excluded by the 
$\mathrm{N}-\mathrm{CK}-\mathrm{C}-\mathrm{V}-\mathrm{V}-\mathrm{V}-\mathrm{C}-\mathrm{V}-\mathrm{V}-\mathrm{V}-\mathrm{C}-\mathrm{CK}-\mathrm{N}$ with the same meaning for the symbols as above. The sky background is measured at a fixed position near each star. Each magnitude on the variable star consists of six readings, compared to four with the other APTs. Integration time in $U, B$, and $V$ was set to 15,10 , and $10 \mathrm{~s}$, respectively. The typical standard deviations of the averaged $\mathrm{V}-\mathrm{C}$ and $\mathrm{V}-\mathrm{CK}$ magnitudes for stars brighter than $V \approx 9$ mag are of the order of $0.015,0.010$, and $0.007 \mathrm{mag}$ for $U, B$, and $V$, respectively. The accuracy of the standard $V$ magnitude is $0.01 \mathrm{mag}$, for $U-B$ about $0.02 \mathrm{mag}$, and for $B-V$ about 0.01 mag.

\section{Results}

All of our program stars except 31 Comae are known variable stars and were chosen on the grounds that they are part of an ongoing campaign on Doppler imaging or another spectroscopic project, or because there was simply a lack of photometry. Table 1 lists the program stars and summarizes their most relevant properties taken from the second edition of the Catalog of Chromospherically Active Binary Stars (Strassmeier et al. 1993a) or, if just recently discovered or in case of a single star, from literature quoted in Sect. 4 in this paper.

\subsection{Photometric periods}

Table 4 summarizes the results of our period analysis. We used a menu-driven Fortran program that performs a multiple frequency search through minimization of the residuals with a Fourier option (Kollath 1990, but see also Breger 1990). Errors are estimated from the width of the frequency peak at $\chi_{\min }^{2}+\left(\chi_{\min }^{2} /(n-m)\right)$ where $n$ is the number of data points and $m$ the number of parameters $(n-m$ is then the number of the degrees of freedom; $m=4$ in our case). For details we refer to Bevington (1969). Figure 1 shows the periodogram for the combined and seasonal $V$ data of HD 12545 and FK Com and the respective window functions from the various observing modes over the years. This figure is representative for the periodogram analysis in this paper. All photometric periods in Table 4 were obtained from the $V$ data only, mostly because of the particular good time coverage in $V$ and included our own as well as many of the previously published data listed in Table 5. Some of the data have been prewhitened and if so this is indicated in Sect. 4. No systematic zero point adjustments were necessary.

The periods designated $<P_{\mathrm{phtm}}>$ in Table 4 are the long-term average photometric periods computed from the full data sets including the literature data and probably best represent the stellar rotation period. All light curves in this paper are phased with this period unless otherwise noted. The corresponding zero point in time, $T_{0}$, is the arbitrary time of the first observation in each data set. Deviations of the seasonal periods from this long-term av- erage can be directly associated with differential surface rotation in case the seasonal data coverage permits a sufficiently precise period determination.

For those binaries in our sample where the rotation is sufficiently synchronized to the orbital motion and only few data were available we chose the orbital period instead of the long-term photometric period to phase the data. These stars are marked in Table 4 . An orbital period is usually known with higher precision and accuracy than a photometric period and is thus a good timekeeper. However, there is some theoretical support that the convective motion in the outer layers of close late-type binary components is governed by the orbital angular momentum (Schrijver \& Zwaan 1991) and care must be exercised when data from long intervals in time are phased together. Most eclipsing RS CVn binaries show indeed significant orbitalperiod variations. The prototype RS CVn, for example, exhibits $\mathrm{O}-\mathrm{C}$ variations of up to 0.2 days in about 40 years (Rodonó et al. 1995).

\subsection{Long-term brightness variations}

It is now a well-established fact that solar-type stars exhibit the same long-term cycles in their photospheric output as originally discovered from the chromospheric Ca II H\&K emission (e.g. Baliunas \& Soon 1995; Gray et al. 1996; 1992), thus strengthening the solar-stellar connection. For evolved stars, however, a study of variability in a sample of $\mathrm{G}$ and $\mathrm{K}$ giants by Choi et al. (1995) showed only one clear detection $(\delta \mathrm{CrB})$ of a rotationally modulated signal from both $V$-band photometry and CaII$S$-index spectrophotometry. It is thus not surprising that long-term changes in the mean brightness of RS CVn-type stars are not a straightforward tool to detect spot cycles similar to the Sun's 11-year cycle despite the now rapidly growing data base. There are basically three reasons for this: first, very active evolved and, compared to the Sun, heavily spotted stars might not have such strictly cyclic behavior and/or, second, their long-term cycles are of relatively small amplitude and are masked by short-term irregularities such as dynamo-unrelated redistributions of the starspot asymmetry (note that only the asymmetric part contributes to the light-curve amplitude) and/or, third, their cycle periods are much longer than those found for solar-type stars ( 7 to 21 years according to Baliunas \& Soon 1995).

Convincing observational evidence for the existence of long-term and cyclic brightness variations in very-active late-type dwarf stars comes from the photographic magnitudes measured off archival plate collections. Hartmann et al. (1981) discovered a $\approx 60$-year cycle with an amplitude of 0.5 mag for V833 Tau (K5V) from plates dating back to the early 1900's. A similar periodicity with an amplitude of 0.3 mag was found for BY Dra, the prototype BY-Draconis system consisting of two late K-dwarf stars (Phillips \& Hartmann 1978). Just recently, Bondar (1995) 
Table 4. Photometric periods

\begin{tabular}{|c|c|c|c|c|c|c|c|c|}
\hline \multirow[t]{2}{*}{ Star } & \multicolumn{5}{|c|}{ Observational seasons $P_{\mathrm{phtm}}$} & \multirow{2}{*}{$\begin{array}{l}T_{0} \\
\left(\begin{array}{lll}2 & 400 & 000+\end{array}\right)\end{array}$} & \multicolumn{2}{|c|}{ Long-term average $<P_{\mathrm{phtm}}>$} \\
\hline & 91-92 & $92-93$ & $93-94$ & 94-95 & $95-96$ & & (days) & Time range \\
\hline \multirow[t]{2}{*}{ HD 12545} & 25.32 & 24.08 & 24.69 & 24.01 & 23.87 & \multirow[t]{2}{*}{46370.0} & 24.0801 & \multirow[t]{2}{*}{$1985-96$} \\
\hline & \pm 0.64 & \pm 0.09 & \pm 0.10 & \pm 0.11 & \pm 0.02 & & \pm 0.0035 & \\
\hline \multirow[t]{2}{*}{ HD 17433} & 16.515 & 17.29 & 16.242 & 16.308 & 16.318 & \multirow[t]{2}{*}{40029.0} & 16.1996 & \multirow[t]{2}{*}{$1973-96$} \\
\hline & \pm 0.055 & \pm 0.14 & \pm 0.041 & \pm 0.075 & \pm 0.054 & & \pm 0.0029 & \\
\hline \multirow[t]{2}{*}{ EI Eri } & 1.9352 & 1.9419 & 1.9444 & 1.9436 & 1.9456 & \multirow[t]{2}{*}{44129.0} & 1.952717 & \multirow[t]{2}{*}{$1980-96$} \\
\hline & \pm 0.0009 & \pm 0.0016 & \pm 0.0013 & \pm 0.0076 & \pm 0.0028 & & \pm 0.000031 & \\
\hline \multirow[t]{2}{*}{ V410 Tau } & 1.87182 & 1.87159 & $\ldots$ & 1.87089 & 1.87299 & \multirow[t]{2}{*}{37962.0} & 1.872025 & \multirow[t]{2}{*}{$1962-96$} \\
\hline & \pm 0.00040 & \pm 0.00060 & $\ldots$ & \pm 0.00027 & \pm 0.00060 & & \pm 0.000011 & \\
\hline \multirow[t]{2}{*}{ V833 Tau } & 1.8522 & 1.7737 & 1.8015 & 1.7963 & 1.8982 & \multirow[t]{2}{*}{45618.0} & 1.791601 & \multirow[t]{2}{*}{$1984-96$} \\
\hline & \pm 0.0089 & \pm 0.0031 & \pm 0.0023 & \pm 0.0024 & \pm 0.0034 & & \pm 0.000064 & \\
\hline \multirow[t]{2}{*}{ SU Aur } & $\ldots$ & $\ldots$ & 2.742 & $\ldots$ & $\ldots$ & \multirow[t]{2}{*}{41952.0} & 2.69935 & \multirow[t]{2}{*}{ 1973-94 } \\
\hline & $\ldots$ & $\ldots$ & \pm 0.045 & $\ldots$ & $\ldots$ & & \pm 0.00038 & \\
\hline HD $31738^{1}$ & $\ldots$ & $\ldots$ & $\ldots$ & $\ldots$ & $\ldots$ & $\ldots$ & $\ldots$ & 1979-96 \\
\hline HD 31993 & $\ldots$ & $\ldots$ & 27.78 & 28.09 & 27.62 & 43856.0 & 28.1246 & $1979-96$ \\
\hline & $\ldots$ & $\ldots$ & \pm 0.27 & \pm 0.31 & \pm 0.30 & & \pm 0.0079 & \\
\hline HD 39576 & 2.796 & 2.286 & 2.2655 & $\ldots$ & 2.2806 & 48636.0 & 2.26883 & 1992-96 \\
\hline & \pm 0.092 & \pm 0.019 & \pm 0.0046 & $\ldots$ & \pm 0.0047 & & \pm 0.00032 & \\
\hline HD 81410 & $\ldots$ & 12.669 & 12.723 & 12.864 & 12.826 & 40988.0 & 12.78642 & $1971-96$ \\
\hline & $\ldots$ & \pm 0.032 & \pm 0.054 & \pm 0.019 & \pm 0.019 & & \pm 0.00082 & \\
\hline HD 82443 & $\ldots$ & $\ldots$ & 5.492 & 5.413 & 5.409 & 47567.0 & 5.40482 & 1989-96 \\
\hline & $\ldots$ & $\ldots$ & \pm 0.042 & \pm 0.023 & \pm 0.013 & & \pm 0.00053 & \\
\hline LQ Hya & 1.60769 & 1.6154 & 1.60555 & 1.6033 & 1.60689 & 45275.0 & 1.600881 & 1984-96 \\
\hline & \pm 0.00048 & \pm 0.0045 & \pm 0.00067 & \pm 0.0014 & \pm 0.00072 & & \pm 0.000028 & \\
\hline HD 106225 & 10.353 & 10.424 & 10.102 & 10.327 & 10.448 & 45110.0 & 10.4181 & $1982-96$ \\
\hline & \pm 0.040 & \pm 0.013 & \pm 0.035 & \pm 0.026 & \pm 0.013 & & \pm 0.0011 & \\
\hline $31 \mathrm{Com}^{1}$ & $\ldots$ & $\ldots$ & $\ldots$ & $\ldots$ & $\ldots$ & $\ldots$ & $\ldots$ & 1984-96 \\
\hline IN Com & $\ldots$ & 5.903 & 5.903 & 5.958 & 5.9108 & 45444.0 & 5.93474 & $1983-96$ \\
\hline & $\ldots$ & \pm 0.029 & \pm 0.016 & \pm 0.021 & \pm 0.0071 & & \pm 0.00021 & \\
\hline HD 113816 & $\ldots$ & 24.05 & 23.17 & 23.51 & 24.50 & 49025.0 & 23.674 & 1993-96 \\
\hline & $\ldots$ & \pm 0.40 & \pm 0.21 & \pm 0.31 & \pm 0.42 & & \pm 0.036 & \\
\hline IN Vir & $\ldots$ & $\ldots$ & 8.281 & 8.155 & 8.1500 & 47949.0 & 8.18833 & $1990-96$ \\
\hline & $\ldots$ & $\ldots$ & \pm 0.018 & \pm 0.012 & \pm 0.0066 & & \pm 0.00080 & \\
\hline FK Com & 2.4064 & 2.4035 & 2.40211 & 2.3896 & $\ldots$ & 39253.0 & 2.397696 & $1966-95$ \\
\hline & \pm 0.0057 & \pm 0.0021 & \pm 0.00086 & \pm 0.0023 & $\ldots$ & & \pm 0.000029 & \\
\hline HD 129333 & $\ldots$ & $\ldots$ & 2.796 & 2.6023 & 2.6033 & 49403.0 & 2.60498 & 1994-96 \\
\hline & $\ldots$ & $\ldots$ & \pm 0.026 & \pm 0.0011 & \pm 0.0036 & & \pm 0.00030 & \\
\hline UZ Lib & $\ldots$ & 4.7655 & 4.7343 & 4.7659 & 4.654 & 41396.0 & 4.752264 & $1972-96$ \\
\hline & $\ldots$ & \pm 0.0063 & \pm 0.0066 & \pm 0.0026 & \pm 0.024 & & \pm 0.000090 & \\
\hline HD $195040^{2}$ & $\ldots$ & 22.32 & 11.569 & 11.542 & 11.64 & 44355.47 & 11.5937 & 1979-96 \\
\hline & $\ldots$ & \pm 0.23 & \pm 0.021 & \pm 0.069 & \pm 0.16 & & \pm 0.0030 & \\
\hline HD 202077 & $\ldots$ & 14.67 & 14.65 & 15.37 & 14.69 & 44071.0 & 14.7957 & $1979-96$ \\
\hline & $\ldots$ & \pm 0.13 & \pm 0.59 & \pm 0.18 & \pm 0.67 & & \pm 0.0017 & \\
\hline IM Peg & $\ldots$ & $\ldots$ & 25.19 & 24.108 & 24.498 & 43734.0 & 24.4936 & $1978-96$ \\
\hline & $\ldots$ & $\ldots$ & \pm 0.39 & \pm 0.081 & \pm 0.030 & & \pm 0.0024 & \\
\hline
\end{tabular}

${ }^{1}$ No short-term light variability detected. See Discussion.

${ }^{2}$ Double-humped light curve. Seasonal data are phased with the orbital period of 23.206 days.

discovered an even larger amplitude (0.8 - $1.0 \mathrm{mag})$ longterm variable: $\mathrm{PZ}$ Mon with a cycle period of 50 years. Again, PZ Mon is a dK2e-type main-sequence flare star similar to V833 Tauri. But what about evolved stars?

A baseline of 15-19 years of photometry of the four RS CVn binaries $\lambda$ And, $\sigma$ Gem, II Peg and V711 Tau was collected and analysed by Henry et al. (1995b). Long-term variability with a periodic behavior in mean brightness was discovered in all four of them: $11.1 \mathrm{yr}$ for $\lambda$ And, $8.5 \mathrm{yr}$ for $\sigma$ Gem, $11 \mathrm{yr}$ for II Peg, and $16 \mathrm{yr}$ for V711 Tau. We may note, however, that these periodicities are not so obviously apparent from a plot of the long-term 
lightcurves because of the drastly variable rotational modulation in these stars but the existence of long-term variations seems well established. An even longer baseline in time (45 years) is available for RS CVn itself, a K2 subgiant in a 4.8-day orbit. Rodonó et al. (1995) found that the total area covered by spots shows a cyclic variation with a period of 20 years. Obviously, cycle periods in active RS CVn-type stars can be of the same order than those in solar-type stars but tend to be, on average, somewhat longer. Therefore, one of our goals in this paper is to built up a sufficiently long data base that enables the detection of such variations - periodic or not.

Figures 2 through 24 present all currently available $V$-band photometry for our program stars. Supplemental data are taken from the literature and are summarized in Table 5. Literature data that were available in numerical form are plotted as individual points, normally representing the average of three individual readings, while published light curves without numerical data are entered as vertical bars indicating their maximum and minimum brightness. Note that Fig. 25 shows the long-term light and color curves for the check star of IM Peg (=HR Peg).

\subsection{Seasonal light curves}

Figures 26 through 48 present the photometric data for the stars in Table 1. All of the individual figures are grouped into observational seasons such that one $V$-band plot versus Julian date (JD) appears on the upper left side and a phase plot of the entire season's data on the upper right side of each graph. The lower panels show the $B-V$ and/or the $V-I_{\mathrm{C}}$ colors, whenever observed. Individual symbols are identified in the respective panels. The season is indicated at the top of each panel. Furthermore, data from the different APTs as well as data taken from the literature are identified with separate symbols as indicated. We emphasize that the sometimes large scatter in the phase plots is almost exclusively due to intrinsic light curve changes and not due to instrumental scatter.

\section{Discussion}

The following discussion is intended to draw attention to some special technical circumstances or special features in the light-curve behavior of our program stars but is not supposed to be a summary of all relevant literature data. For previously published photometry we refer to Table 5 but will try to comment on the most relevant and most recent papers in the text. For a complete listing of all available literature on a particular star we recommend to consult the SIMBAD data base at CDS Strasbourg.

HD 12545 (XX Tri). This is the RS CVn star with the previously largest light-curve amplitude (0.60 mag; Nolthenius 1991). Consequently, it attracted several follow-up studies (see Table 5) although its light variability was already known since 1985 (Skiff \& Nations
Table 5. Published light curves

\begin{tabular}{|c|c|c|}
\hline Star & Epoch & Reference \\
\hline \multirow[t]{5}{*}{ HD 12545} & $46370-47947$ & Skiff \& Nations (1991) \\
\hline & $46654-46882$ & Hooten \& Hall (1990) \\
\hline & $48148-48323$ & Nolthenius (1991) \\
\hline & $48502-48691$ & Strassmeier \& Oláh (1992) \\
\hline & $48171-49708$ & Hampton et al. (1996) \\
\hline \multirow[t]{5}{*}{ HD 17433} & $42029-42344$ & Chugainov (1976) \\
\hline & $45948-45973$ & Eaton \& Poe (1986) \\
\hline & $46014-46761$ & Bopp et al. (1989) \\
\hline & $47065-47213$ & Strassmeier \& Bopp (1992) \\
\hline & $1992.7,1993.7$ & Alekseev (1996) \\
\hline \multirow[t]{12}{*}{ EI Eri } & $44129-44974$ & Lloyd-Evans \& Koen (1997) \\
\hline & $44287-44660$ & Fekel et al. (1982) \\
\hline & $44520-44660$ & Bopp et al. (1983) \\
\hline & $45260-45350$ & Hall et al. (1987) \\
\hline & $45304-45400$ & Baliunas et al. (1983) \\
\hline & $45700-46483$ & Strassmeier et al. (1989a) \\
\hline & $46821-47232$ & Strassmeier (1990) \\
\hline & $47129-47143$ & Hooten et al. (1989) \\
\hline & $47112-47602$ & Hall (1996) \\
\hline & $47480-47511$ & Barksdale (1990) \\
\hline & $1988.0-90.0$ & Rodonó \& Cutispoto (1992) \\
\hline & $47869-47888$ & Cutispoto (1995) \\
\hline \multirow[t]{12}{*}{ V410 Tau } & $37962-41734$ & Romano (1975) \\
\hline & $42469-44636$ & Rössiger (1981) \\
\hline & $44173-44176$ & Herbig \& Goodrich (1986) \\
\hline & $44227-44659$ & Rydgren et al. (1984) \\
\hline & $44897-44904$ & Rydgren \& Vrba (1983) \\
\hline & $45641-46862$ & Vrba et al. (1988) \\
\hline & $46012-46150$ & Holtzman et al. (1986) \\
\hline & $46044-46796$ & Bouvier et al. (1988) \\
\hline & $46432-47940$ & Herbst et al. (1994) \\
\hline & $47082-47612$ & Herbst (1989) \\
\hline & 1993 & Grankin (1994) \\
\hline & $46660-48935$ & Petrov et al. (1994) \\
\hline \multirow[t]{4}{*}{ V833 Tau } & $37699-45674$ & Eggen (1984) \\
\hline & $44136-44524$ & Hartmann et al. (1981) \\
\hline & $45618-47929$ & Oláh \& Pettersen (1991) \\
\hline & $48151-49066$ & Oláh \& Jurcsik (1996) \\
\hline \multirow[t]{11}{*}{ SU Aur } & $41953-42006$ & Rydgren et al. (1976) \\
\hline & $42357-42363$ & Cohen \& Schwartz (1976) \\
\hline & $39095-44637$ & Rydgren et al. (1984) \\
\hline & $44860-45399$ & Herbst et al. (1983) \\
\hline & $45282-45313$ & Gahm et al. (1993) \\
\hline & $45979-46022$ & Holtzman et al. (1986) \\
\hline & $46044-46796$ & Bouvier et al. (1988) \\
\hline & $46313-47605$ & Herbst et al. (1994) \\
\hline & $46313-46493$ & Herbst et al. (1987) \\
\hline & $47162-47174$ & Herbst \& Koret (1988) \\
\hline & $48212-48272$ & Bouvier et al. (1993) \\
\hline \multirow[t]{5}{*}{ HD 31738} & $43856-44967$ & Lloyd-Evans \& Koen (1987) \\
\hline & $44600-45400$ & Hall et al. (1986) \\
\hline & $45971-46505$ & Strassmeier et al. (1989a) \\
\hline & $47121-47434$ & Hall (1988) \\
\hline & $47869-47887$ & Cutispoto $(1995)$ \\
\hline \multirow[t]{2}{*}{ HD 31993} & $43856-44967$ & Lloyd-Evans \& Koen (1987) \\
\hline & $47417-47434$ & Hooten \& Hall (1990) \\
\hline HD 39576 & $48636-48644$ & Strassmeier et al. (1992) \\
\hline
\end{tabular}




\begin{tabular}{|c|c|c|}
\hline$\overline{\text { Star }}$ & Epoch & Reference \\
\hline \multirow[t]{12}{*}{ HD 81410} & $40988-41503$ & Eggen (1973) \\
\hline & $43860-45359$ & Lloyd-Evans \& Koen (1987) \\
\hline & $44363-44738$ & Collier-Cameron (1987) \\
\hline & $44608-44678$ & Raveendran et al. (1982) \\
\hline & 1988 & Mekkaden \& Sinachop. (1988) \\
\hline & $46066-49390$ & Manfroid et al. (1991) \\
\hline & $47129-47143$ & Pallavicini et al. (1993) \\
\hline & $47148-48077$ & Sterken et al. (1993) \\
\hline & $47548-47595$ & Cutispoto (1993) \\
\hline & $47869-47888$ & Cutispoto (1995) \\
\hline & $48189-48658$ & Manfroid et al. (1995) \\
\hline & $48957-49390$ & Sterken et al. (1995) \\
\hline \multirow[t]{2}{*}{ HD 82443} & $47567-47674$ & Messina \& Guinan (1996) \\
\hline & $49638-49717$ & Henry et al. (1995a) \\
\hline \multirow[t]{8}{*}{ LQ Hya } & $45275-45779$ & Fekel et al. (1986) \\
\hline & $45699-45734$ & Eggen (1984) \\
\hline & $46023-46529$ & Strassmeier \& Hall (1988b) \\
\hline & $47133-47143$ & Cutispoto (1991) \\
\hline & $47548-47593$ & Cutispoto (1993) \\
\hline & $47881-48760$ & Jetsu (1993) \\
\hline & $47949-47961$ & Cutispoto (1996) \\
\hline & $48270-48300$ & Strassmeier et al. (1993b) \\
\hline \multirow[t]{6}{*}{ HD 106225} & $45110-45153$ & Fekel et al. (1984) \\
\hline & $46832-46841$ & Cutispoto (1990) \\
\hline & $47548-47594$ & Cutispoto (1993) \\
\hline & $47949-47961$ & Cutispoto (1996) \\
\hline & $47987-48437$ & Hall \& Henry (1992) \\
\hline & $48270-48433$ & Strassmeier (1994) \\
\hline \multirow[t]{3}{*}{$31 \mathrm{Com}$} & $45722-46603$ & Strassmeier \& Hall (1988a) \\
\hline & $47163-47232$ & Hall (1988) \\
\hline & $49372-49521$ & Jasniewicz et al. (1996) \\
\hline \multirow[t]{9}{*}{ IN Com } & $45444-45525$ & Schnell \& Purgathofer (1983) \\
\hline & $45674-47288$ & Schnell (1996) \\
\hline & $1985,1986-89$ & Malasan et al. (1991) \\
\hline & $46883-47305$ & Noskova (1989) \\
\hline & $1988.4,1989.7$ & Bond \& Livio (1990) \\
\hline & $47604-47691$ & Jasniewicz et al. (1994) \\
\hline & $48979-49120$ & Jurcsik (1996) \\
\hline & $49100-49107$ & Kuszawska \& Mikolaj. (1993) \\
\hline & $49372-49521$ & Jasniewicz et al. (1996) \\
\hline HD 113816 & $49415-49528$ & Henry et al. (1995a) \\
\hline IN Vir & $47949-47961$ & Cutispoto et al. (1996) \\
\hline \multirow[t]{14}{*}{ FK Com } & $39253-39346$ & Chugainov (1966) \\
\hline & $42126-42223$ & Chugainov (1976) \\
\hline & $42829-42837$ & Rucinski (1981a) \\
\hline & $43624-43664$ & Olsen $(1980)$ \\
\hline & $44651-44661$ & Morris \& Milone (1983) \\
\hline & $45032-45207$ & Dorren et al. (1984) \\
\hline & $45045-48101$ & Jetsu et al. (1993) \\
\hline & $45116-45139$ & Bohusz \& Udalski (1983) \\
\hline & $45376-45388$ & Holtzman \& Nations (1984) \\
\hline & $45351-45501$ & Cellino et al. (1986) \\
\hline & $45466-45861$ & Huovelin et al. (1987) \\
\hline & $45796-45802$ & Oja (1985) \\
\hline & $45790-45803$ & Eaton $(1985)$ \\
\hline & $47292-47303$ & Heckert \& Maloney (1992) \\
\hline
\end{tabular}

\begin{tabular}{lll}
\hline Star & Epoch & Reference \\
\hline FK Com & 1990.4 & Marchev \& Kjurkchieva (1993) \\
& $48025-48403$ & Heckert et al. (1992) \\
& $48754-48838$ & Heckert (1993a) \\
& $49034-49120$ & Oláh \& Jurcsik (1996) \\
& $49804-49833$ & Petreshock et al. (1995) \\
HD 129333 & $46567-46588$ & Dorren \& Guinan (1994) \\
& 1991.4 & Chugainov et al. (1991) \\
& $49377-49410$ & Scheible \& Guinan (1994) \\
UZ Lib & 1930 & Parenago (1931) \\
& $41396-41501$ & Wisniewski (1973) \\
& 1977.3 & Hoffmann (1980) \\
& $43944-43957$ & Rucinski (1981b) \\
& $45423-45485$ & Bopp et al. (1984) \\
& 1983.2 & Nations \& Holtzman (1987) \\
& $46166-46628$ & Grewing et al. (1989) \\
& $1988.4,1989.5$ & Heckert \& Hickman (1991) \\
& $1990.4,1991.3$ & Heckert (1992) \\
& $48754-48844$ & Heckert (1993b) \\
& $49556-49564$ & Paunzen \& Strassmeier (1996) \\
& $43785-45166$ & Lloyd-Evans \& Koen (1987) \\
& $44088-44161$ & Collier-Cameron (1987) \\
& $44461-44485$ & Fekel et al. (1986) \\
HM Peg 195040 & $44071-44912$ & Lloyd-Evans \& Koen (1987) \\
& $49528-49549$ & Strassmeier et al. (1994b) \\
& $40876-41580$ & Herbst (1973) \\
& $44049-44576$ & Eaton et al. (1983) \\
& $45620-46601$ & Percy \& Welch (1982) \\
& $45957-45973$ & Eaton (1985) \\
& $48502-48654$ & Oláh (1996) \\
& $48859-48979$ & Dempsey et al. (1996) \\
\hline & & \\
& & \\
& & \\
& &
\end{tabular}

1991) and independently since 1986 (Hooten \& Hall 1990). A preliminary orbit with a period of 24 days had already been entered into the tables of the CABS catalog and a definite orbit was published in the meantime by Bopp et al. (1993).

The $y$ magnitudes of the Strömgren photometry of Skiff \& Nations (1991) have been converted to Johnson $V$ magnitudes with the relation given in Olsen (1983): $V=y+0.015(b-y)-0.003$ and are plotted in Fig. 2. Ten years of $V$ data suggest that the light-curve amplitude varies more or less systematically showing a small amplitude every $2.7 \pm 0.2$ years and a large amplitude shifted in time by about one half of that period. If periodic we expect to see the next minimum at around JD 2450500 $(\approx 1997.0)$. The maximum amplitude in our new data was $0.48 \mathrm{mag}$ in $V, 0.12 \mathrm{mag}$ in $V-I$, and $0.06 \mathrm{mag}$ in $B-V$ in December 1995 but only $\approx 0.1 \mathrm{mag}$ in $V$ and $0.04 \mathrm{mag}$ in $V-I$ and $0.02 \mathrm{mag}$ in $B-V$ one and a half years earlier while the light and color curves showed two minima at that time. The seasonal period continuously increased between 1988 and 1992 but changed inconsistently thereafter 
and do not seem to relate with the light-curve amplitude. Periodograms of the full data set and the 1995/96 observing season are compared in Fig. 1.

HD 17433 (VY Ari). An extensive multiwavelength study of this star was presented by Bopp et al. (1989), finding it to be one of the most active binaries yet observed. The relatively sharp lines $\left(v \sin i=6 \mathrm{~km} \mathrm{~s}^{-1}\right)$ allowed the detection of a $2 \mathrm{kG}$ magnetic field with a filling factor of 66\%. Photometry from 1974 through 1991 was discussed in Strassmeier \& Bopp (1992) and a new value for the rotation period of 16.42 days was found. Since the orbit of HD 17433 is nearly circular and the orbital and rotational periods differ by approximately 30\%, HD 17433 represents the case of an asynchronously rotating star. Time-series starspot modeling of the 1988/89 and 1989/90 photometry revealed emergence and decay timescales for individual spots that likely scale with the area of the spots - as is the case with sunspots.

Our new data cover five seasons from 1991/92 on and, together with the literature data cited in Table 5, now span over 22 years (Fig. 3). However, there is a big data gap between 1975 and 1985, but the trend in the more recent $V$ light curves suggests a sinusoidal long-term brightness variation possibly periodic with minimum light occuring at around 1994.0 and maximum light at around 1987.0 ( $P \approx 14$ years). The seasonal periods are plotted in the lower panel of Fig. 3 and exhibit variations exceeding the 3- $\sigma$ level but - as for HD 12545 - do not indicate a long-term relation with the overall system brightness. The present data density does not allow a conclusive statement whether the photometric period is related to the amount of the rotationally modulated amplitude (see the lower panel in Fig. 3). The seasonal phase curves are plotted in Fig. 27 and show the maximum amplitude of $0.18 \mathrm{mag}$ in $V$ and $0.04 \mathrm{mag}$ in $B-V$ in 1993/94.

HD 26337 (EI Eri). EI Eri has been a prime target for Doppler imaging since the first application of this technique to spotted late-type stars in 1982. Doppler images can be found in Hatzes \& Vogt (1992), Strassmeier et al. (1991), and Strassmeier (1990). All of these images show a cool polar spot whose appendages cause a significant part of the light-curve variability and, if real, has been present since observations commenced in 1982 by Vogt and collaborators. The photometric period of EI Eri is close to 2 days (1.95) and observations from a single ground-based site take $\approx 30$ days to cover all rotational phases. This is doubly unfortunate because a continuous IUE-FES light curves for 85 hours obtained by J. Neff (see Strassmeier et al. 1989b) showed clear variations of the shape of the light curve, i.e. within two consecutive stellar rotation cycles.

Our new data are from seven observing seasons starting in late 1989 and the long-term plot in Fig. 4 now covers the past 16 years. Seasonal phase plots are shown in Fig. 28 and give an impression of how rapidly the lightcurve shapes may change from cycle to cycle. The largest amplitude was observed in 1993/94 and reached $0.15 \mathrm{mag}$ in $V$ and $0.03 \mathrm{mag}$ in $B-V$ by the end of the season. Most remarkably, however, is the sinusoidal long-term brightness variation with a likely period of around $11 \pm 1$ year and an average $V$ amplitude of 0.1 mag. Once again, the seasonal periods indicate no clear relation with the longterm brightness variation.

HD 283518 (V410 Tau). Despite its relative faintness of 11th mag this star is the most well observed weakline T Tauri star in the sky. Extensive photometry exists from various sources (see Table 5) and recently has been entered into a publicly available photometric data base for T Tauri stars by Herbst et al. (1994). This service is gratefully acknowledged here! The most recent photometric period determination, and probably the best so far, has been that of Petrov et al. (1994) who found $1.872095 \pm 0.000022$ days. V410 Tau is also a prime target for Doppler imaging and four maps exist to date (Strassmeier et al. 1994d; Joncour et al. 1994; Hatzes 1995; Rice \& Strassmeier 1996). From a photometrists point of view it is especially interesting to note that our Doppler images reveal hot and cool spots coexisting on the photospheric surface. A fit to a light curve with a simple geometric two-spot model with only cool spots would, therefore, result in very uncertain if not completely spurious spot longitudes and areas. It seems that spot distributions in general are much more complicated than what is suggested from continuum light curves alone.

Our new data are from two observing seasons 19941996 and bring up the total time coverage to 34 years (Fig. 5). The seasonal phase plots are shown in Fig. 29. We emphasize that our observations in 1994-95 caught V410 Tau with an amplitude of 0.65 in $V$. This is the largest amplitude ever observed on any spotted star and the corresponding surface feature must cover almost an entire hemisphere. This could even affect the star's hydrostatic equilibrium in the outer regions because simple energy redistribution within the convection zone might not be sufficient any more to store away the "missing flux" blocked by such a huge spot. Since the accompanying $V-I$ color curve also shows the enormous amplitude of $0^{\mathrm{m}} 18$ and is in phase with the $V$ light curve we believe that the amplitudes are indeed due to temperature inhomogeneities on the stellar surface.

The long-term trend in Fig. 5 is very similar to that observed for HD 12545, now being the second largest amplitude star with a maximum $V$ amplitude of 0.60 . Both stars' average light level remain more or less constant and V410 Tauri also shows the same - possibly cyclic - change of the lightcurve amplitude but with a period between 6-7 years compared to $\approx 2.7 \mathrm{yr}$ for HD 12545 . Again, there is an obvious lack of correlation between the seasonal photometric period and the overall light-curve amplitude.

HD 283750 (V833 Tau). Oláh \& Pettersen (1991) and Guinan \& McCook (1991) have been observing this star since around 1983 and Eggen (1994) discovered its 
light variability from observations in 1962. The long-term variability was discovered by Hartmann et al. (1981) who found a very distinct cycle with a period of 60 years and an amplitude of around $\Delta m_{\mathrm{pg}} \approx 0.7 \mathrm{mag}$. Recently, Bondar (1995) extended the database with additional archival photographic magnitudes to a time range from 1899 to 1990 and confirmed the earlier cycle period. In November 1993 Guinan (1996) detected a huge flare in all five Johnson bandpasses that reached an amplitude of $0.5 \mathrm{mag}$ in $U$ and was still visible in $I$. Earlier, Oláh \& Pettersen (1991) suggested the existence of a long-lived polar spot due to the low inclination of the rotation axis of $\approx 20^{\circ}$ and the continuous light-curve variations on all accessable time scales.

Three seasons of photometric data and part of a third were obtained so far. These data are compared with other photoelectric data from the literature in Fig. 6. V833 Tau shows a long-term variation of $0.25 \mathrm{mag}$ between 1988 and 1996 which is at least twice as large as the amplitude due to rotational modulation. Therefore, periodogram analysis of the whole data set was carried out from prewhitened data by removing all frequencies longer than 270 days. The best period found was 1.791601 days (Table 4) very close to the orbital period of 1.788 days. Figure 30 shows the seasonal plots.

HD 282624 (SU Aur). SU Aurigae seems to be an intermediate case between a weak-lined T Tauri star and a classical $\mathrm{T}$ Tauri star because its photospheric spectrum is clearly seen but still slightly veiled (e.g. Giampapa et al. 1993; Basri et al. 1995). Despite the veiling, Petrov et al. (1995) presented a photospheric Doppler image that shows predominantly cool spots along the stellar equator. The true rotation period of SU Aur has been most unusual - determined from $\mathrm{H} \alpha$ line profile variations and is $2.98 \pm 0.4$ days (Giampapa et al. 1993). We note that Petrov et al. (1995) used a period of 3.094 days for phasing their spectroscopic data because earlier photometry by Herbst et al. (1987) caught the star in a stage when the light curve was double-humped and thus the best-fit period (1.55 days) was only one-half the true rotation period. Further photometry from various sources, partially even unpublished, is available from the T-Tauri data base of Herbst et al. (1994).

We have only one observing season of photometry of SU Aur. Figure 7 shows all available $V$-band photometry over the past 20 years. Periodogram analysis of seasons with sufficient data coverage yields periods between 2.51 and 3.32 days (see Fig. 7 and Table 4 ). The best period from all data between 1974 and 1994 is 2.7 days while the range in brightness is $0.8 \mathrm{mag}$. This large range is due to the low brightness of the 1982 data of Herbst et al. (1983).

HD 31738 (V1198 Ori). Balona (1987) presented 28 radial velocities in the range of +10 and $-1 \mathrm{~km} \mathrm{~s}^{-1}$ but judged these variations not as significant and listed HD 31738 as a single star. Fekel et al. (1996) found asymmetric absorption lines and concluded that the star is a double-lined spectroscopic binary. They also found a weak, sometimes even apparently absent, $\mathrm{H} \alpha$ line as well as strong Ca II $\mathrm{H}$ \& $\mathrm{K}$ emission that was confirmed by Strassmeier et al. (1990). The light variability was discovered by Hall et al. (1986) but they concluded that the comparison star was the variable. Later, Strassmeier et al. (1989a) reexamined the same data set and found that HD 31738 was the variable and not the comparison star. Their periodogram from two years of data between 1984 and 1986 gave similarly good fits at 3.70 and 4.51 days in the first season and 4.59 days in the second. No orbital period is known to date. Cutispoto (1995) observed HD 31738 in December 1989 and found the star to be constant to within 0.005 mag.

We have acquired photometry of HD 31738 since 1993 covering three full observing seasons (Fig. 8, upper panel). Combining these data with previous Vanderbilt APT observations (Hall 1996) as well as data from sources listed in Table 4 we find a sinusoidal long-term variation with an amplitude of $\approx 0.1 \mathrm{mag}$ and possibly with a period of around 15 years. However, no clear periodic variability within an observing season is detected, in agreement with Cutispoto's (1995) observations from 1989. Thus, the 4.5-day period claimed earlier by Strassmeier et al. (1989a) remains to be verified. The lower panel in Fig. 8 shows the check-minus-comparison $V$ data (HD 32263 minus HD 31594) between 1987 and 1995 and verify the long-term constancy of the comparison star although it is not clear from our data that there are no short-term variations. Clearly, higher precision is needed to solve the puzzle of HD 31738. Our periodogram analysis from prewhitened data shows one frequency at $0.214 \mathrm{~d}^{-1}(4.667$ days), however, its resulting amplitude is barely $2-\sigma$ above the noise level and has an amplitude of just $0.015 \mathrm{mag}$. We nevertheless use this period, despite that it is likely spurious, and phase the seasonal data with it. The resulting plots are given in Fig. 32. We note that if we adopt a stellar radius of $3 R_{\odot}$ from the G5IV spectral classification, an inclination of the stellar rotation axis of $45^{\circ}$ then the measured $v \sin i$ would imply a rotation period of around 5.6 days.

HD 31993 (V1192 Ori). Fekel et al. (1986) observed the star spectroscopically and gave a value for $v \sin i$ of $31 \mathrm{~km} \mathrm{~s}^{-1}$ that prompted further attention. From an independent study Balona (1987) found the star to be constant in radial velocity and thus HD 31993 seems to be a single star. The spectral classification of K2III is originally from Bidelman \& MacConnell (1973) and has been confirmed by Fekel et al. (1986). Ultraviolet IUE data and a high lithium abundance were reported by Fekel \& Balachandran (1993). The light variability was first mentioned by Lloyd-Evans \& Koen (1987) from data taken in 1980. They also found a period of 6.78 days with a very small amplitude of just $0.03-0.05$ mag in $V$. Several seasons of photometry were analyzed by Hooten \& Hall (1990), including data from as early as 1978, but their 
period analysis allowed various photometric periods between 4.8 and 29 days.

We present data from three full seasons and part of a fourth. The long-term plot is shown in Fig. 9. Clear variability is only seen in two of these seasons but verify that the 28-day period is the correct one. The $v \sin i$ and the 28-day period interpreted as the rotation period result in a minimum radius of $17 R_{\odot}$, which is still consistent with the K2III classification if the inclination of the stellar rotation axis is close to $90^{\circ}$. Seasonal light curves are shown in Fig. 33.

HD 39576. Buckley et al. (1987) drew attention to this star when they found it to be a variable X-ray emission source with moderately strong Ca II $\mathrm{H}$ and $\mathrm{K}$ emission lines. Houk (1982) had assigned a spectral type of G1V. Buckley et al. already noted that the star is likely a spectroscopic binary but no orbital elements are available to date. Strassmeier et al. (1992) discovered the light variability from data taken at ESO in 1992 and showed that the star is a very small amplitude variable with an amplitude of $0.036 \mathrm{mag}$ in Strömgren $y$ and a preliminary period of 2.7 days. Their $v \sin i$ measure of $20 \pm 2 \mathrm{~km} \mathrm{~s}^{-1}$ from a high-resolution spectrum superseded the $65 \mathrm{~km} \mathrm{~s}^{-1}$ value derived by Buckley et al. (1987) and agrees with the spectral classification of Houk (1982).

We have three full seasons of photometry and part of a fourth (Fig. 10). Periodogram analysis of each individual season gives the best period at around 2.27 days (Table 4). This is similar but still different by almost $20 \%$ to the period from the 1992 ESO data but supersedes that value. Interpreting it as the rotation period and using the $v \sin i$ of Strassmeier et al. (1992) we find a minimum radius of $0.90 R_{\odot}$, in agreement with the G1V classification of Houck. The seasonal plots are shown in Fig. 34 .

HD 81410 (IL Hya). Eggen (1973) discovered the light variability of HD 81410 and Raveendran et al. (1981, 1992) published the first orbital solution for this single-lined spectroscopic binary. Balona (1987) confirmed that finding using radial-velocity data from 1979 and 1980. Recently, Weber (1996) obtained a Doppler image of HD 81410 from KPNO spectra from 1994 showing the star with a weak polar spot. He also redetermined the spectroscopic orbit with a slightly revised period of $12.9051 \pm 0.00012$ days but in good agreement with Balona's orbit.

We have four seasons of new data of HD 81410, which brings the total baseline in time to 26 years of $V$ data (Fig. 11). The long-term average photometric period is 12.786 days and is very close to the orbital period. Figure 11 shows a more or less continuous increase in brightness since the discovery of its light variability until 1988.0 and a stagnation thereafter. The maximum $V$ amplitude from the rotationally modulated part is $\approx 0.4$ mag from 1971, but a more typical and more recent value is $0.2 \mathrm{mag}$ (e.g. between 1990-1996). The long-term variation's amplitude of the average brightness is $\approx 0.4 \mathrm{mag}$ between 1971 and 1989. The seasonal plots from our new data are shown in Fig. 35.

HD 82443. In a recent paper Henry et al. (1995a) summarized the observational history of this single K0V star and we refer the reader to this paper. They were also the first to unambiguously determine (and publish) the photometric rotation period of $5.43 \pm 0.03$ days. However, we point out that the photospheric light-curve variability was already known at the time of Henry et al.'s APT observations and had been discovered by Guinan \& McCook (1991). Just recently, Messina \& Guinan (1996) presented $U B V$ photometry of HD 82443 with the Phoenix-10 APT from February to May 1989. They found a photometric period of $5.400 \pm 0.012$ from 67 nights of observations.

Our new data are from 1994 and 1996 (Fig. 12) and braket the data of Henry et al. (1995a) from 1994/95. Differential measures were converted to absolute values by using the $V$ magnitude of our comparison star of $6.978 \pm$ $0.005 \mathrm{mag}$ (Rufener 1988). Its zero point is thus somewhat uncertain but should be good to, say, $0.01 \mathrm{mag}$. Together with the data of Messina \& Guinan (1996) we see a clear downward trend in the overall brightness of HD 82443 of 0.1 mag between 1989 and 1996, which likely indicates the existence of a decade-long spot cycle. The scatter in the seasonal plots in Fig. 36 indicates significant variations of the light curve shape on relatively short time scales.

HD 82558 (LQ Hya). LQ Hydrae is a rapidly rotating, single K2 dwarf star that probably just arrived on the zero-age main sequence (Fekel et al. 1986), while Vilhu et al. (1991) even suggested it to be a pre-main sequence object. Its light variability was discovered independently by Eggen (1984) and Fekel et al. (1986) from data taken in late 1982. A first Doppler map was presented by Strassmeier et al. (1993b) that showed a high-latitude spot straddling the rotational pole but not a polar spot as seen on several evolved RS CVn binaries and was confirmed by Saar et al. (1994) who presented further maps for 1991 and 1993.

We present four new seasons of photometric data and have now 14 years of continuous $V$ data. Figure 13 shows the long-term behavior of LQ Hya and possibly detects a sinusoidal light variation with a period of around 7 years. The seasonal light and color curves in Fig. 37 indicate a changing light curve shape with $V$ amplitudes between $0.03 \mathrm{mag}$ in $1995 / 96$ and $0.15 \mathrm{mag}$ in $1994 / 95$.

HD 106225 (HU Vir). Bidelman (1981) and Fekel et al. $(1984,1986)$ were the first who drew attention to this star and Fekel et al. also discovered its light and spectrum variability. Strassmeier (1994) presented an extensive spectroscopic and photometric study and proposed a single, large coronal loop to explain their photospheric and chromospheric Doppler images as well as the complex $\mathrm{H} \alpha$ line-profile variations. Although follow-up observations with ROSAT did not result in the proposed rotational modulation of the X-ray flux but, instead, discovered a huge X-ray flare lasting for about two days (Endl 
et al. 1995). Hudec \& Stepan (1995) used archival photographic plates to search whether HD 106225 is the optical counterpart of the luminous gamma-ray source GRB 930103. However, they did not find any variations exceeding their detection limit of $0.3 \mathrm{mag}$ within a monitoring interval of 180 hours nor 0.8 mag within an interval of 420 hours, respectively.

We have six full seasons of new photometry for HD 106225 bringing the total time coverage to 14 years (Fig. 14). Most notable in Fig. 14 is the continuous increase of the maximum system brightness since a pronounced minimum in 1992. These variations might proceed periodically with a sawtooth-like shape. The most recent data from 1996 indicate a reverse of the light increase (see Fig. 14) and, if real, a period of around 45 years could be expected. The seasonal light curves for our data from 1990 through 1996 are plotted in Fig. 38 while the seasonal periods are plotted in the lower panel of Fig. 14. The light curve shows mostly two minima except in 1995/96 where a single but asymmetric minimum is seen, indicating the long-term presence of two spotted regions. The $V$ amplitude remains more or less the same during our time coverage and is $\approx 0.2 \mathrm{mag}$ and $0.04 \mathrm{mag}$ in $V-I$.

HD 111812 (31 Com). 31 Comae is a rapidly rotating, active, single G0 giant located in the Hertzsprung gap of the HRD and as such very similar to the FK Comae type stars (e.g. Bopp et al. 1988). Its early spectral type, however, probably prevents it from being a heavily spotted star, in agreement with its low (predicted) Rossby number (Hall 1994) but despite the existence of strong Ca II H\&K emission-line fluxes similar to the most active RS CVn's (Strassmeier et al. 1990). No photometric variations were detected so far (Strassmeier \& Hall 1988a; Lockwood et al. 1993) but Strassmeier et al. (1994c) claimed to have seen line-profile variations in high-resolution spectra of photospheric absorption lines that would indicate starspots. Lockwood et al. (1993) obtained an rms error from eight years of Strömgren data of as small as 0.0029 mag but mentioned that 31 Comae is variable on a seasonal timescale.

Three seasons of new $U B V$ data of 31 Comae from the Phoenix-10 and T7 APTs are available and are plotted together with previous data from the Phoenix-10 as well as $V$ data from the Vanderbilt/TSU APT (Hall 1988) in Fig. 15. Our periodogram analysis does not reveal any significant period. However, the range of the observed $V$ magnitudes within individual observing seasons is somewhat larger than what is expected from the Phoenix-10 data quality. Also, Fig. 15 shows a continuous downward trend of the average $V$ brightness from 4.927 mag in 1984 to $4.950 \mathrm{mag}$ in 1996 . If real, it probably indicates the existence of a long-term activity cycle but without measurable rotational modulation. We note that the $v \sin i$ of 31 Comae is $57 \pm 1.5 \mathrm{~km} \mathrm{~s}^{-1}$. A value that is too large if the star were seen pole on. Adopting an inclination of $45^{\circ}$ and a radius of $6 R_{\odot}$ we would expect a rotation period of around 4 days.

HD 112313 (IN Com). Despite extensive observational efforts it is still not fully clear what IN Comae really is (see e.g. Jasniewicz et al. 1987, 1996). Here we treat it as a G5III-IV RS CVn-type binary star but note that IN Comae is related with one of the hottest stars known, an O subdwarf with $T_{\text {eff }}>120000 \mathrm{~K}$, with which it forms together the central binary system of the planetary nebula LoTr-5 (see the CABS catalog for further references). Its light variability was discovered by Schnell \& Purgathofer (1983) and several, mostly spurious, periods were found. Two very different periodicities were published thereafter: one with 0.25 days (Kuszawska \& Mikolajewski 1993), and the other with either 1.2 or 5.9 days (one is the alias of the other, see references in Table 5). One of the latter periods is interpreted as the rotation period of the G5III-IV star. It is still unknown where the 0.25-day period - if real comes from. Neither Jasniewicz et al. (1996) nor Schnell (1996) could confirm the short-period variations despite intensive monitoring in spring 1994 and 1983, respectively. Hubl \& Strassmeier (1995) presented Doppler maps with both the 1.2 and the 5.9-day period and concluded that the longer period is the more likely one. In either case the maps do not show the big, cool polar spot typical for RS CVn stars but mostly spots at low latitudes.

In this paper we also present unpublished $B V$ photometry obtained by A. Schnell at Vienna Observatory (Schnell 1996) from 1983 through 1988. New APT photometry has been obtained for four full seasons between 1993 and 1996. Figure 16 shows all available $V$ data since the discovery of the light variability in 1983. Periodogram analysis of the entire data set shows the strongest peak at 5.9345 days. Its $1-f$ alias is also present but significantly weaker and we therefore believe that the 5.9-day period is the rotation period of IN Comae. Figure 40 shows the seasonal data phased with the long-term average photometric period.

HD 113816. Buckley et al. (1987) discovered the star to be a strong X-ray source and identified it as a chromospherically active binary star. Dadonas (1996) computed a single-lined spectroscopic orbit with a period of 23.7 days. The light variability was long suspected and recently discovered by Henry et al. (1995a) with a 23.5-day modulation, making the star a synchronous rotator. Still significant differences exist in the published $v \sin i$ values for HD 113816, from the $30 \mathrm{~km} \mathrm{~s}^{-1}$ value of Buckley et al. (1987) from relatively low-resolution spectra to $10 \mathrm{~km} \mathrm{~s}^{-1}$ in Randich et al. (1994) and $5 \pm 1 \mathrm{~km} \mathrm{~s}^{-1}$ in Henry et al. (1995a) from spectra of comparable resolution and quality than Randich et al.'s. The spectrum has been classified as K0III by Henry et al. (1995a) and as K2III-IV from multicolor photometry (Buckley et al. 1987). The resulting minimum radius depends linearly on the $v \sin i$ measure and is either 2.3 or $4.6 R_{\odot}$ whether the 5 or the $10-\mathrm{km} \mathrm{s}^{-1}$ value is adopted. The former would suggest a very low inclination 
of the stellar rotation axis of just $\approx 11^{\circ}$. Because the $V$-light curve amplitude was only 0.04 mag in 1994 (Fig. 26 in Henry et al. 1995a) such a low amplitude would be in general agreement with a low inclination.

We have four full seasons of new photometry of HD 113816 from 1993 through 1996 (Fig. 17). Periodogram analysis for each observing season gives consistent periods around 23.7 days, in good agreement with the period found by Henry et al. (1995a) from their data in 1994. The long-term light curve in Fig. 17 shows the unusually faint brightness level in that season compared to the other seasons. The average seasonal difference in $V$ was 0.075 mag compared to 1993 and 0.035 mag compared to 1995. Figure 41 presents the individual seasons together with the phase plots.

Henry et al. (1995a) mentioned that their comparison star HD 113449 exhibited long-term brightness variations of about $0.02 \mathrm{mag}$ in $V$. Our T7 check-comp data (SAO 139146 - HD 113449 in our case) from four seasons (1993-1996) gave following average differential $V$ magnitudes: $1.424 \pm 0.029,1.456 \pm 0.014,1.432 \pm 0.034$, and $1.441 \pm 0.024 \mathrm{mag}$, respectively. The four-year averages in $\Delta V, \Delta R_{\mathrm{C}}$, and $\Delta I_{\mathrm{C}}$ are $1.438 \pm 0.012,1.195 \pm 0.010$, and $1.002 \pm 0.008 \mathrm{mag}$, respectively. Their standard deviations are close to or even within the respective longterm external precision and we are thus unable to confirm whether HD 113449 is a low-lamplitude long-term variable and consequently still use the comparison star for the differential magnitudes for HD 113816.

HD 116544 (IN Vir). The light variability of this EXOSAT X-ray source was discovered by Cutispoto (1992, 1994). Tagliaferri et al. (1994) included IN Vir in their lithium study and found a weak-to-moderate Li abundance. A recent Doppler-imaging study by Strassmeier (1997) brought the following results: IN Virginis is a moderately rapid rotating $\mathrm{K} 2-3$ subgiant in a 8.2 -day binary system. No secondary star is visible in the optical spectrum. Despite its relatively low $v \sin i$ of $24.0 \mathrm{~km} \mathrm{~s}^{-1}$ and a photometric rotation period of 8.2 days it shows very strong emission lines in all five optical Ca II lines as well as in $\mathrm{H} \epsilon . \mathrm{H} \alpha$ appears as a complicated two-component line with a sharp and variable absorption feature and a blue-shifted emission component - both reminiscent of a strong, inhomogeneous stellar wind. Doppler images show a polar spot with one large appendage and a temperature difference of about $1000 \mathrm{~K}$.

Three seasons of photometric data of IN Vir have been obtained and are plotted along with Cutispoto's discovery data in Fig. 18. Periodogram analysis verifies the 8.2day period. The seasonal plots in Fig. 42 reveal significant changes from season to season.

HD 117555 (FK Com). FK Comae is the prototype of a class of rapidly-rotating single giants with the strongest, non-thermal emissions encountered within stellar objects. As can be seen from the references in Table 5 FK Comae attracts a long list of interested astronomers.
Probably the most comprehensive paper on its spot and flare activity is that by Jetsu et al. (1993) where they also discovered the light curve's "flip-flop" behavior and give an improved photometric rotation period of 2.400247 days. The BVRI photometry of Petreshock et al. (1995) has been significantly updated for this paper (Petreshock 1996) and will be published in a forthcoming paper by Petreshock and colleagues. Spectroscopic studies mainly concentrated on $\mathrm{H} \alpha$ and other chromospheric-activity indicators (e.g. Walter \& Basri 1982) but only to a smaller degree on its photospheric spectrum. This is simply due to the large rotational broadening of $160 \mathrm{~km} \mathrm{~s}^{-1}$ that makes absorption lines rather shallow and hard to measure. Nevertheless, a Doppler image was obtained by Piskunov et al. (1994) and shows cool spots mainly along the stellar equator.

We present three seasons of new data of FK Comae and plot them in Fig. 19 along with data cited in Table 5. The seasonal plots are shown in Fig. 43. Note that unpublished $U B V(R I)_{\mathrm{C}}$ data from Oláh \& Jurcsik (1996) is included in our plots. Jetsu et al. (1994) presented a collection of photometric data of FK Com from various sources up to 1990 (not separated in Fig. 19) that we also use in the analysis. (Note that the data of Huenemoerder et al. (1993) were included in the Jetsu et al. paper but not the data of Eaton (1985), and also that the data of Dorren et al. (1984) are $\mathrm{H} \alpha$ narrow-band photometry and could not be used in our long-term study.) Periodogram analysis of the whole data set from 1966 to 1996 gives a period of $2.397696 \pm 0.000039$ days (see also Fig. 1). This period is derived without any further fine tuning like the elimination of the flip-flop phase behavior discovered by Jetsu et al. (1993).

HD 129333 (EK Dra). According to Dorren \& Guinan (1994) EK Draconis is the most active "solar twin" known. Its G1-2V spectral classification, strong radio and X-ray emission (Guedel 1995), and a rotation period of 2.70 days (Dorren \& Guinan 1994) places it among a group of young stars that probably just arrived on the main sequence.

We present two full seasons of $U B V(R I)_{\mathrm{C}}$ data plus part of a third between 1994 and 1996. Previously unpublished Strömgren $y$ data from Guinan (1996) were converted to Johnson $V$ with the relation given by Olsen (1983) and are plotted along with our own data in Fig. 20. The seasonal phase plots are given in Fig. 44. There seems to be a continuous decrease of the average $V$ light level of HD 129333 between 1994 and 1996 of approximately 0.05 mag. Periodogram analysis yields an average photometric period from the three seasons of 2.605 days, that we interpret to be the rotation period of HD 129333.

BD-08 3999 (UZ Lib). This RS CVn binary has been shown to display photometric variations of up to $0.35 \mathrm{mag}$ in $V$, has the spectrum of a K0 class-III giant, and rotationally broadened lines with $v \sin i=$ $67 \mathrm{~km} \mathrm{~s}^{-1}$ (Bopp et al. 1984). Grewing et al. (1989) 
discovered a hot companion star in the ultraviolet spectrum and concluded that the system is a highly evolved binary. Recently, Strassmeier (1996) derived improved orbital elements with a period of $4.768635 \pm 0.000085$ days and zero eccentricity that, together with the photometric period of Grewing et al. (1989) of $4.73574 \pm 0.00008$ days, suggests UZ Lib to be a synchronous rotator. A Doppler image shows very high latitude spots as well as equatorial features but not a cap-like polar spot as seen on other RS CVn binaries (Strassmeier 1996).

We have four full seasons of new photometric data of UZ Librae which we plot in Fig. 21 along with data cited in Table 5. Note that the data of Hoffmann (1980) were taken only in Johnson $B$ and are thus not included in our long-term $V$ plot. UZ Lib expierences a continuous brightness increase since 1972 with a full range of 0.5 mag. Its maximum $V$ amplitude due to rotational modulation was around $0.3 \mathrm{mag}$ in 1995 when the light curve appears with a single minimum (Fig. 45). The $V-I$ color variations are always in phase with the light curve and reach amplitudes of up to $0.07 \mathrm{mag}$.

HD 195040 (AT Cap). The light variability of this single-lined spectroscopic binary was discovered by LloydEvans \& Koen (1987) with a photometric period of 23.21 days and a maximum amplitude of $0.23 \mathrm{mag}$ in $V$. Hooten \& Hall (1990) listed the star in their program but did not obtain new data. The spectral classification of K2III is originally from Bidelman \& MacConnell (1973) and was confirmed by Fekel et al. (1986) who also determined the $v \sin i$ of $24 \mathrm{~km} \mathrm{~s}^{-1}$. Balona (1987) computed orbital elements with a period of 23.206 days.

We have new photometric data of HD 195040 from three observing seasons (Fig. 22), which show the star with a double-humped light curve. Periodogram analysis yields seasonal periods of around 11.6 days, very close to $1 / 2$ the orbital period. Some of the light variations might be due to an ellipticity effect in case the orbital eccentricity is indeed different from zero. However, the light curve maximum at around phase 0.6 in Fig. 46 is fainter than the one at phase 0.1 in 1995 and in 1996 but equal if not brighter in 1994 and completely absent in 1993. This indicates that the light curve variation is governed by changing starspots and that twice the photometric period is the rotation period of HD 195040.

HD 202077 (BM Mic). Lloyd-Evans \& Koen (1987) presented extensive photometry for the observing period 1979-81 and found the star to be variable with a period of 14.6 days and a $V$ amplitude of around 0.2 mag. Strassmeier et al. (1994b) obtained additional data on 15 nights in mid 1994 and confirmed the period of LloydEvans \& Koen with $14.3 \pm 0.5$ days and a $V$ amplitude of 0.06 mag. No $B-V$ nor $U-B$ color variations were seen in this data set.

We present new photometry from the four observing seasons between 1993 and 1996 (Fig. 23). Periodograms show a clear period at 14.7 days with seasonal deviations of up to 0.6 days. The individual seasons were phased with that period and are shown in Fig. 47. The scatter in the seasonal phase plots indicates both light and color changes on relatively short timescales.

HD 216489 (IM Peg). IM Pegasi is one of the more well-observed systems in the RS CVn class and appeared already in the first RS CVn list of Hall (1976). Previous photometry is summarized in Table 5 and for earlier (photometric) references we refer the reader to the papers by Poe \& Eaton (1985) and Strassmeier et al. (1989a). Here we just note that the light curve variability was originally discovered by W. Herbst in 1971 and later presented in Percy \& Welch (1982). From a preliminary study, Dempsey et al. (1994) tentatively identified a solar-like spot cycle from 15 years of photometry. For references on visual and ultraviolet spectroscopy we refer to the most recent paper on IM Peg by Dempsey et al. (1996).

We have three full seasons of $U B V(R I)_{\mathrm{C}}$ observations as well as unpublished data from Oláh (1996) from $1991 / 92$. Figure 24 is the corresponding long-term plot covering 25 years of photometry. The $V$ amplitude due to rotational modulation reached an all-time maximum of almost $0.3 \mathrm{mag}$ in mid 1995/96 while, at the same time, IM Pegasi's brightness is at an all-time low with the faintest $V$-light minimum ever observed at around 6.1 mag (compared to a maximum value of $5.64 \mathrm{mag}$ in 1986). We do not see indications for a periodic long-term behavior as suggested by Dempsey et al. (1994) but long-term light variations are clearly present.

Note that our check star for the T7 observations was the semi-regular S-type giant HR Pegasi (HD $216672=$ HR 8714, e.g. Eggen 1992) and, since our comparison star is indeed constant, we present a complete light curve for HR Peg in Fig. 25.

\subsection{Data archiving and availability}

All new APT data presented in this paper is available at CDS from

http://cdsweb.u-strasbg.fr/Abstract.html

and from the AstroSERVERVIENNA under following URL (then click on "publications")

http://www.ast.univie.ac.at/kgs/StellarActivity.html

Acknowledgements. Research with automated photometry at the University of Vienna is supported by a governmental grant to the Institute for Astronomy and by the Joint Research Initiative on stellar astrophysics of the Austrian Fond zur Förderung der wissenschaftlichen Forschung (FWF). Within this initiative KGS acknowledges the support through grant S7301-AST, and grant OWP-40 from the Austrian Academy of Sciences via their East-West program. Stellar activity research at Catania Observatory is supported by the Italian Ministry 
for Scientific Research and Technology, the Italian National Council for Research ("Gruppo Nazionale di Astronomia") and the Sicilian Regional Government. GC acknowledges the hospitality at Vienna Observatory through grant S-7300-AST. Besides the cash flow, it is also a great pleasure to thank Lou Boyd and Don Epand of Fairborn Observatory for their continuous operation of and help with the T7 APT and its data reduction. Thanks also go to Mike Seeds of Franklin \& Marshall College for keeping the Phoenix-10 APT busy and to the whole TSU-Center of Excellence group for continuous collaboration. Following colleagues allowed us to use their data prior to publication: D.S. Hall, W. Barksdale, B. Skiff and H. Nations, K. Oláh and H. Jurcsik, J. Petreshock, and A. Schnell, we gratefully acknowledge their generousity. This research has greatly benefited from use of the SIMBAD data base, operated at CDS in Strasbourg, France.

\section{References}

Alekseev I.Y., 1996, Astron. Reports 40, 74

Baliunas S.L., Blair W.P., Guinan E.F., 1983, IBVS 2323

Baliunas S.L., Soon W., 1995, ApJ 450, 896

Balona L.A., 1987, SAAO Circ. 11, 1

Barksdale W.S., 1990 (private communication)

Bartolini C., Blanco C., Catalano S., et al., 1983, A\&A 117, 149

Bevington P.R., 1969, Data Reduction and Error Analysis for the Physical Sciences. McGraw-Hill, New York

Bidelman W.P., 1981, AJ 86, 553

Bidelman W.P., MacConnell D.J., 1973, AJ 78, 687

Bieging J.H., Cohen M., 1989, AJ 98, 1686

Biermann L., 1941, Mitt. Astron. Ges. 76, 283

Bohusz E., Udalski A., 1983, IBVS 2390

Bondar N.I., 1995, A\&AS 111, 259

Bond H.E., Livio M., 1990, ApJ 355, 568

Bopp B.W., 1996 (private communication)

Bopp B.W., Africano J.L., Stencel R.E., Noah P.V., Klimke A., 1983, ApJ 275, 691

Bopp B.W., Dempsey R.C., Maniak S., 1988, ApJS 68, 803

Bopp B.W., Evans D.S., 1973, MNRAS 164, 343

Bopp B.W., Goodrich B.D., Africano J.L., et al., 1984, ApJ 285,202

Bopp B.W., Saar S.H., Ambruster C., et al., 1989, ApJ 339, 1059

Bopp B.W., Fekel F.C., Aufdenberg J., Dempsey R., Dadonas V., 1993, AJ 106, 2502

Bouvier J., Bertout C., Benz W., Mayor M., 1986, A\&A 165, 110

Bouvier J., Bertout C., Bouchet P., 1988, A\&AS 75, 1

Bouvier J., Cabrit S., Fernandez M., Martin E.L., Matthews J.M., 1993, A\&AS 75, 1

Boyd L.J., Genet R.M., Hall D.S., 1984, IAPPP Commun. 15, 20

Boyd L.J., Genet R.M., Hall D.S., Busby M.R., Henry G.W., 1990, IAPPP Commun. 42, 44

Breger M., 1990, Commun. Astroseismology 20, 1

Buckley D.A.H., Tuohy I.R., Remillard R.A., Bradt H.V., Schwartz D.A., 1987, ApJ 315, 273

Catalano S., Rodonó M., 1967, Mem. S.A.It. 38, 345

Cellino A., Scaltriti F., Busso M., 1986, Ap\&SS 121, 265
Choi H.-J., Soon W., Donahue R.A., Baliunas S.L., Henry G.W., 1995, PASP 107, 744

Chugainov P.F., 1966, IBVS 172

Chugainov P.F., 1976, Izv. Krym. Astrofiz. Obs. 55, 85

Chugainov P.F., Lovkaya M.N., Zajtseva G.V., 1991, IBVS 3680

Cohen M., Schwartz R.D., 1976, MNRAS 174, 137

Collier-Cameron A., 1987, SAAO Circ. 11, 57

Cutispoto G., 1990, A\&AS 84, 397

Cutispoto G., 1991, A\&AS 89, 435

Cutispoto G., 1993, A\&AS 102, 655

Cutispoto G., 1995, A\&AS 111, 507

Cutispoto G., 1996, A\&AS 119, 281

Cutispoto G., Giommi P., Pallavicini R., et al., 1992, in Giampapa M.S. and Bookbinder J.A. (eds.) Cool Stars, Stellar Systems, and the Sun, PASPC 26, p. 119

Cutispoto G., Pallavicini R., Rodonó M., Tagliaferri G., 1994, in Caillault J.P. (ed.) Cool Stars, Stellar Systems, and the Sun, PASPC 64, p. 687

Cutispoto G., Tagliaferri G., Pallavicini R., Pasquini L., Rodonó M., 1996, A\&AS 115, 41

Dadonas V., 1995 (private communication)

Dempsey R.C., Bopp B.W., Henry G.W., Hall D.S., 1994, in Caillault J.P. (ed.) Cool Stars, Stellar Systems, and the Sun, PASPC 64, p. 393

Dempsey R.C., Neff J.E., O’Neal D., Oláh K., 1996, AJ 111, 1356

Dorren J.D., Guinan E.F., 1994, ApJ 428, 805

Dorren J.D., Guinan E.F., McCook G.P., 1984, PASP 96, 250

Eaton J.A., 1985, IAU Comm. 27 Archive of Unpublished Photometry, Data File Number 147

Eaton J.A., Poe C.H., 1986, IBVS 2846

Eaton J.A., Hall D. S., Henry G.W., et al., 1983, Ap\&SS 89, 53

Eggen O.J., 1973, PASP 85, 42

Eggen O.J., 1984, AJ 89, 1358

Eggen O.J., 1992, AJ 104, 275

Endl M., Strassmeier K.G., Kürster M., 1995, in Strassmeier K.G. (ed.), Poster Proceedings: Stellar Surface Structure, IAU Symp. 176, Univ. Vienna, p. 203

Fekel F.C., Balachandran S., 1993, ApJ 403, 708

Fekel F.C., Bopp B.W., Africano J.L., et al., 1986, AJ 92, 1150

Fekel F.C., Hall D.S., Henry G.W., 1984, IBVS 2543

Fekel F.C., Hall D.S., Henry G.W., Landis H.J., Renner T.R., 1982, IBVS 2110

Fekel F.C., Marschall L.A., 1991, AJ 102, 1439

Fekel F.C., Moffett T.J., Henry G.W., 1986, ApJS 60, 551

Fekel F.C., Quigley R., Gilles K., Africano J.L., 1987, AJ 94, 726

Gahm G., Gullbring E., Fischerstrom C., Lindroos K.P., Loden K., 1993, A\&AS 100, 371

Giampapa M.S., Basri G.S., Johns C.M., Imhoff C.L., 1993, ApJS 89, 321

Grankin K.N., 1994, IBVS 4042

Gray D.F., Baliunas S.L., Lockwood G.W., Skiff B.A., 1996, ApJ 456, 365

Gray D.F., Baliunas S.L., Lockwood G.W., Skiff B.A., 1992, ApJ 400, 681

Grewing M., Bianchi L., Garrido R., 1989, A\&A 223, 172

Griffin R.F., Gunn J.E., Zimmerman B.A., Griffin R.E.M., 1985, AJ 90, 609 
Güdel M., Schmitt J.H.M.M., Benz A.O., Elias II N.M., 1995, A\&A 301, 201

Guinan E.F., 1996 (private communication)

Guinan E.F., McCook G.P., 1991, BAAS 23, 874

Hall D.S., 1972, PASP 84, 323

Hall D.S., 1976, in Fitch W.S. (ed.) Multiple Periodic Variable Stars, IAU Colloq. 29. Reidel, Dordrecht, p. 287

Hall D.S., 1988 (private commununication)

Hall D.S., 1994, in D'Antona F. et al. (eds.) Evolutionary Links in the Zoo of Interactive Binaries, Mem. S.A.It. 65, p. 73

Hall D.S., 1995, in Henry G.W. and Eaton J.A. (eds.) Robotic telescopes: current capabilities, present developments, and future prospects for automated astronomy, PASPC 79, p. 65

Hall D.S., 1996 (private communication)

Hall D.S., Henry G.W., 1992, IBVS 3693

Hall D.S., Kirkpatrick J.D., Seufert E.R., 1986, IAPPP Commun. 25, 32

Hall D.S., Kirkpatrick J.D., Seufert E.R., Henry G.W., 1986, IAPPP Commun. 25, 43

Hall D.S., Osborn S.A.G., Seufert E.R., Boyd L.J., Genet R.M., Fried R.E., 1987, AJ 94, 723

Hampton M., Henry G.W., Eaton J.A., Nolthenius R.A., Hall D.S., 1996, PASP 108, 68

Hartmann L., Bopp B.W., Dussault M., Noah P.V., Klimke A., 1981, ApJ 249, 662

Hatzes A.P., 1995, ApJ 451, 784

Hatzes A.P., Vogt S.S., 1992, MNRAS 258, 387

Heckert P.A., 1992, IBVS 3796

Heckert P.A., 1993a, IBVS 3954

Heckert P.A., 1993b, IBVS 3953

Heckert P.A., Hickman M.A., 1991, IBVS 3570

Heckert P.A., Maloney G.V., 1992, IBVS 3700

Heckert P.A., Maloney G.V., Stewart M., 1992, IBVS 3713

Henry G.W., 1995, in Henry G.W. and Eaton J.A. (eds.) Robotic telescopes: current capabilities, present developments, and future prospects for automated astronomy, PASPC 79, p. 44

Henry G.W., Eaton J.A., 1995, Robotic telescopes: current capabilities, present developments, and future prospects for automated astronomy, PASPC 79

Henry G.W., Eaton J.E., Hamer J., Hall D.S., 1995b, ApJS 97, 513

Henry G.W., Fekel F.C., Hall D.S., 1995a, AJ 110, 2926

Herbig G.H., Goodrich R.W., 1986, ApJ 309, 294

Herbst W., 1973, A\&A 26, 137

Herbst W., 1989, AJ 98, 2268

Herbst W., Booth J.F., Koret D.L., et al., 1987, AJ 94, 137

Herbst W., Herbst D.K., Grossman E.J., Weinstein D., 1994, AJ 108, 1906

Herbst W., Holtzman J.A., Klasky R.S., 1983, AJ 88, 1648

Herbst W., Koret D.L., 1988, AJ 96, 1949

Hoffmann M., 1980, IBVS 1878

Hoffmeister C., 1965, Veröff. Sternwarte Sonneberg 6, 97

Holtzman J.A., Herbst W., Booth J., 1986, AJ 92, 1387

Holtzman J.A., Nations H.L., 1984, AJ 89, 391

Hooten J.T., Hall D.S., 1990, ApJS 74, 225

Hooten J.T., Strassmeier K.G., Hall D.S., et al., 1989, Ap\&SS 155,45

Houk N., 1982, Michigan Catalog of 2-D Spectral Types, Ann Arbor, Vol. 3
Hubl B., Strassmeier K.G., 1995, in Strassmeier K.G. (ed.) Poster Proceedings: Stellar Surface Structure, IAU Symp. 176, Univ. Vienna, p. 96

Huenmoerder D.P., Ramsey L.W., Buzasi D.L., Nations H.L., 1993, ApJ 404, 316

Hudec R., Stepan P., 1995, A\&A 302, 407

Huovelin J., Piirola V., Vilhu O., Efimov Y.S., Shakhovskoy N.M, 1987, A\&A 176, 83

Jasniewicz G., Acker A., Mauron N., Duquennoy A., Cuypers J., 1994, A\&A 286, 211

Jasniewicz G., Duquennoy A., Acker A., 1987, A\&A 180, 145

Jasniewicz G., Thévenin F., Monier R., Skiff B.A., 1996, A\&A 307,200

Jetsu L., 1993, A\&A 276, 345

Jetsu L., Pelt J., Tuominen I., 1993, A\&A 278, 449

Jetsu L., Huovelin J., Tuominen I., et al., 1990, A\&A 236, 423

Jetsu L., Tuominen I., Antov A., et al., 1994, A\&AS 103, 183

Johns C.M., Basri G.S., 1995, ApJ 449, 341

Joncour I., Bertout C., Ménard F., 1994, A\&A 285, L25

Jurcsik H., 1996 (private communication)

Kollath Z., 1990, MUFRAN Users Manual, Occ. Techn. Notes at Konkoly Observatory 1

Kron G.E., 1947, PASP 59, 261

Kron G.E., 1952, ApJ 115, 301

Kuczawska E., Mikolajewski M., 1993, Acta Astron. 43, 445

Lloyd-Evans T., Koen M.C.J., 1987, SAAO Circ. 11, 21

Lockwood G.W., Skiff B.A., Thompson D.T., 1993, in Butler C.J. and Elliott I. (eds.), Stellar Photometry - Current Techniques and Future Developments, IAU Colloq. 136. Cambridge Univ. Press, p. 99

Malasan H.L., Yamasakai A., Kondo M., 1991, AJ 101, 2131

Manfroid J., Sterken C., Bruch A., et al., 1991, A\&AS 87, 48

Manfroid J., Sterken C., Cunow B., et al., 1995, A\&AS 109, 329

Marchev D.V., Kjurkchieva D.P., 1993, IBVS 3940

Mekkaden M.V., Sinachopoulos D., 1988, IBVS 3228

Messina S., Guinan E.F., 1996, IBVS 4286

Morris S.L., Milone E.F., 1983, PASP 95, 376

Nations H.L., 1989, BAAS 21, 1148

Nations H.L., Holtzman J.A., 1987, BAAS 19, 955

Nolthenius R., 1991, IBVS 3589

Noskova R.I., 1989, Sov. Astron. Lett. 15, 149

Oja T., 1985, A\&AS 61, 331

Oláh K., 1996 (private communication)

Oláh K., Jurcsik H., 1996 (private communication)

Oláh K., Bartus J., Kovari Z., et al., 1997, A\&A 321, 811

Oláh K., Hall D.S., Henry G.W., 1991, A\&A 251, 531

Oláh K., Pettersen B.R., 1991, A\&A 242, 443

Olsen E.H., 1980, A\&AS 39, 205

Olsen E.H., 1983, A\&AS 54, 55

Pallavicini R., Cutispoto G., Randich S., Gratton R., 1993, A\&A 267, 145

Parenago P., 1931, Variable Stars 3, 99

Paunzen E., Strassmeier K.G., 1996, IBVS 4294

Percy J.R., Welch D.L., 1982, JRASC 76, 185

Petreshock J., 1996 (private communication)

Petreshock J., Wolk S., Adams N., Walter F., 1995, BAAS 27, 815

Petrov P.P., Gullbring E., Gahm G., et al., 1995, in Strassmeier K.G. (ed.) Poster Proceedings: Stellar Surface Structure, IAU Symp. 176, Univ. Vienna, p. 217 
Petrov P.P., Shcherbakov V.A., Berdyugina S.V., et al., 1994, A\&AS 107, 9

Phillips M.J., Hartmann L., 1978, ApJ 227, 182

Piskunov N.E., Huenemoerder D.P., Saar S.H., 1994, in Caillault J.P. (ed.) Eight Cambridge Workshop, Cool Stars, Stellar Systems, and the Sun, PASPC 64, p. 658

Poe C.H., Eaton J.A., 1985, ApJ 289, 644

Randich S., Giampapa M., Pallavicini R., 1994, A\&A 283, 893

Raveendran A.V., Mekkaden M.V., Mohin S., 1981, IBVS 1975

Raveendran A.V., Mekkaden M.V., Mohin S., 1982, MNRAS 199, 707

Rice J.B., Strassmeier K.G., 1996, A\&A 316, 164

Rodonó M., Cutispoto G., 1992, A\&AS 95, 55

Rodonó M., Cutispoto G., 1994, in D'Antona F. et al. (eds.) Evolutionary Links in the Zoo of Interactive Binaries, Mem. S.A.It. 65 , p. 83

Rodonó M., Lanza A.F., Catalano S., 1995, A\&A 301, 75

Romano G., 1975, Mem. S.A.It. 46, 81

Rössiger S., 1981, Mitt. Veränd. Sterne 9, 35

Rucinski S.M., 1981a, A\&A 104, 260

Rucinski S.M., 1981b, Acta Astron. 31, 363

Rufener F., 1988, Catalog of Stars measured in the Geneva Observatory Photometric System (fourth edition) Observatoire de Genève, Sauverny

Rydgren A.E., Schmelz J.T., Zak D.S., Vrba F.J., 1984, Publ. U.S. Naval Obs. 25, part 1

Rydgren A.E., Strom S.E., Strom K.M., 1976, ApJS 30, 307

Rydgren A.E., Vrba F.J., 1983, ApJ 267, 191

Saar S.H., Piskunov N.E., Tuominen I., 1994, in Caillault J.P. (ed.) Eight Cambridge Workshop, Cool Stars, Stellar Systems, and the Sun, PASPC 64, p. 661

Scheible M.P., Guinan E.F., 1994, IBVS 4110

Schnell A., 1996 (private communication)

Schnell A., Purgathofer A., 1983, A\&A 127, L5

Schrijver C.J., Zwaan C., 1991, A\&A 251, 183

Seeds M.A., 1995, in Henry G.W. and J.A. Eaton (eds.) Robotic telescopes: current capabilities, present developments, and future prospects for automated astronomy, PASPC 79, p. 11

Skiff B., Nations H.L., 1991 (private communication)

Spurr A.J., Hoff D.B., 1987, IBVS 3028
Sterken C., 1983, The Messenger 33, 10

Sterken C., Manfroid J., Anton K., et al., 1993, A\&AS 102, 79

Sterken C., Manfroid J., Beele D., et al., 1995, A\&AS 113, 31

Strassmeier K.G., 1990, ApJ 348, 682

Strassmeier K.G., 1994, A\&A 281, 395

Strassmeier K.G., 1996, A\&A 314, 558

Strassmeier K.G., 1997, A\&A 319, 535

Strassmeier K.G., Bopp B.W., 1992, A\&A 259, 183

Strassmeier K.G., Boyd L.J., Epand D.H., Granzer Th., 1997, PASP 109, June

Strassmeier K.G., Fekel F.C., Bopp B.W., Dempsey R.C., Henry G.W., 1990, ApJS 72, 191

Strassmeier K.G., Hall D.S., 1988a, ApJS 67, 439

Strassmeier K.G., Hall D.S., 1988b, ApJS 67, 453

Strassmeier K.G., Hall D.S., Boyd L.J., Genet R.M., 1989a, ApJS 69, 141

Strassmeier K.G., Hall D.S., Eaton J.E., et al., 1988, A\&A 192, 135

Strassmeier K.G., Hall D.S., Fekel F.C., Scheck M., 1993a, A\&AS 100, 173 (CABS)

Strassmeier K.G., Hall D.S., Henry G.W., 1994a, A\&A 282, 535

Strassmeier K.G., Maitzen H.M., Pranka M., 1992, IBVS 3735

Strassmeier K.G., Neff J.E., Rodonó M., 1989b, in Hayes D.S. and Genet R.M. (eds.) Remote Access Automatic Telescopes. Fairborn Press, Fairborn, p. 197

Strassmeier K.G., Oláh K., 1992, A\&A 259, 595

Strassmeier K.G., Paunzen E., North P., 1994b, IBVS 4066

Strassmeier K.G., Rice J.B., Wehlau W.H., Hill G.M., Matthews J.M., 1993b, A\&A 268, 671

Strassmeier K.G., Rice J.B., Wehlau W.H., et al., 1991, A\&A 247,130

Strassmeier K.G., Washüttl A., Rice J.B., 1994c, IBVS 3994

Strassmeier K.G., Welty A.D., Rice J.B., 1994d, A\&A 285, L17

Tagliaferri G., Cutispoto G., Pallavicini R., Randich S., Pasquini L., 1994, A\&A 285, 272

Vilhu O., Gustafsson B., Walter F.M., 1991, A\&A 241, 167

Vrba F.J., Herbst W., Booth J.F., 1988, AJ 96, 1032

Weber M., 1996, Diplomarbeit, Universität Wien

Walter F.M., Basri G.S., 1982, ApJ 260, 735

Wisniewski W.Z., 1973, MNRAS 161, 331 


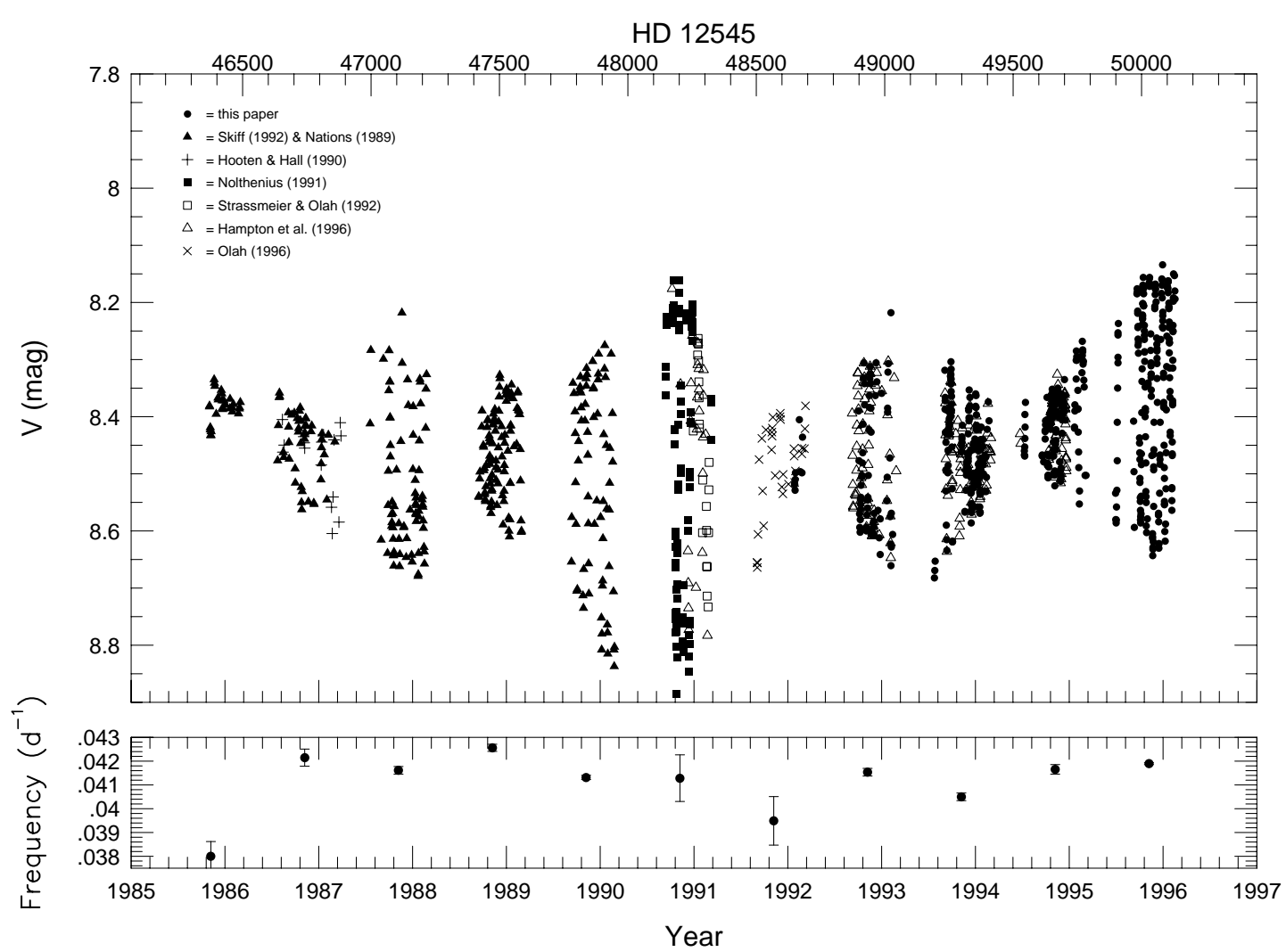

Fig. 2. HD $12545=\mathrm{XX}$ Tri

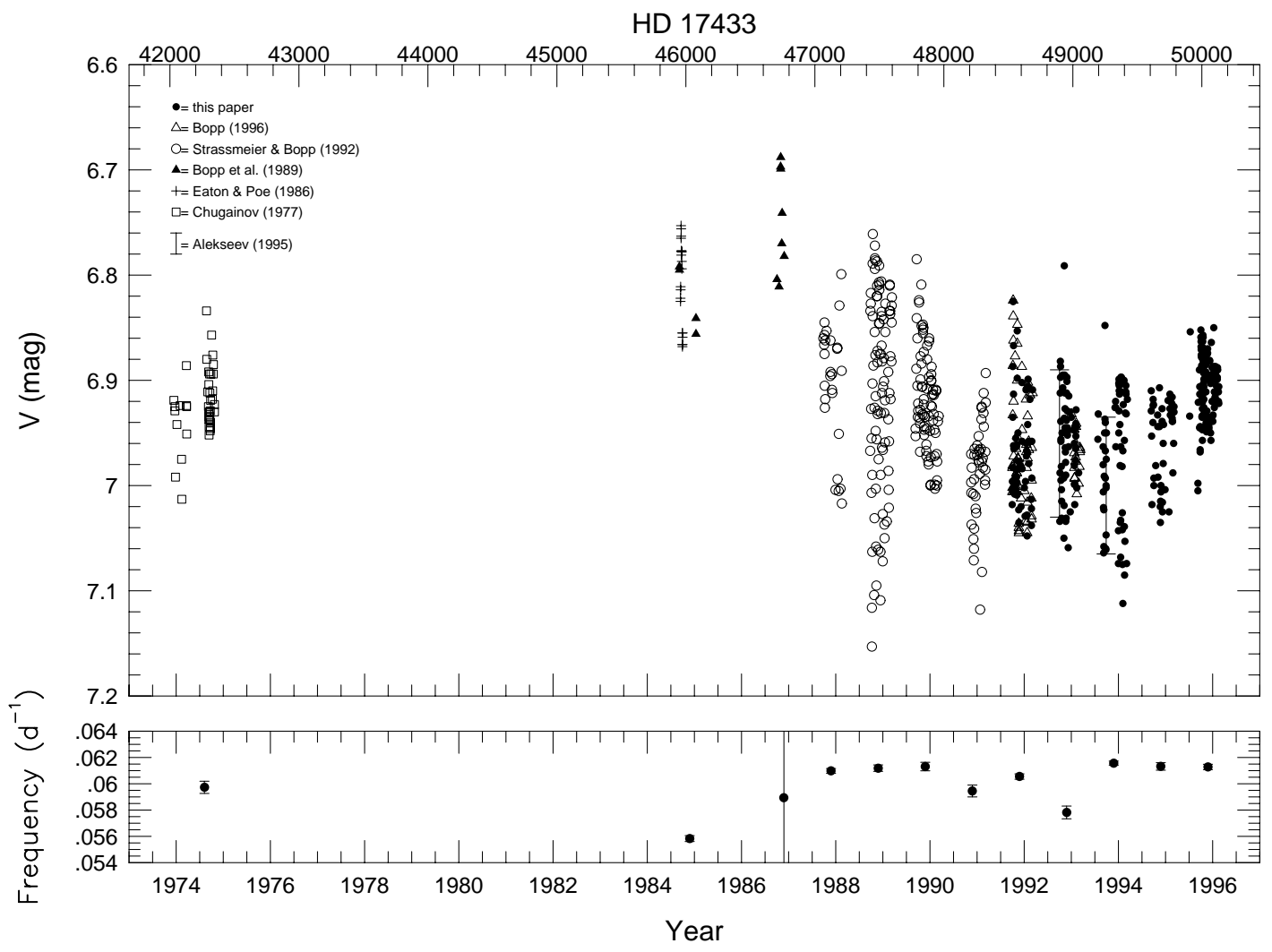

Fig. 3. HD $17433=$ VY Ari 


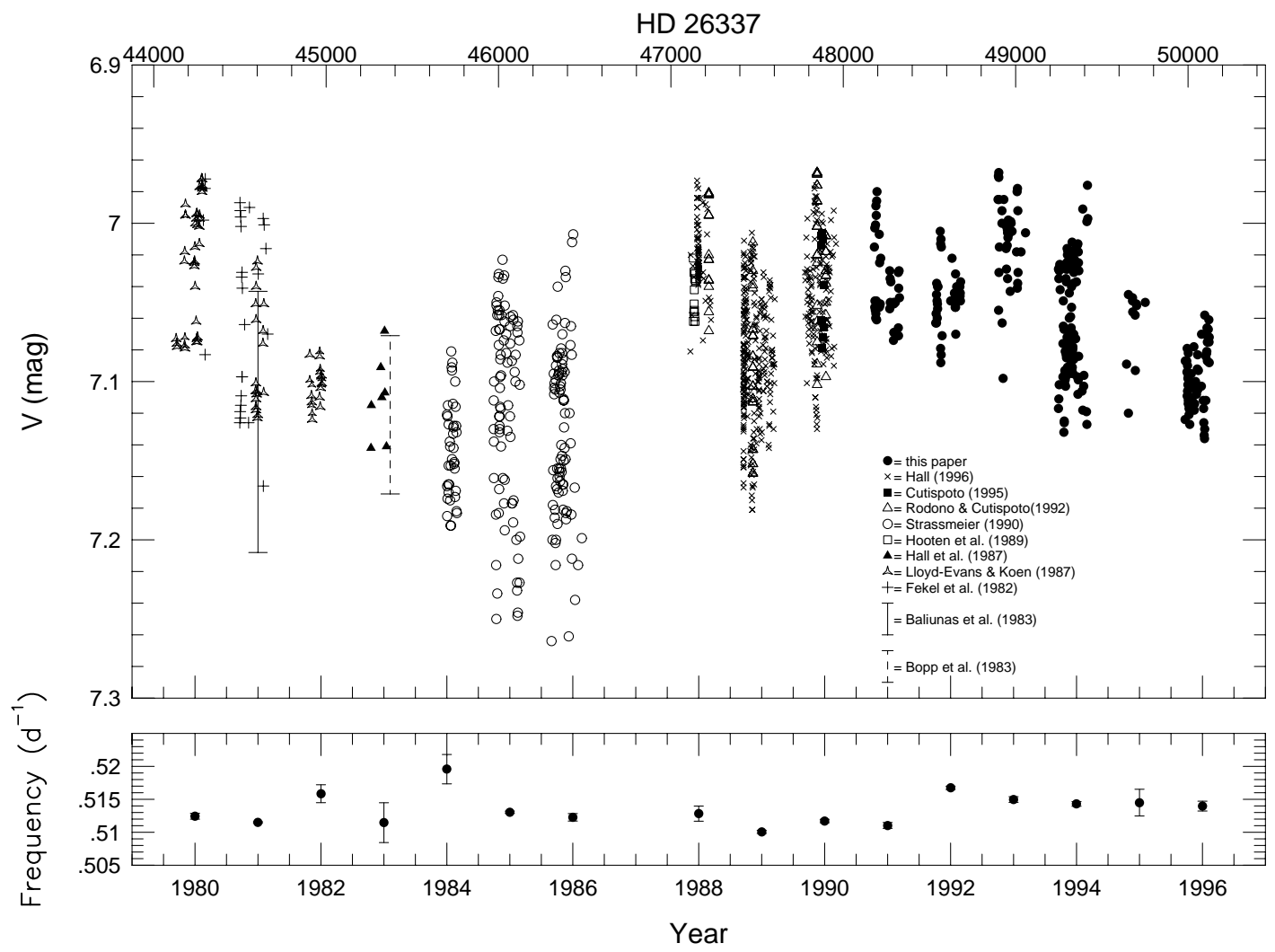

Fig. 4. HD 26337 = EI Eri

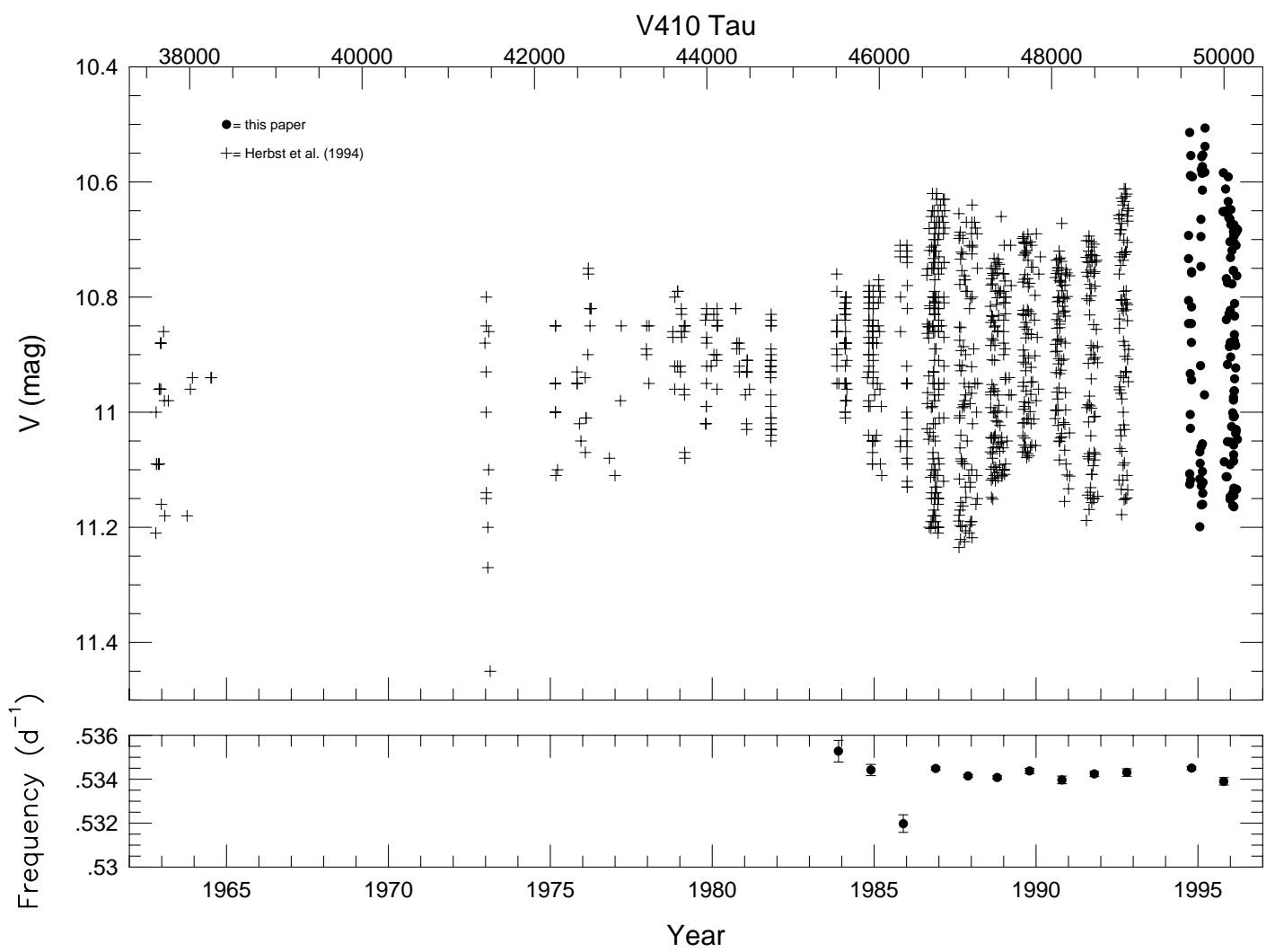

Fig. 5. V410 Tau 


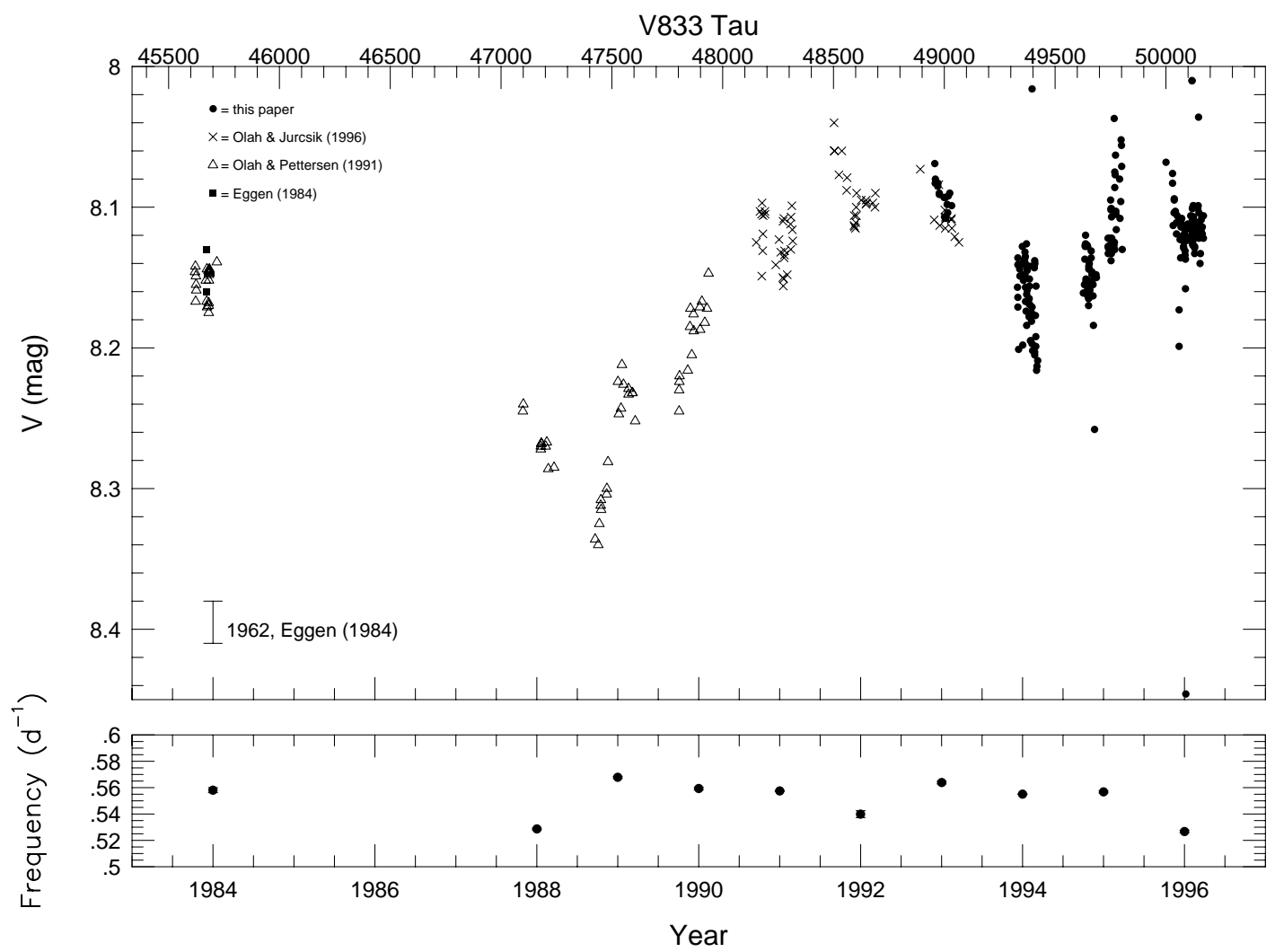

Fig. 6. HD $283750=$ V833 Tau

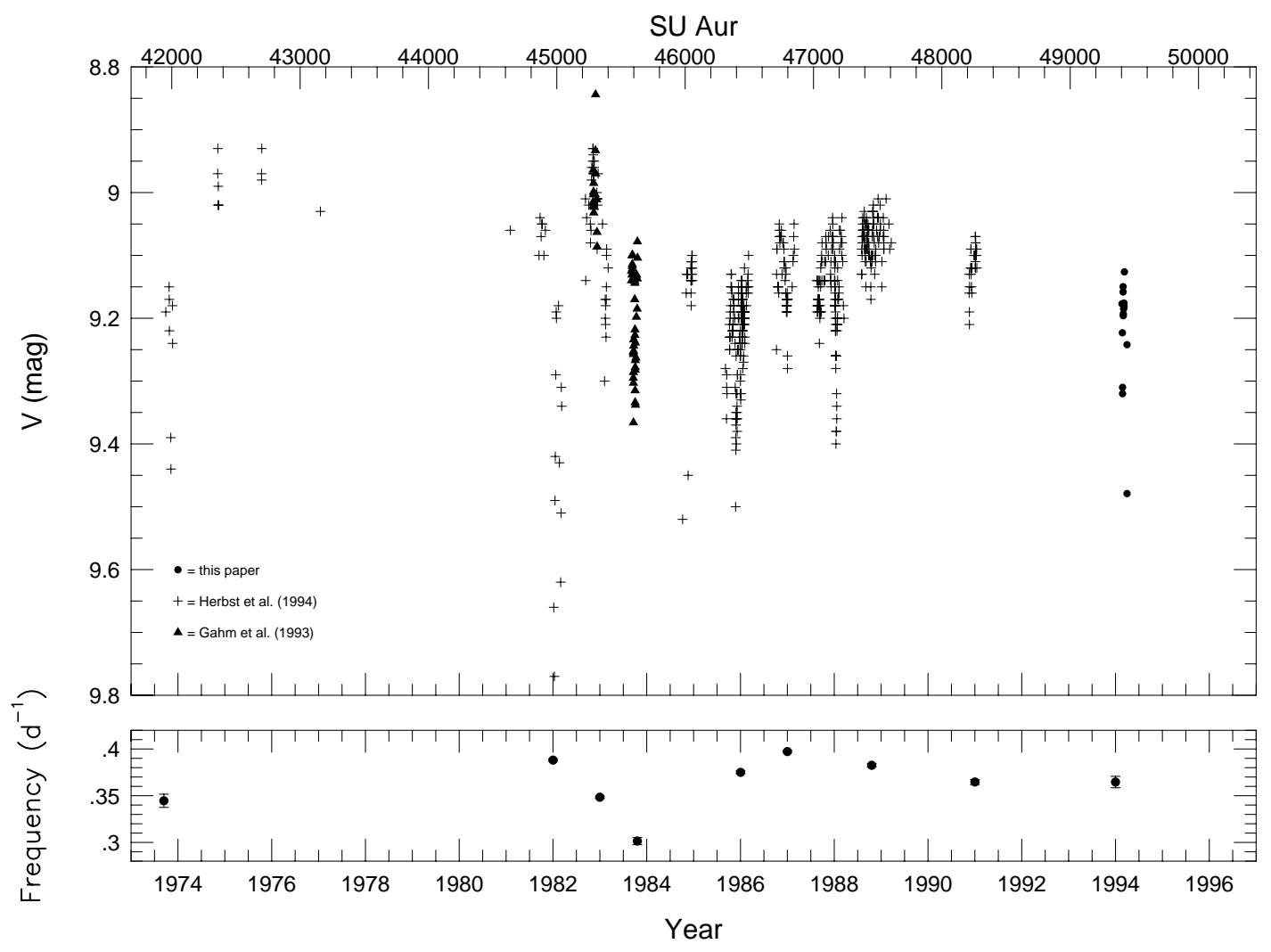

Fig. 7. SU Aur 

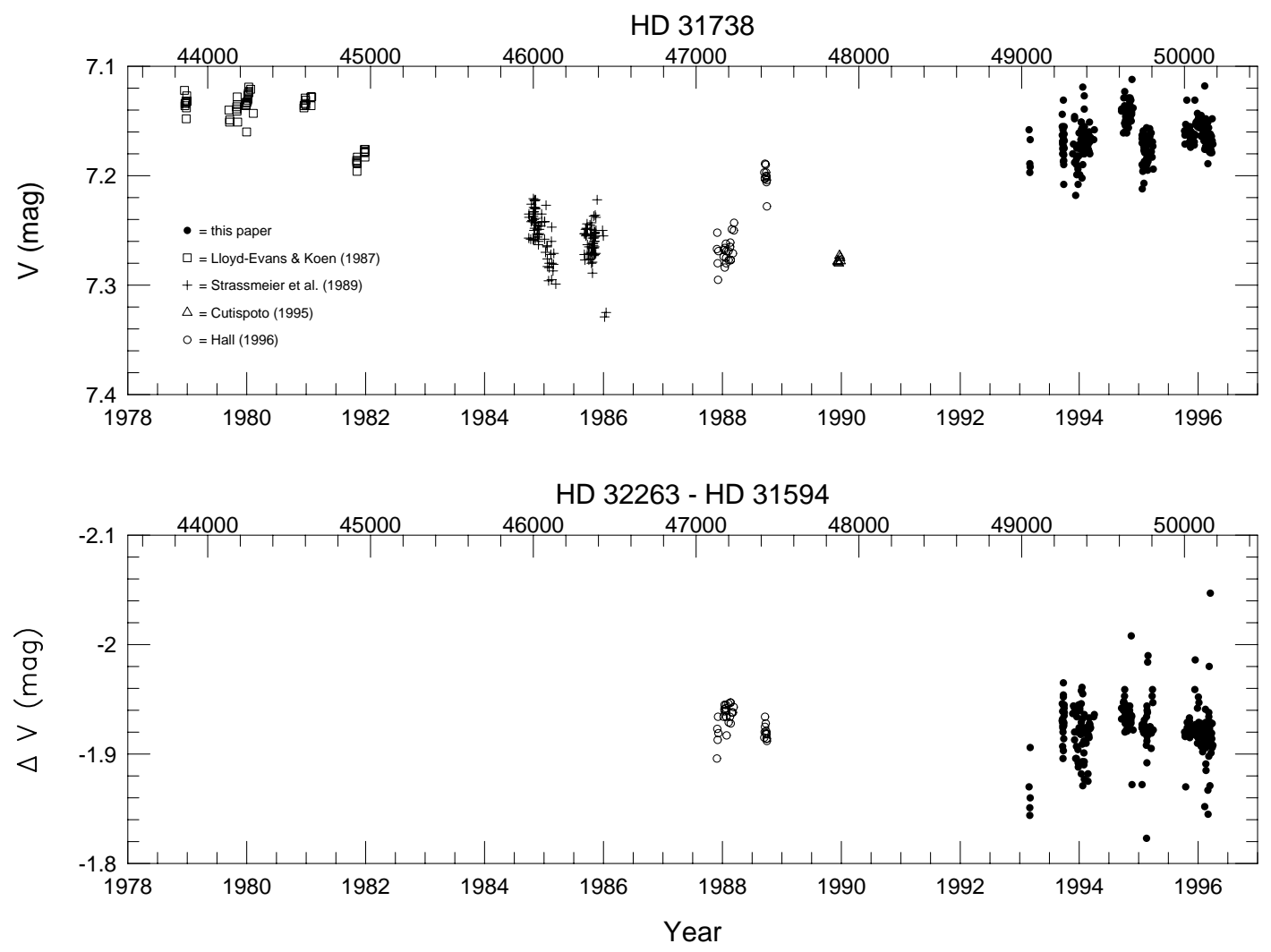

Fig. 8. HD $31738=$ V1198 Ori

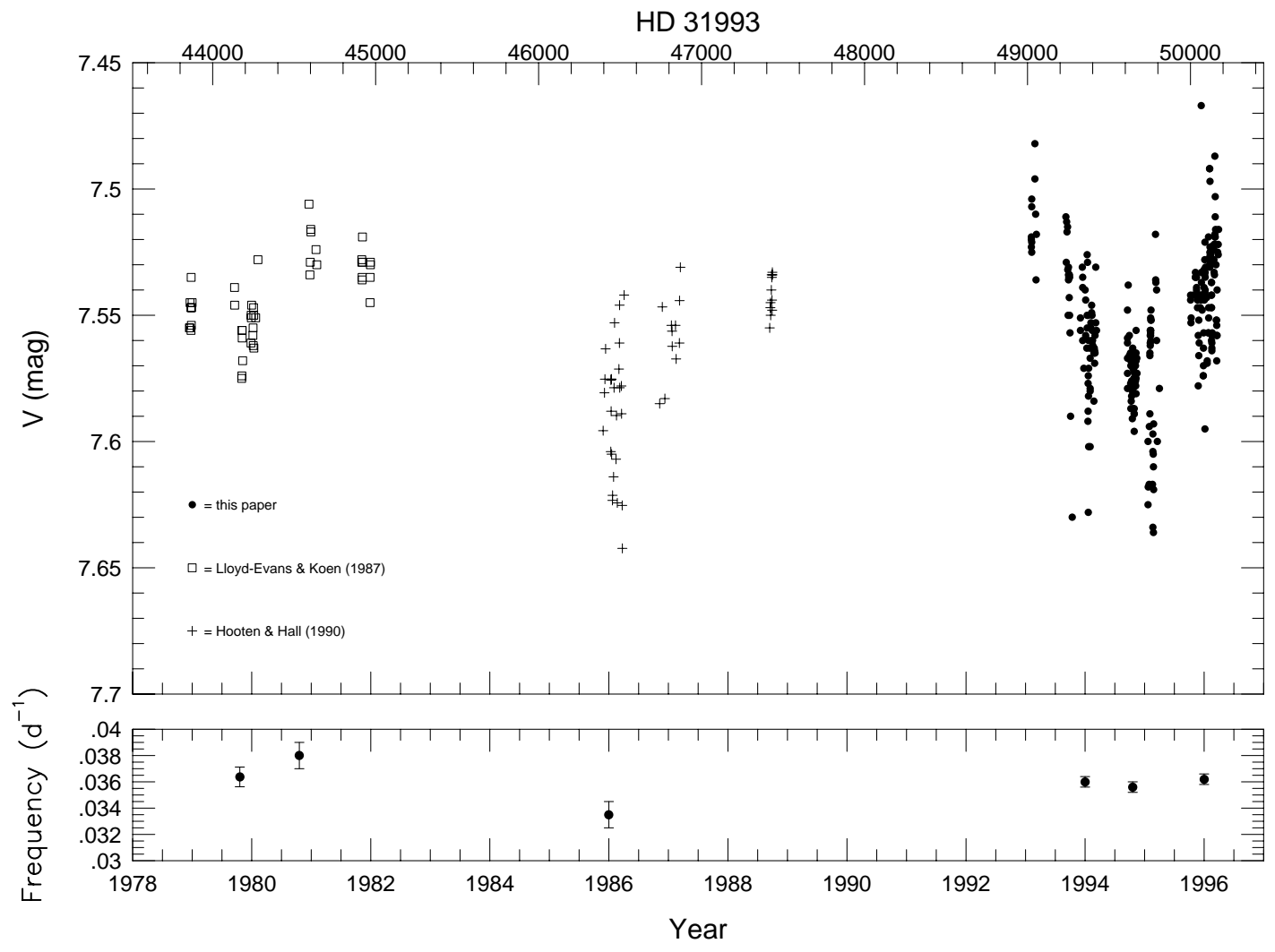

Fig. 9. HD $31993=$ V1192 Ori 


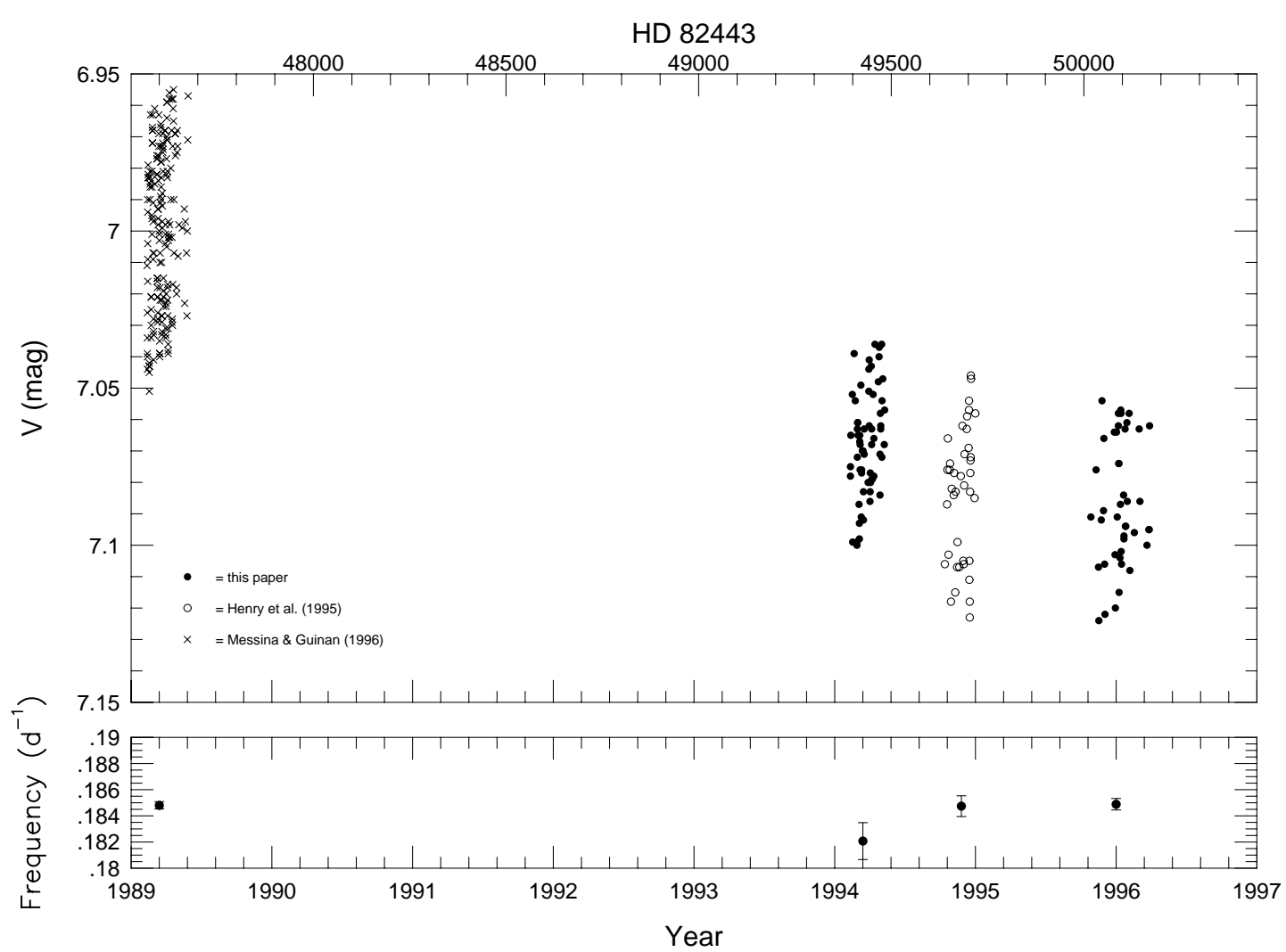

Fig. 12. HD 82443

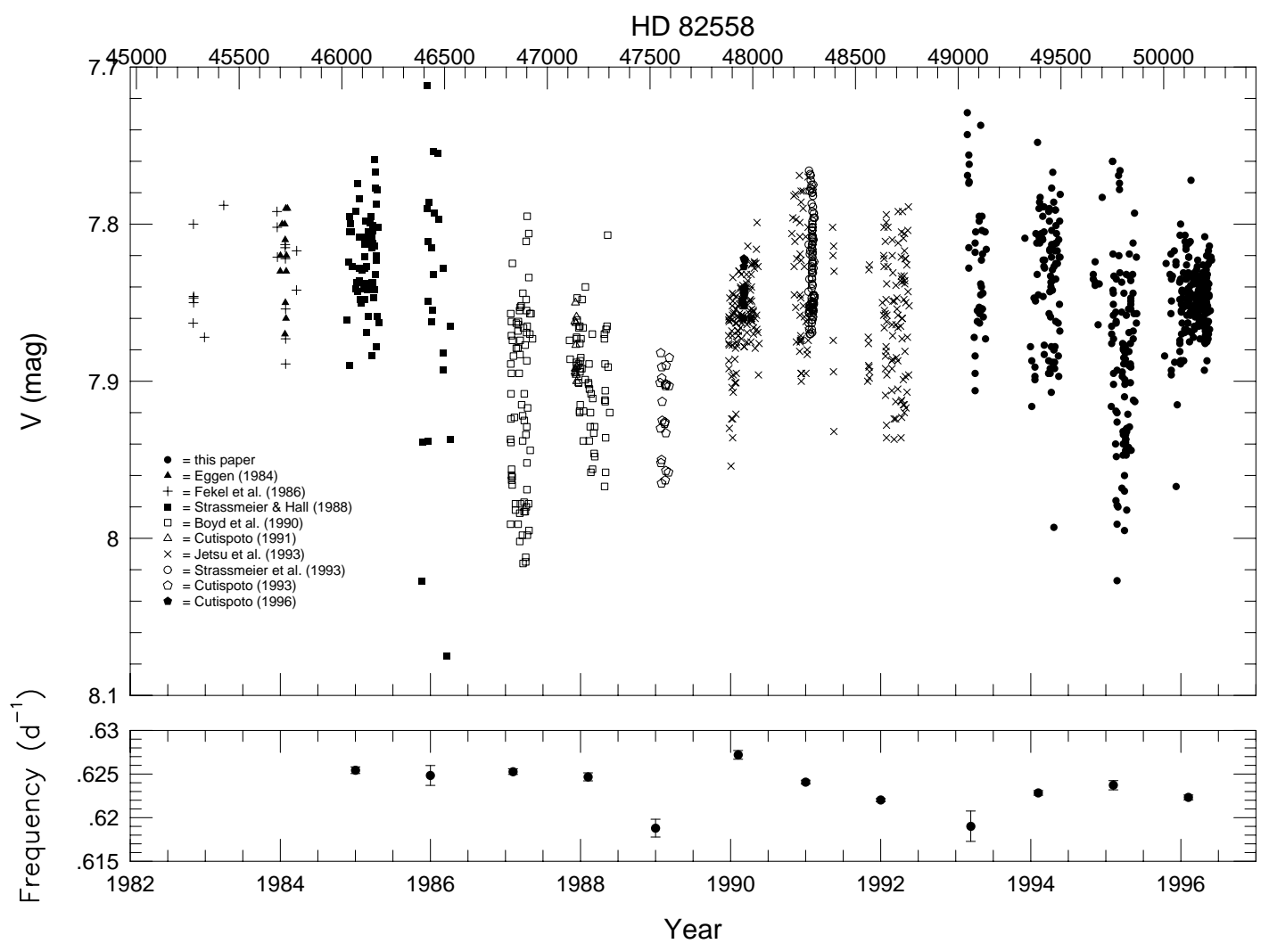

Fig. 13. HD $82558=$ LQ Hya 
HD 106225

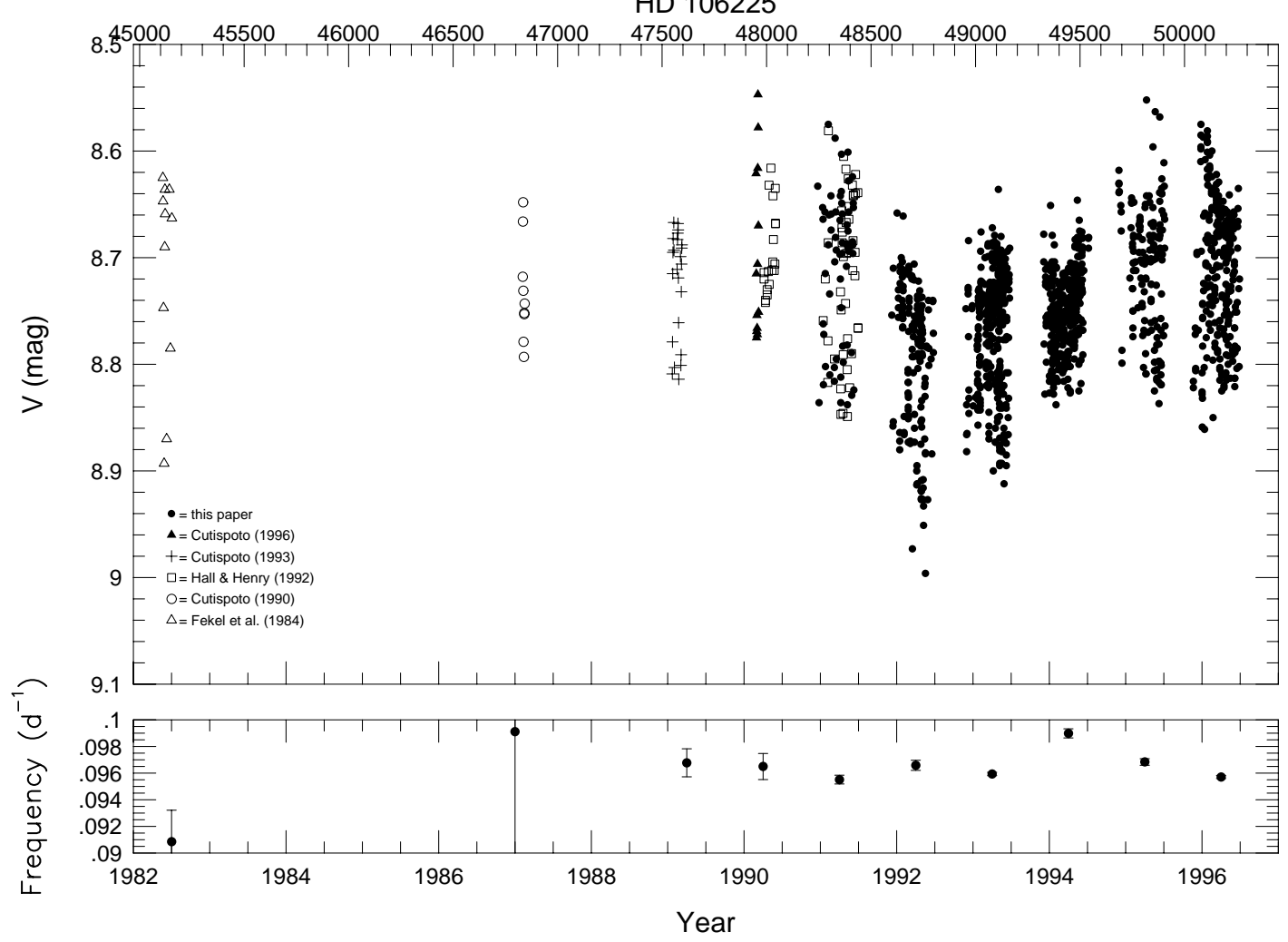

Fig. 14. HD $106225=$ HU Vir

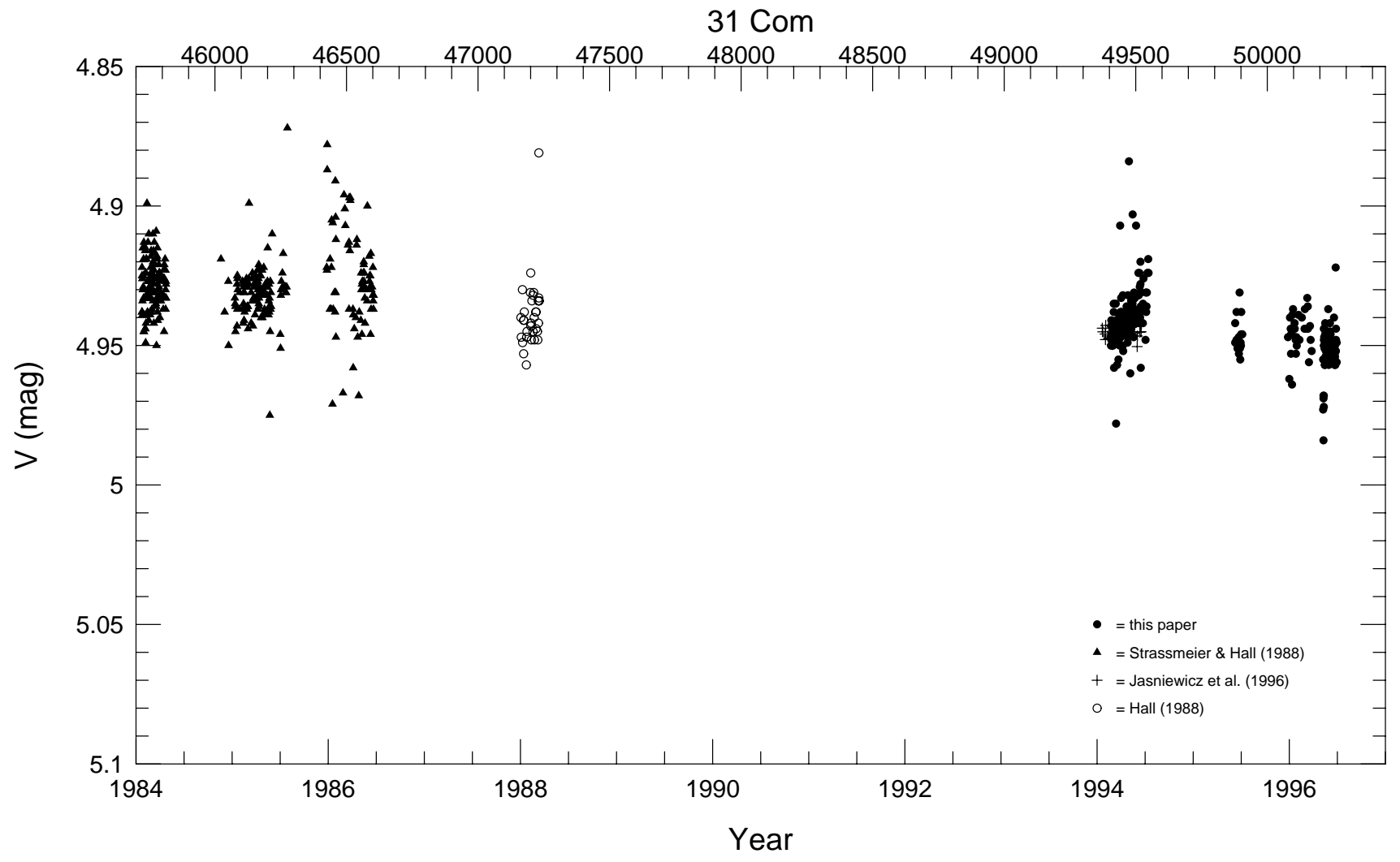

Fig. 15. HD $111812=31$ Com 


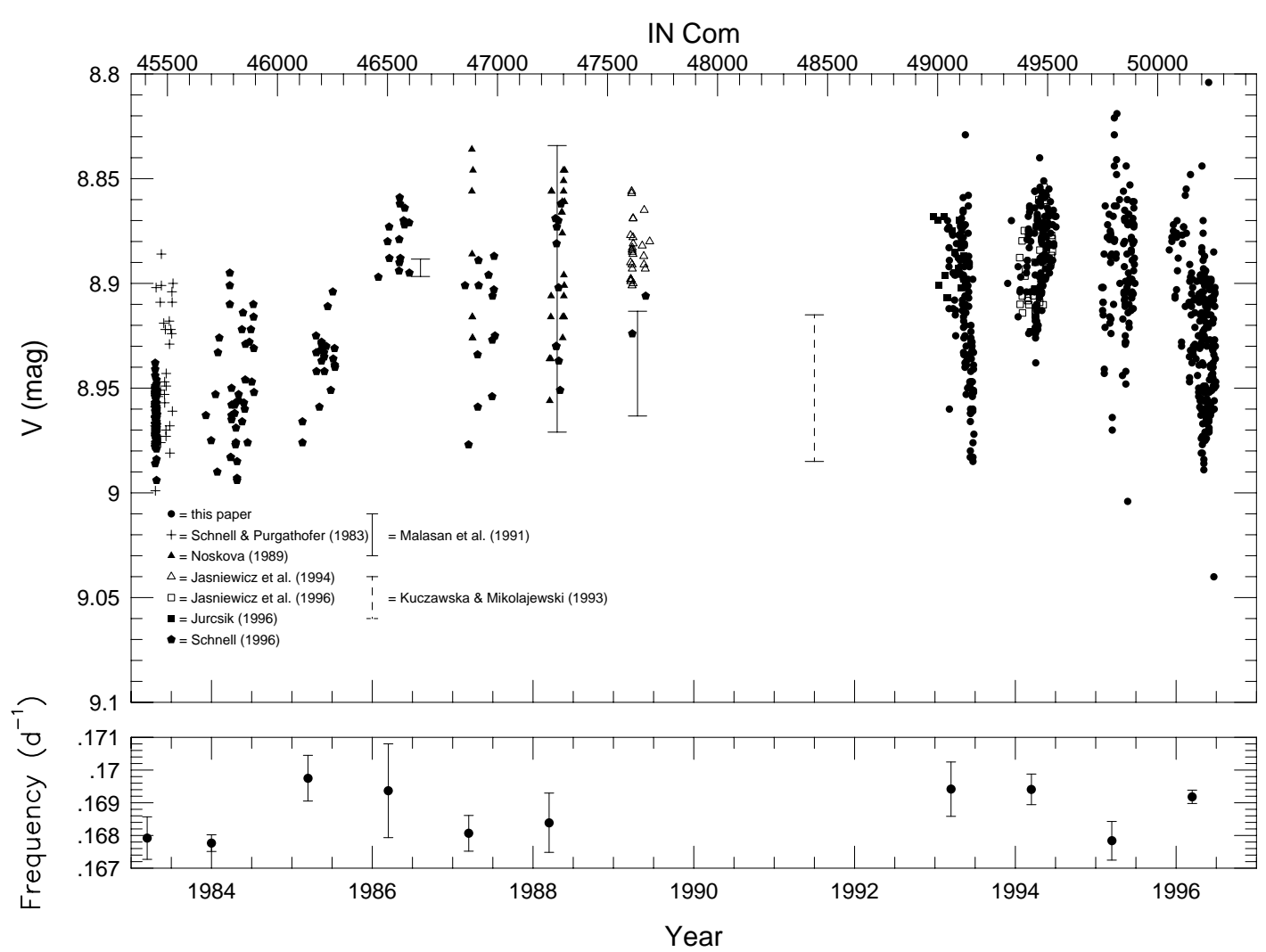

Fig. 16. HD $112313=$ IN Com

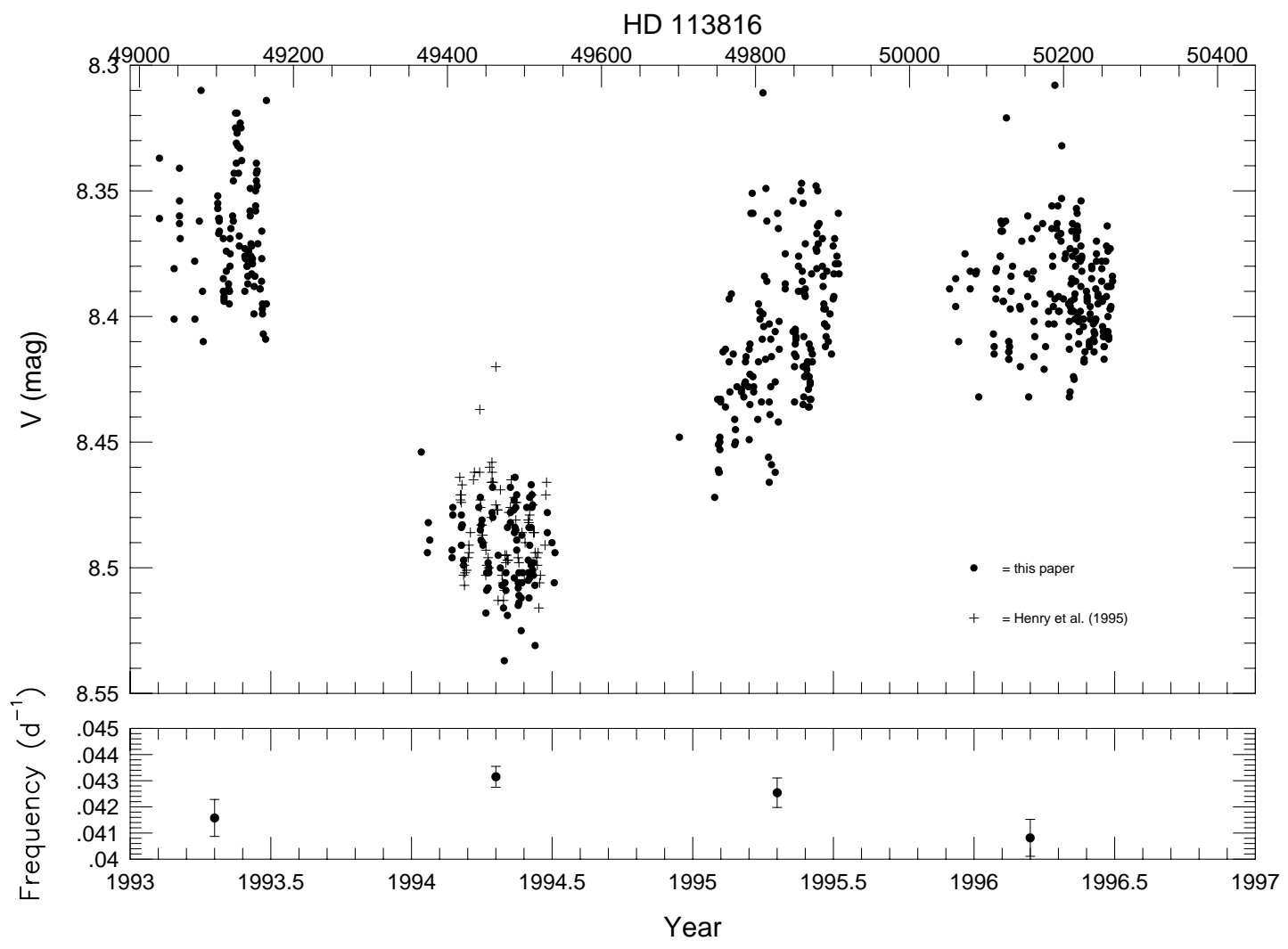

Fig. 17. HD 113816 


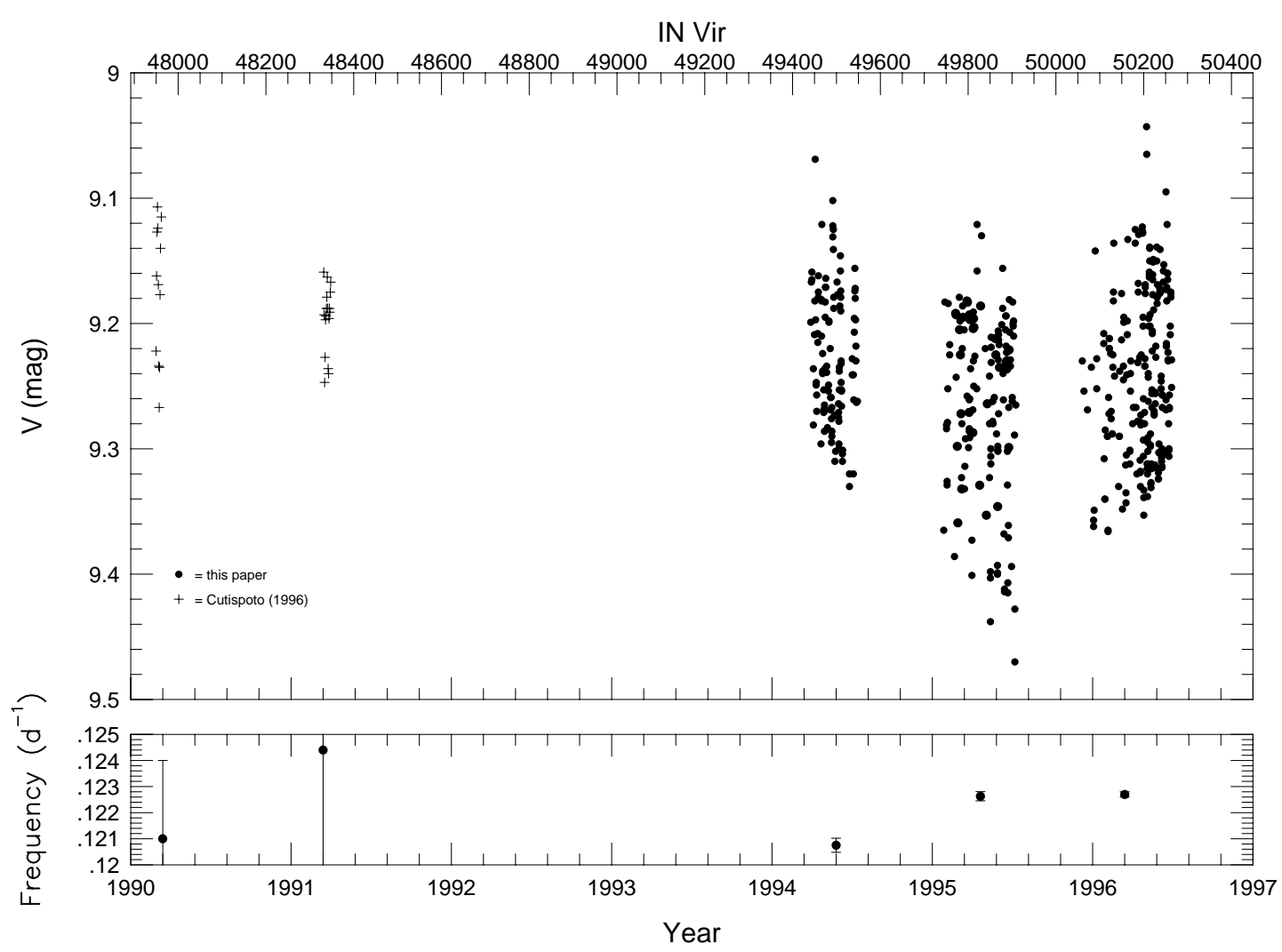

Fig. 18. HD $116544=$ IN Vir

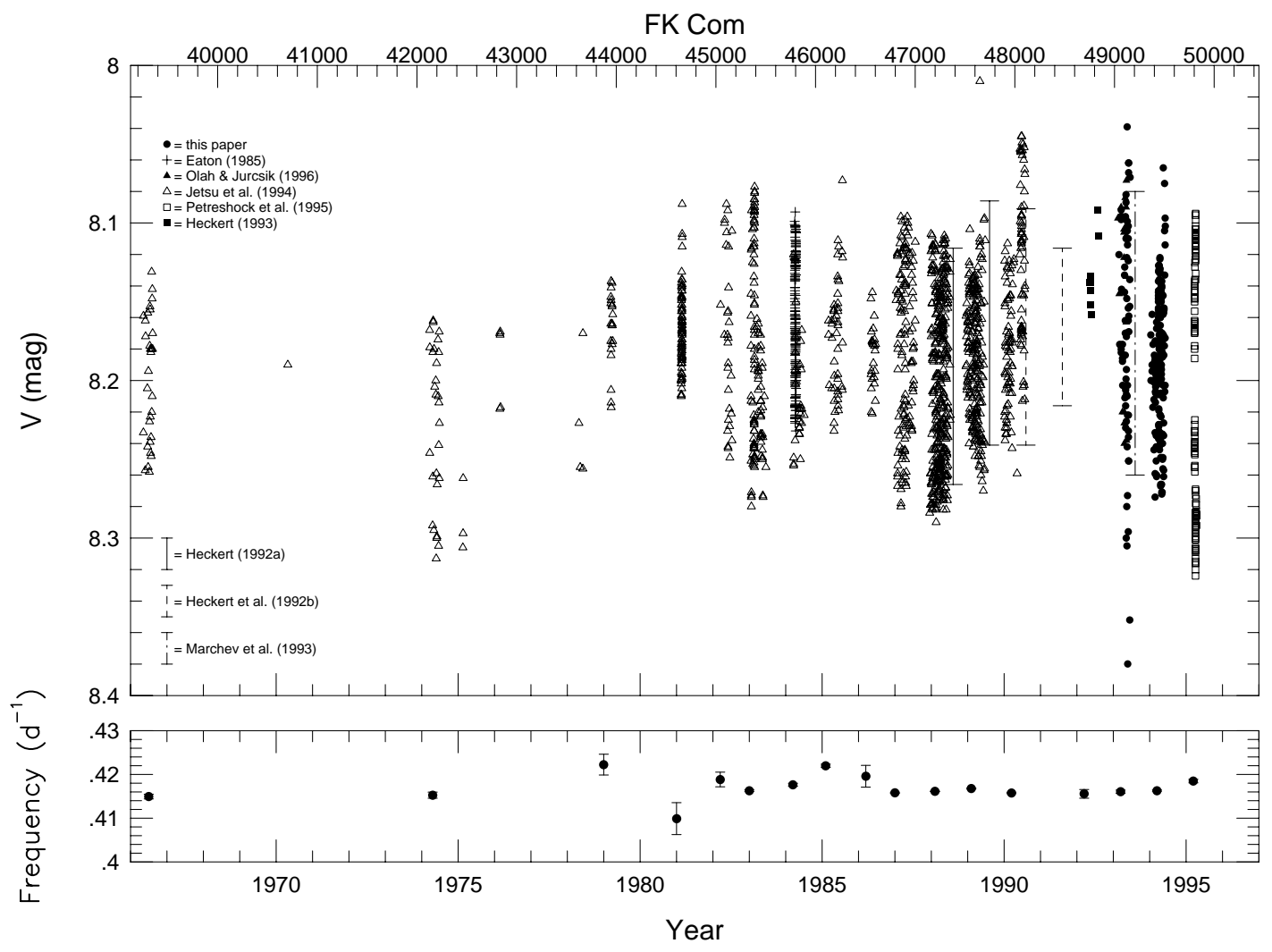

Fig. 19. HD $117555=$ FK Com 


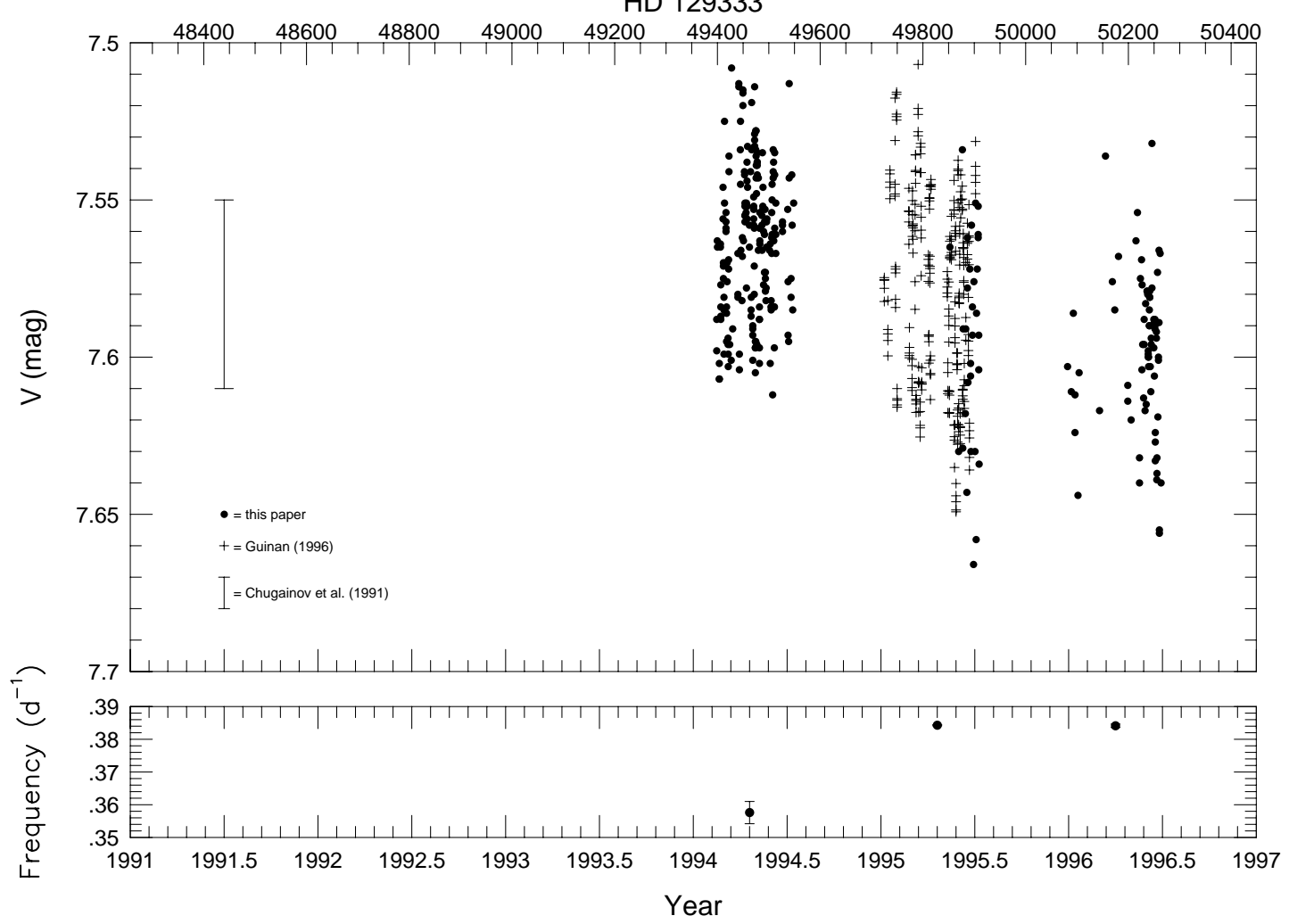

Fig. 20. HD 129333 = EK Dra

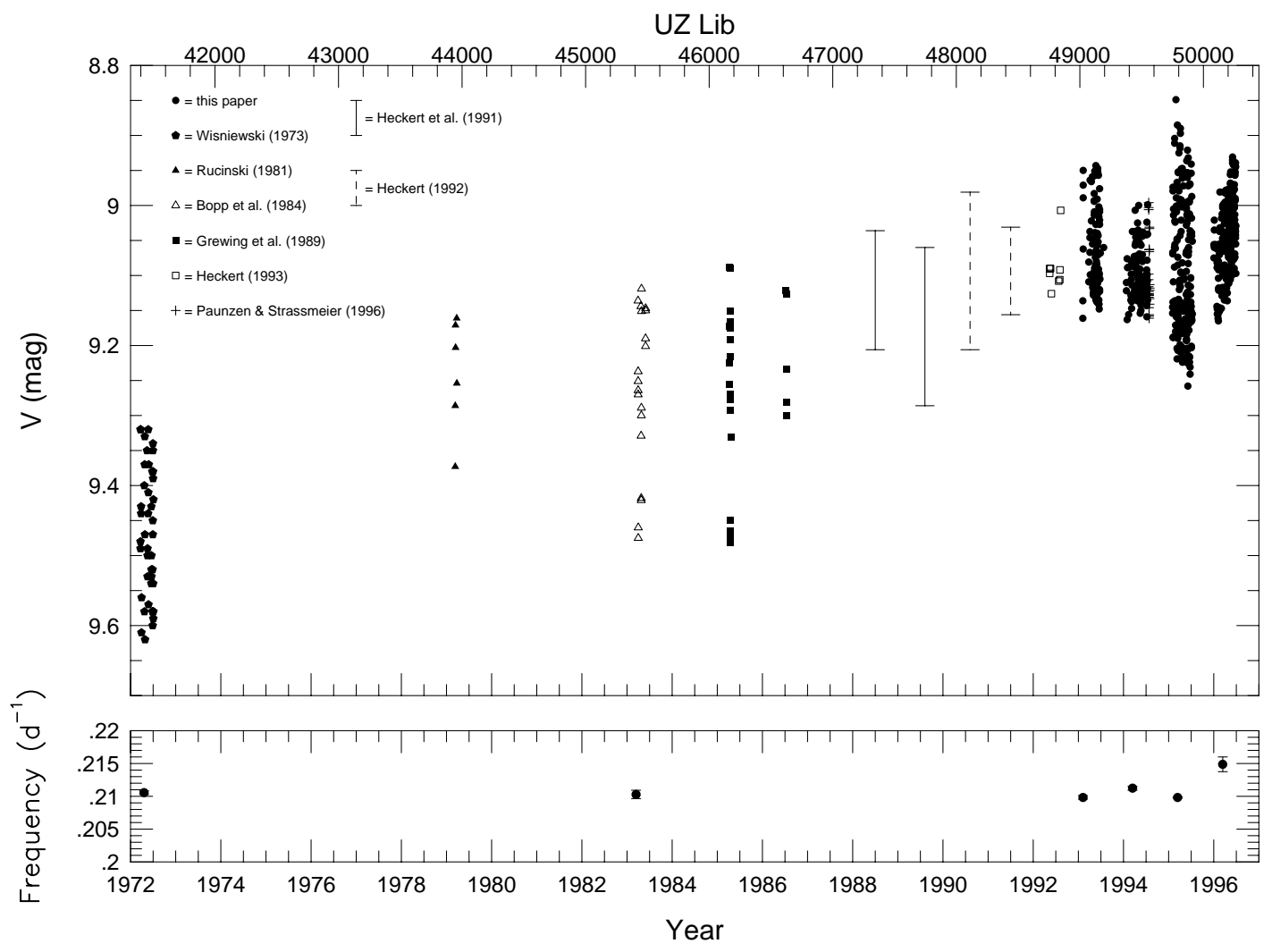

Fig. 21. UZ Lib 


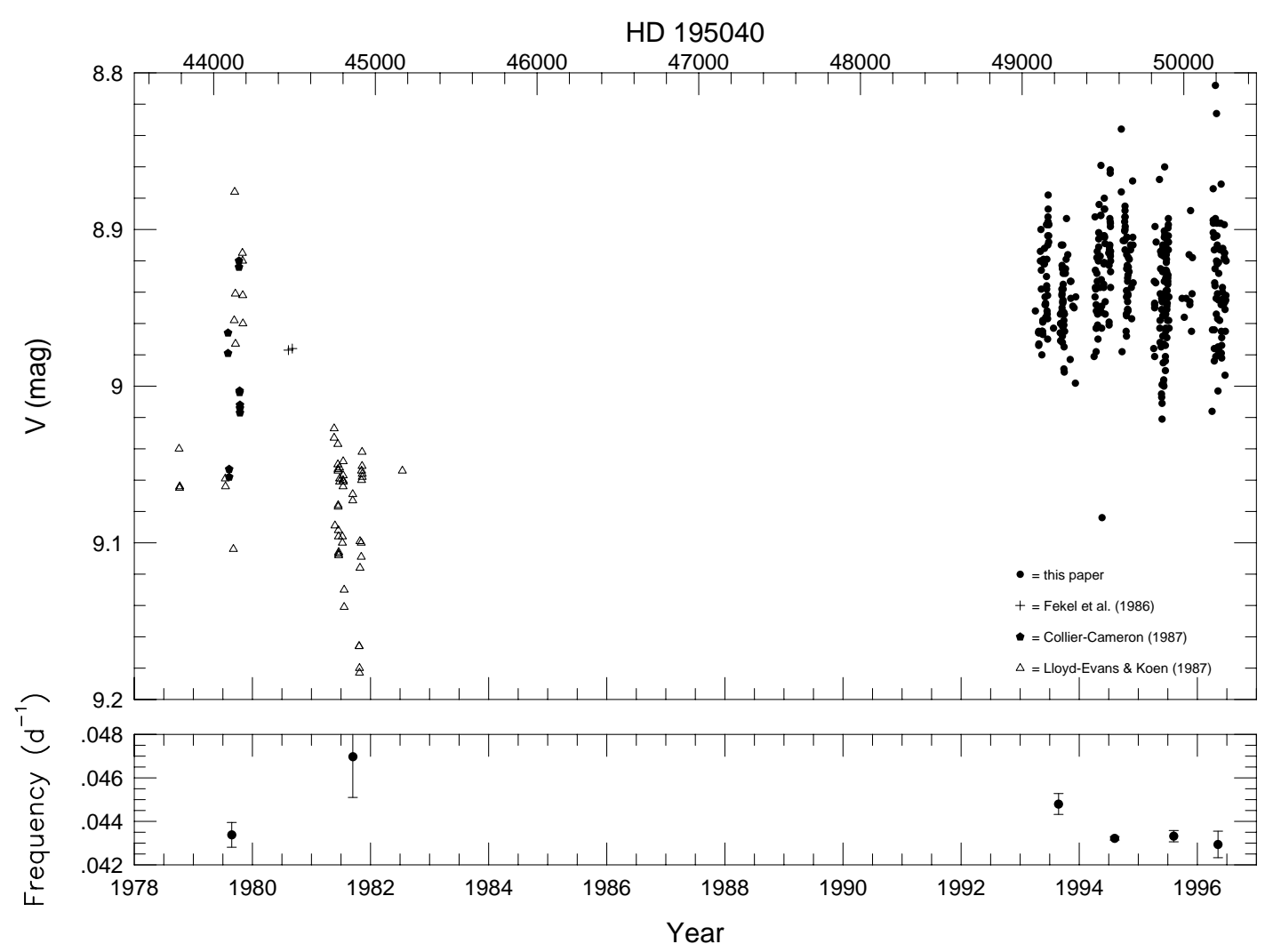

Fig. 22. HD $195040=$ AT Cap

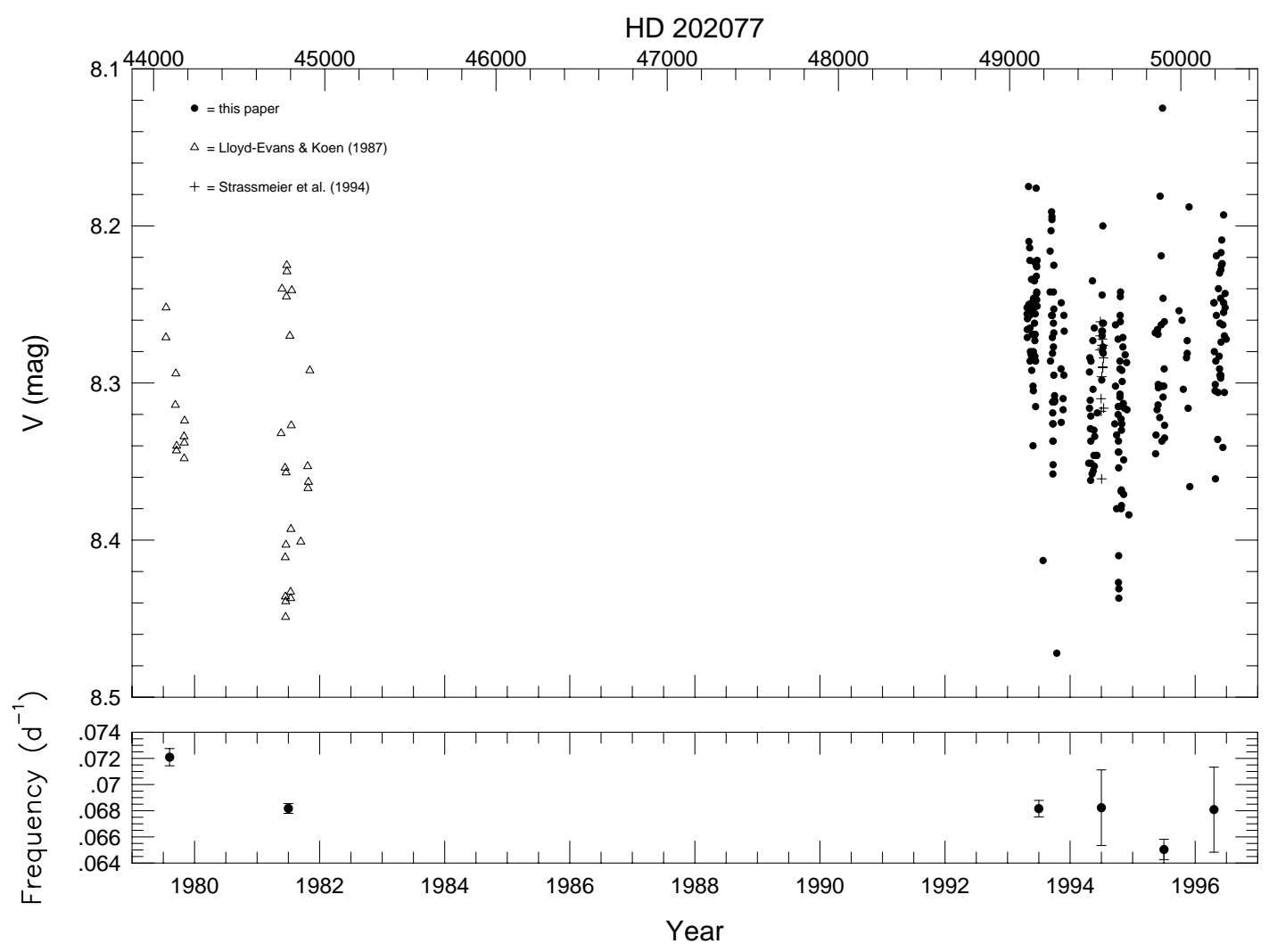

Fig. 23. HD $202077=\mathrm{BM}$ Mic 

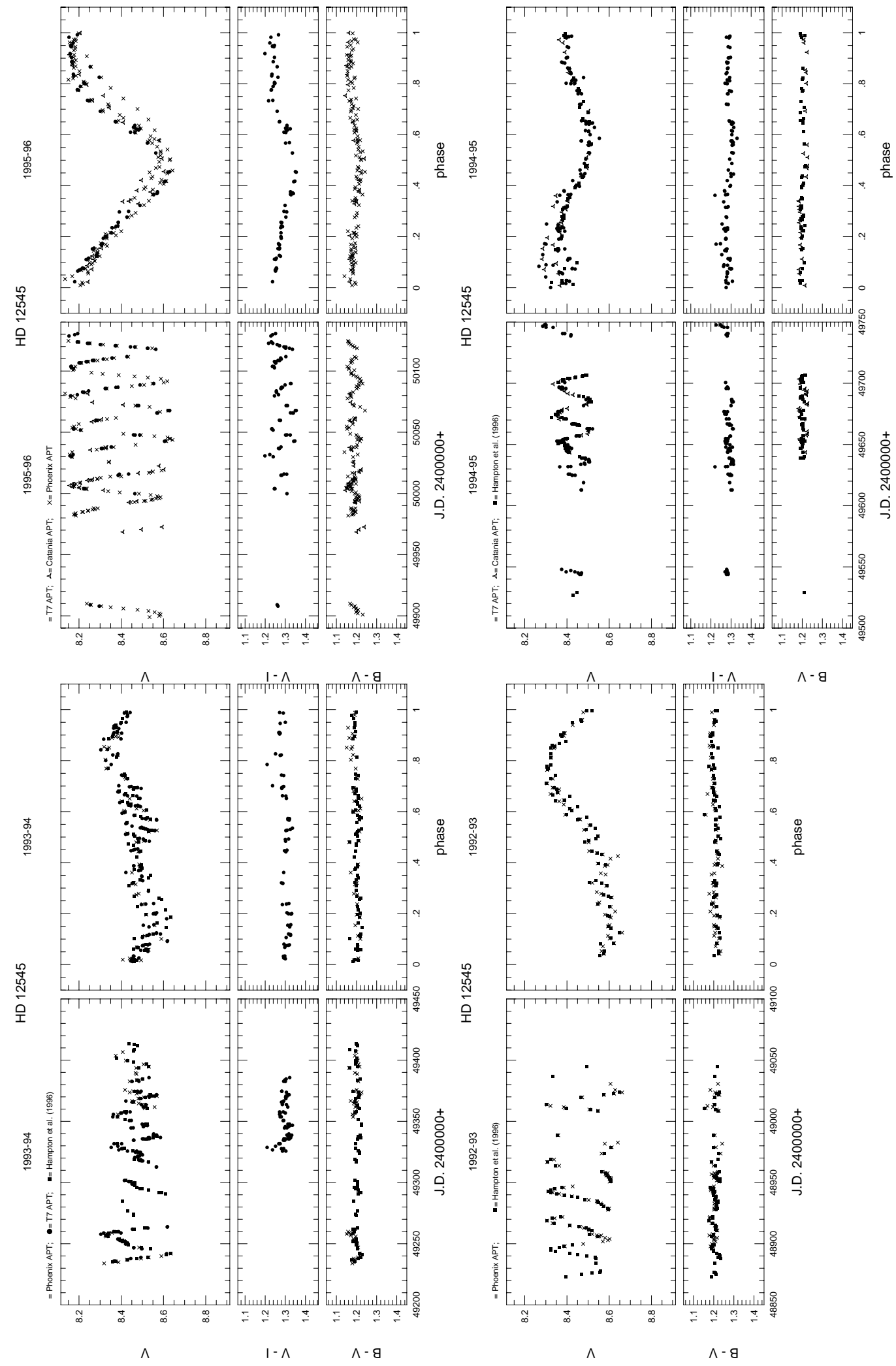

Fig. 26. HD $12545=\mathrm{XX}$ Tri 


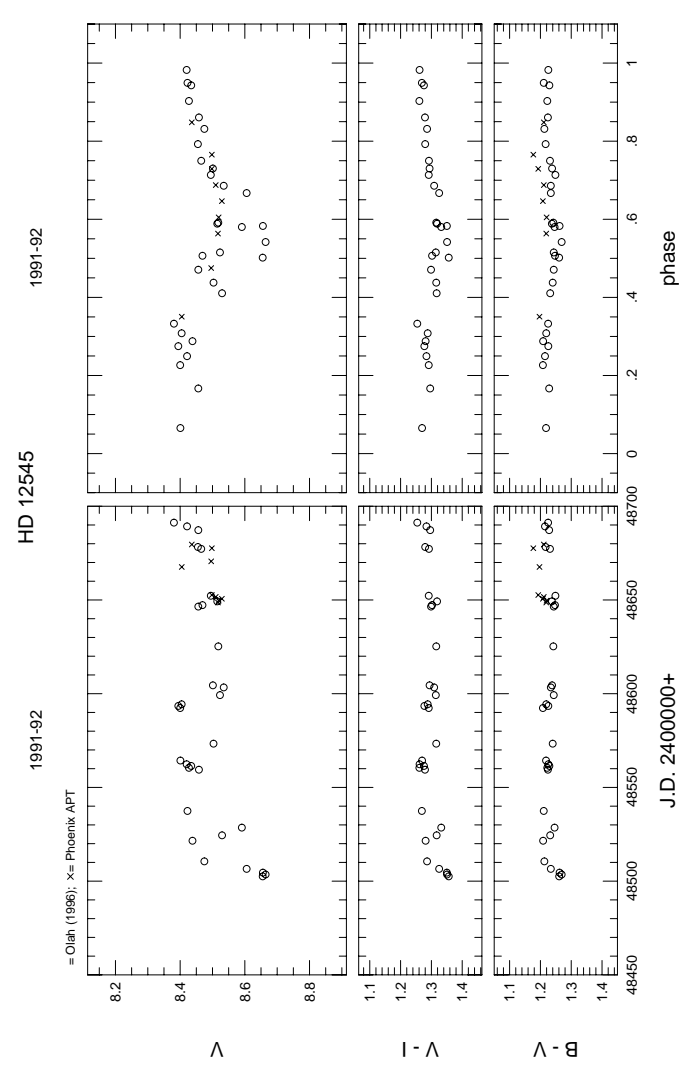

Fig. 26. continued
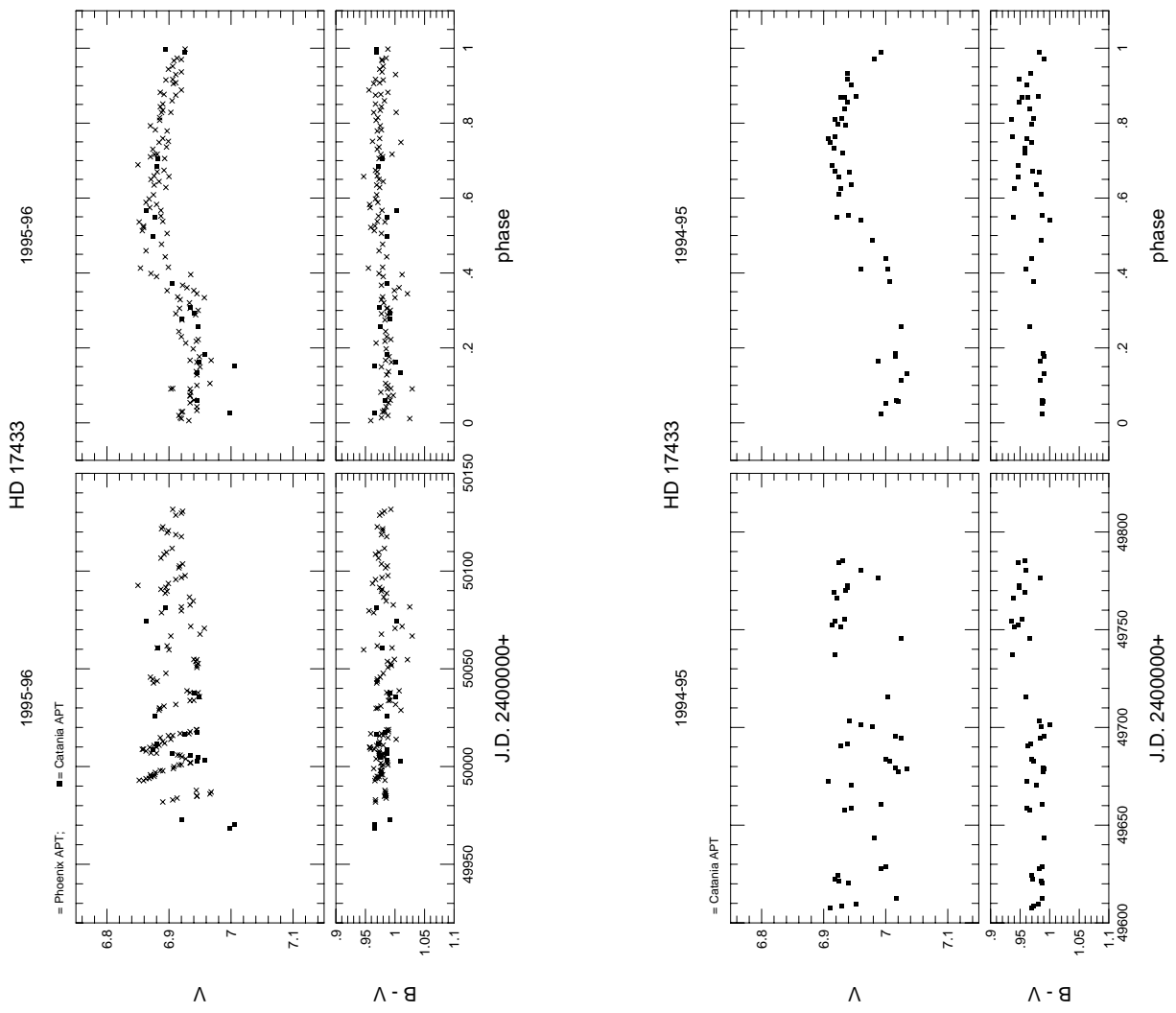

Fig. 27. HD $17433=$ VY Ari 

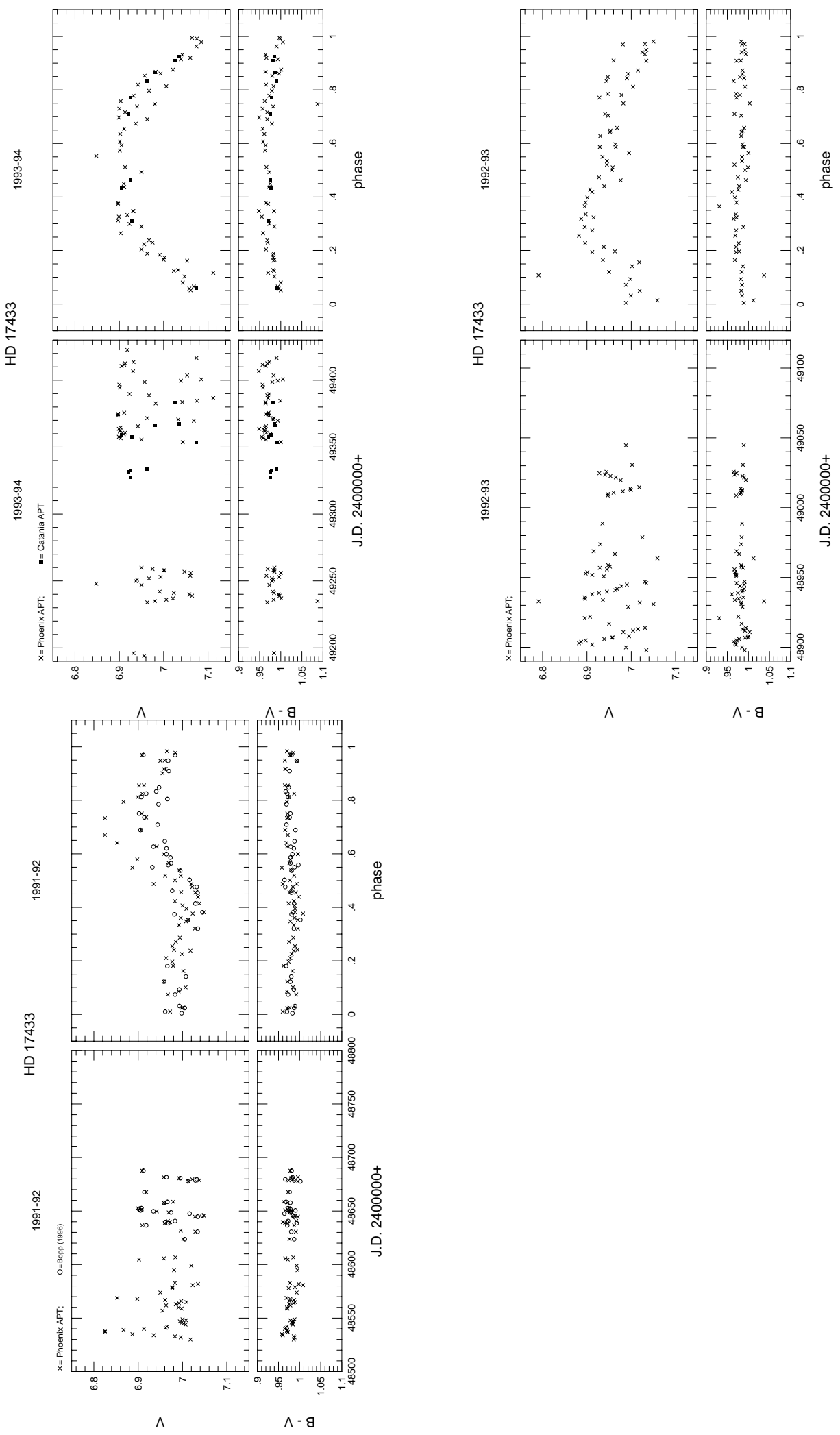

Fig. 27. continued 

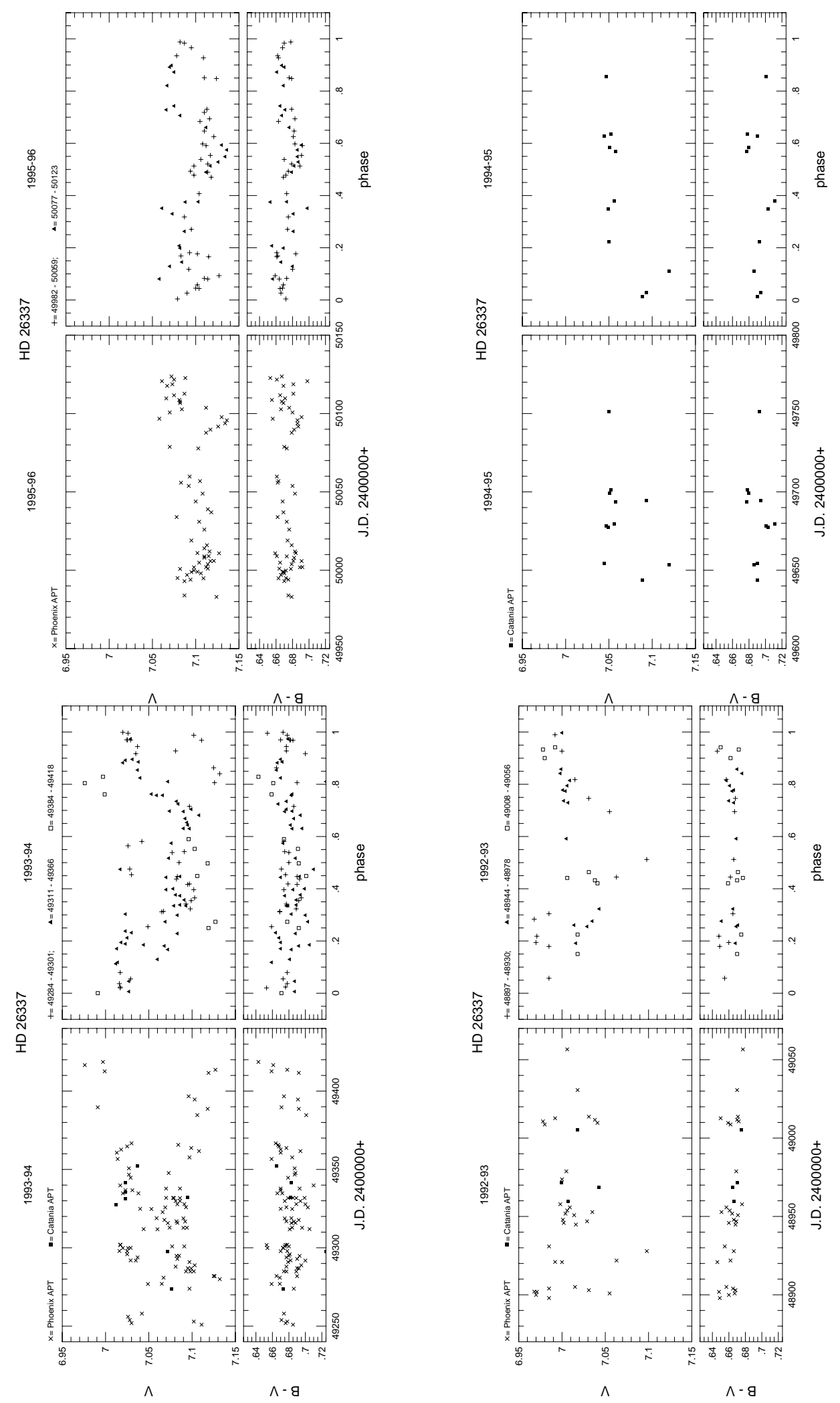

Fig. 28. HD $26337=$ EI Eri 


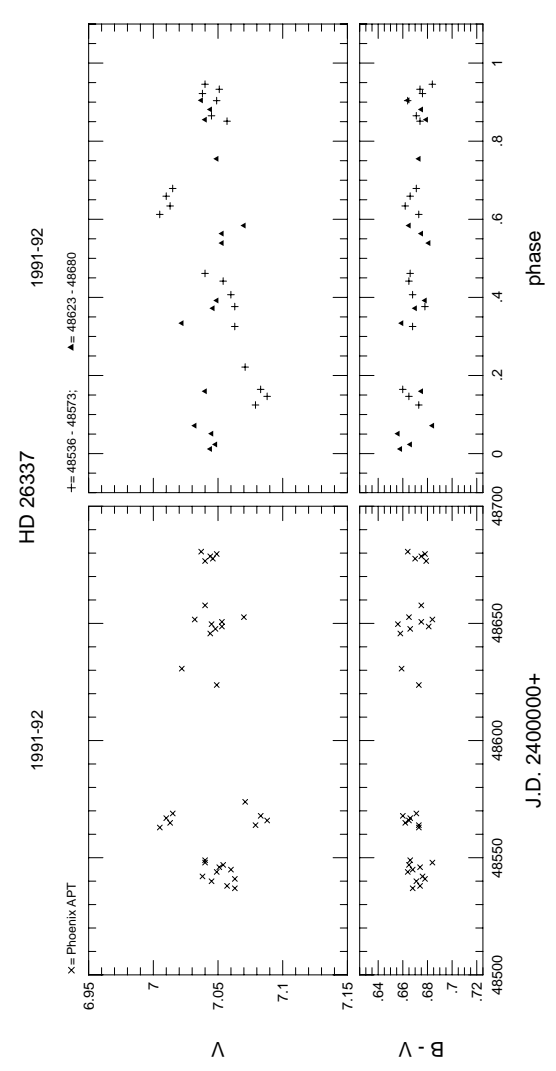

Fig. 28. continued
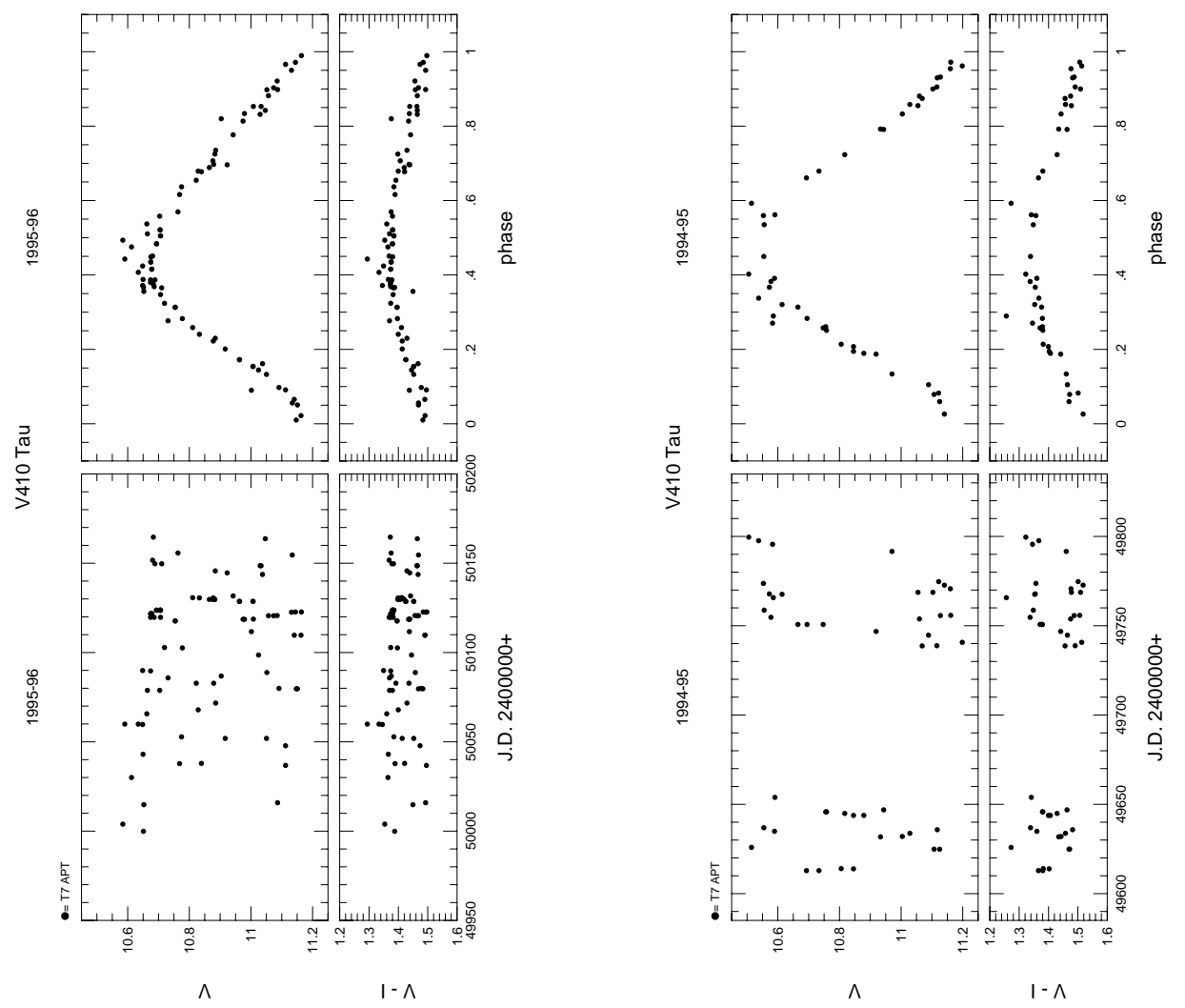

Fig. 29. V410 Tau 

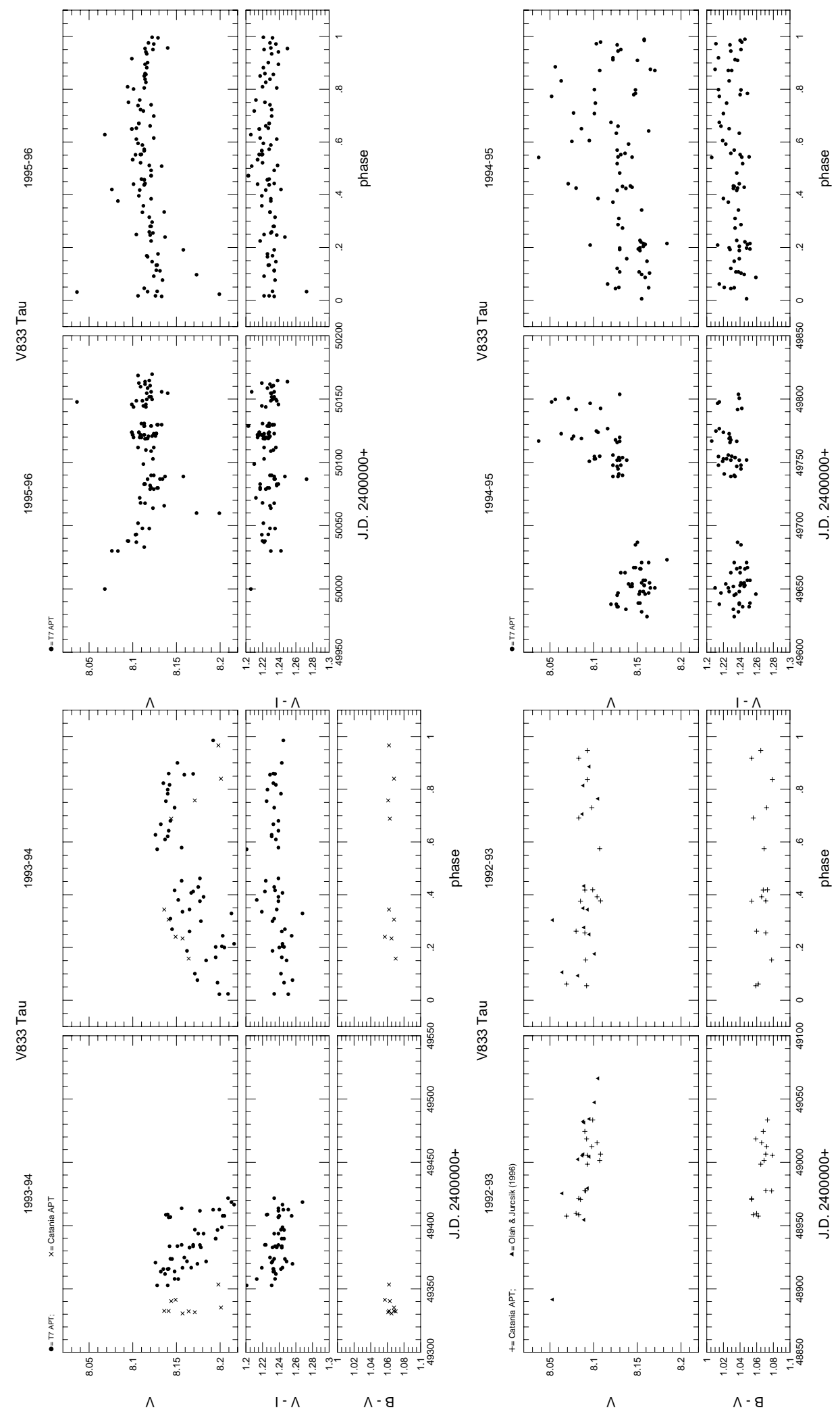

Fig. 30. HD $283750=$ V 833 Tau 


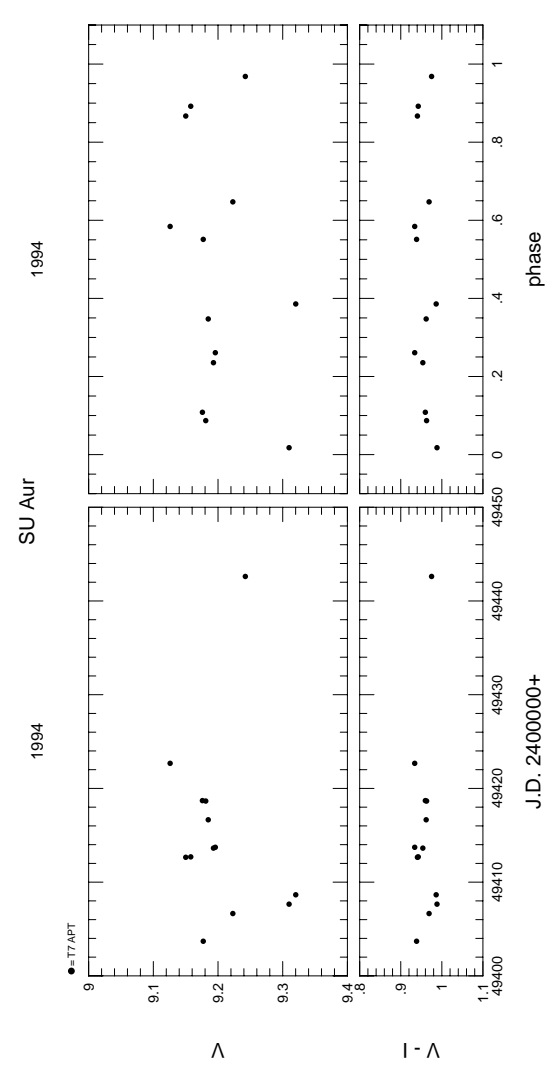

Fig. 31. SU Aur
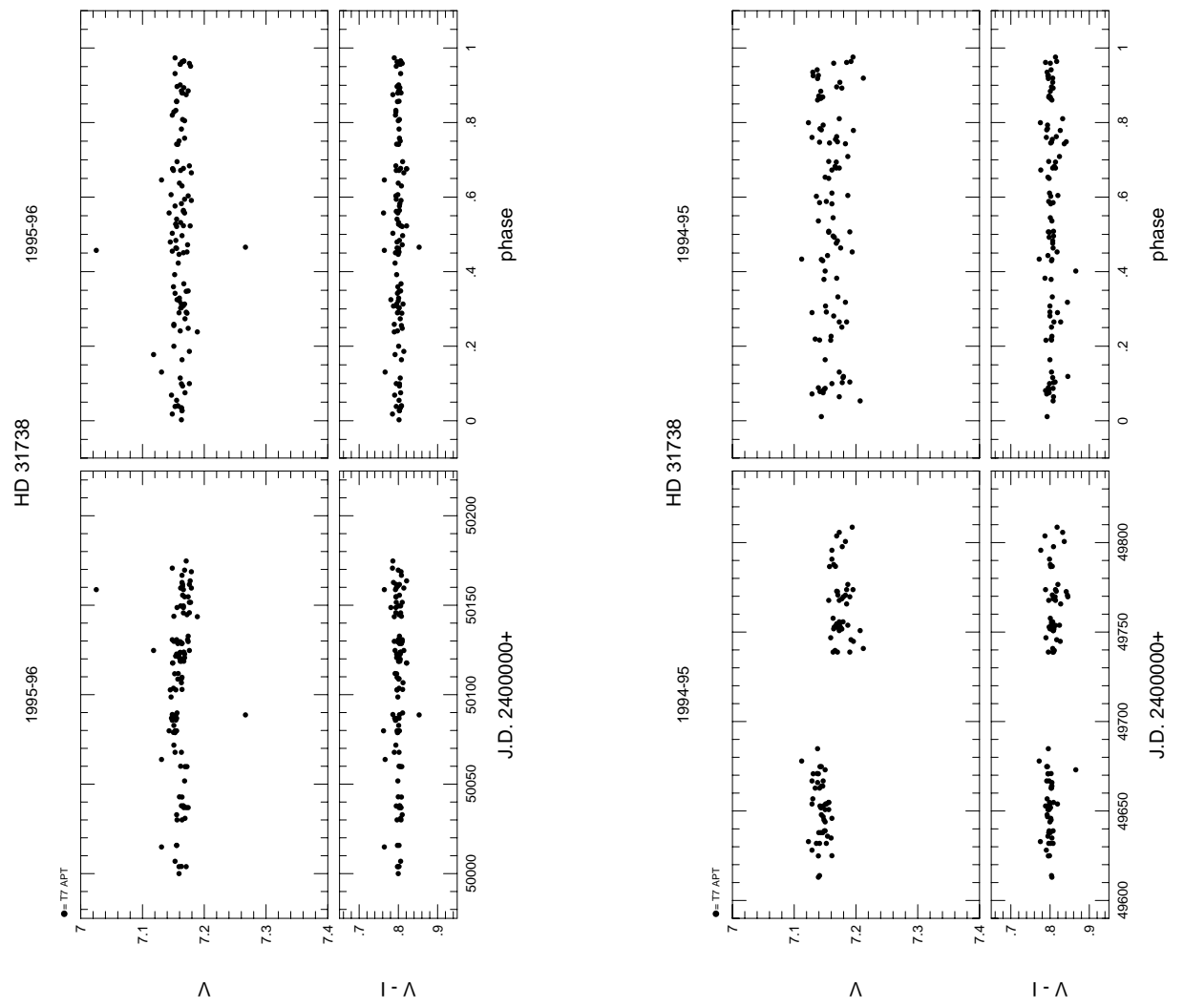

Fig. 32. HD $31738=$ V1198 Ori 


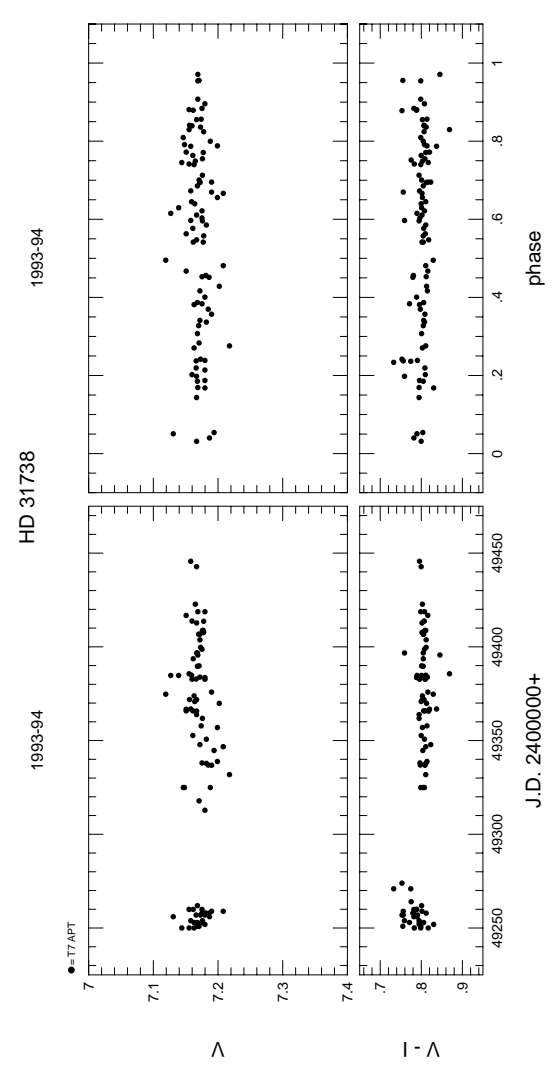

Fig. 32. continued
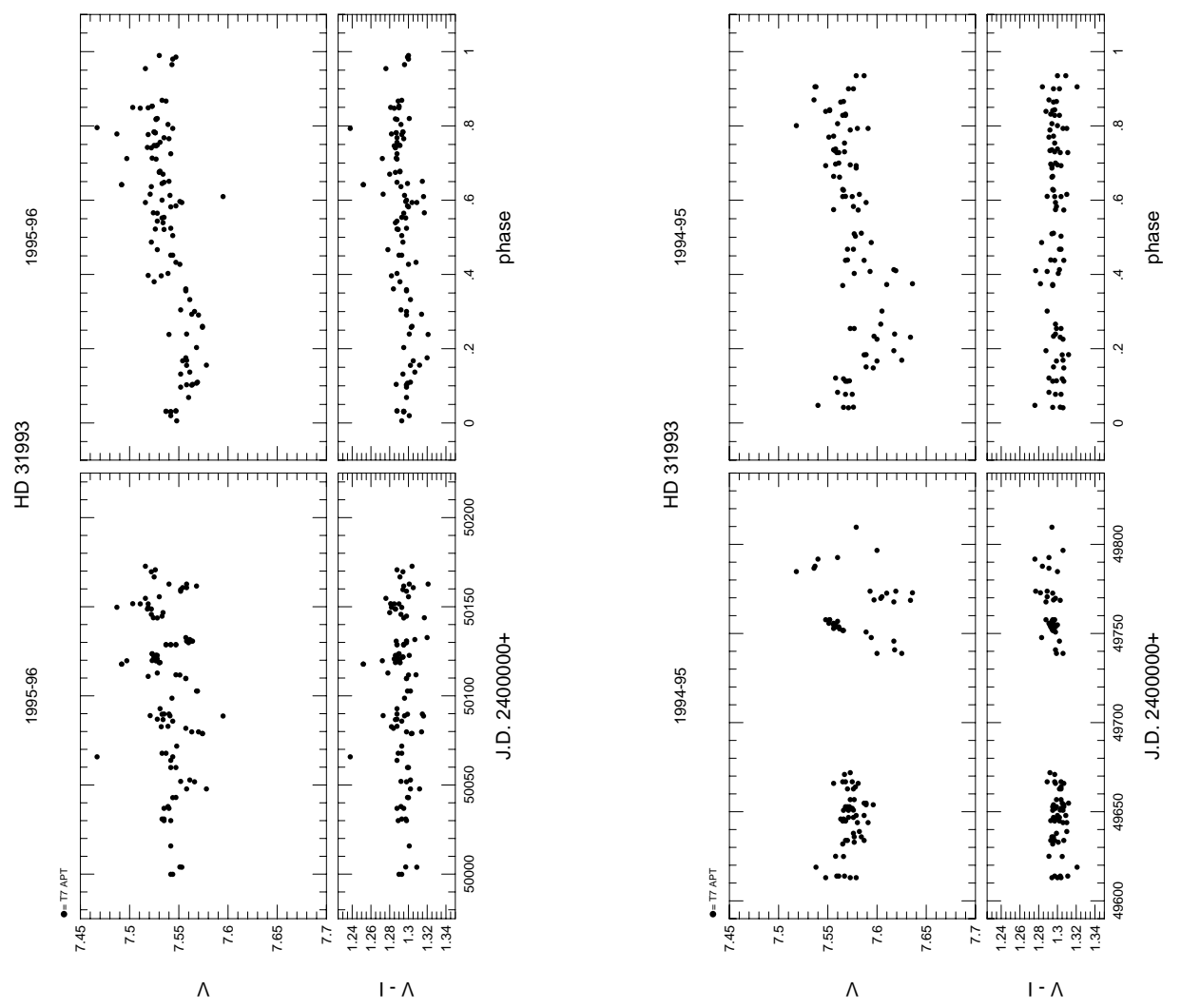

Fig. 33. HD $31993=$ V1192 Ori 


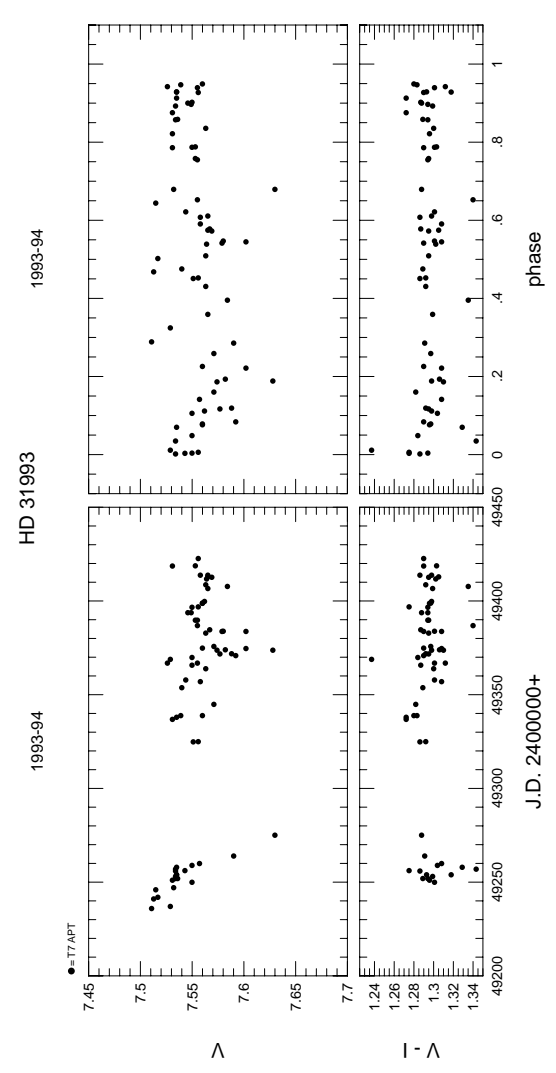

Fig. 33. continued
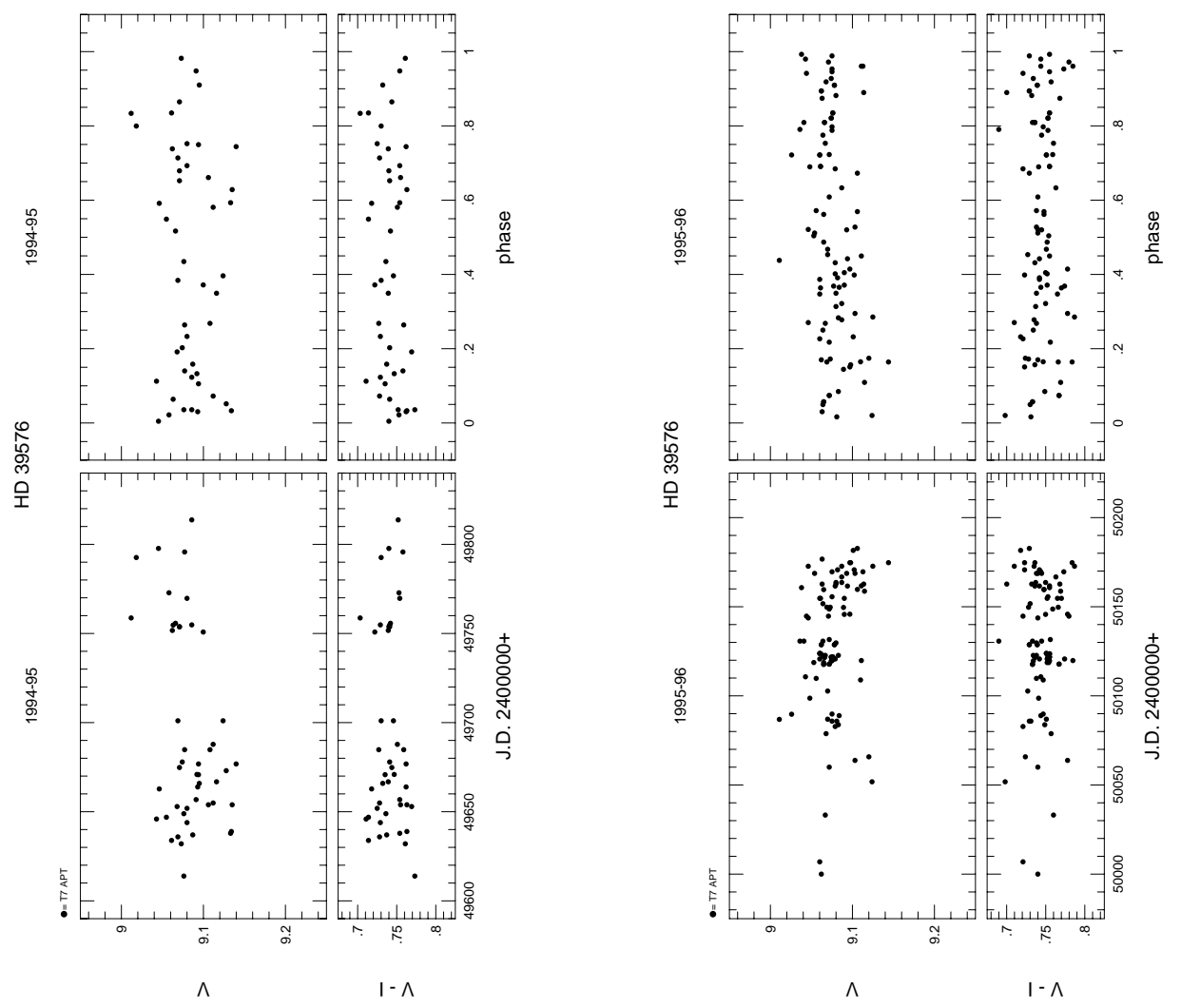

Fig. 34. HD 39576 


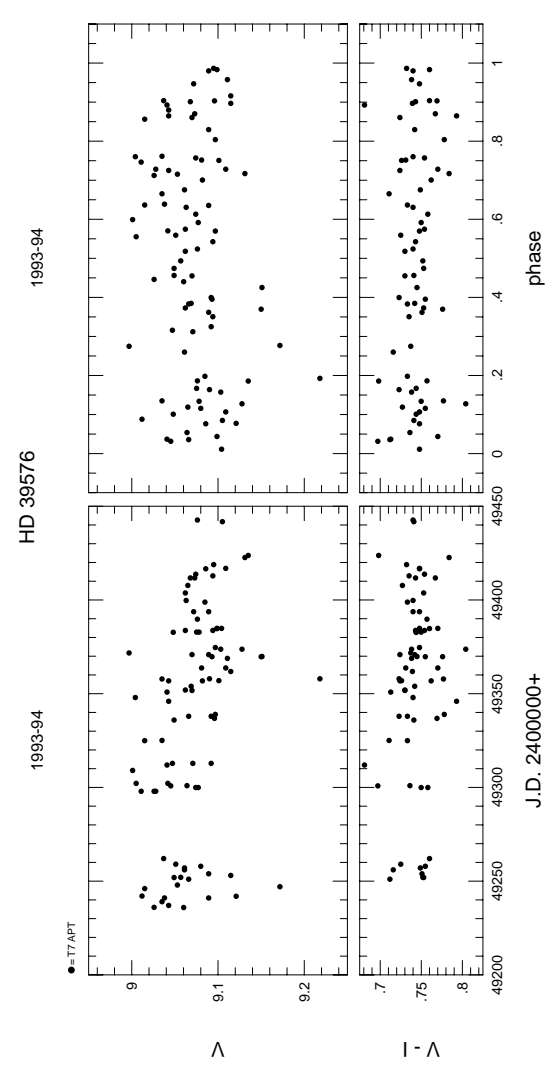

Fig. 34. continued
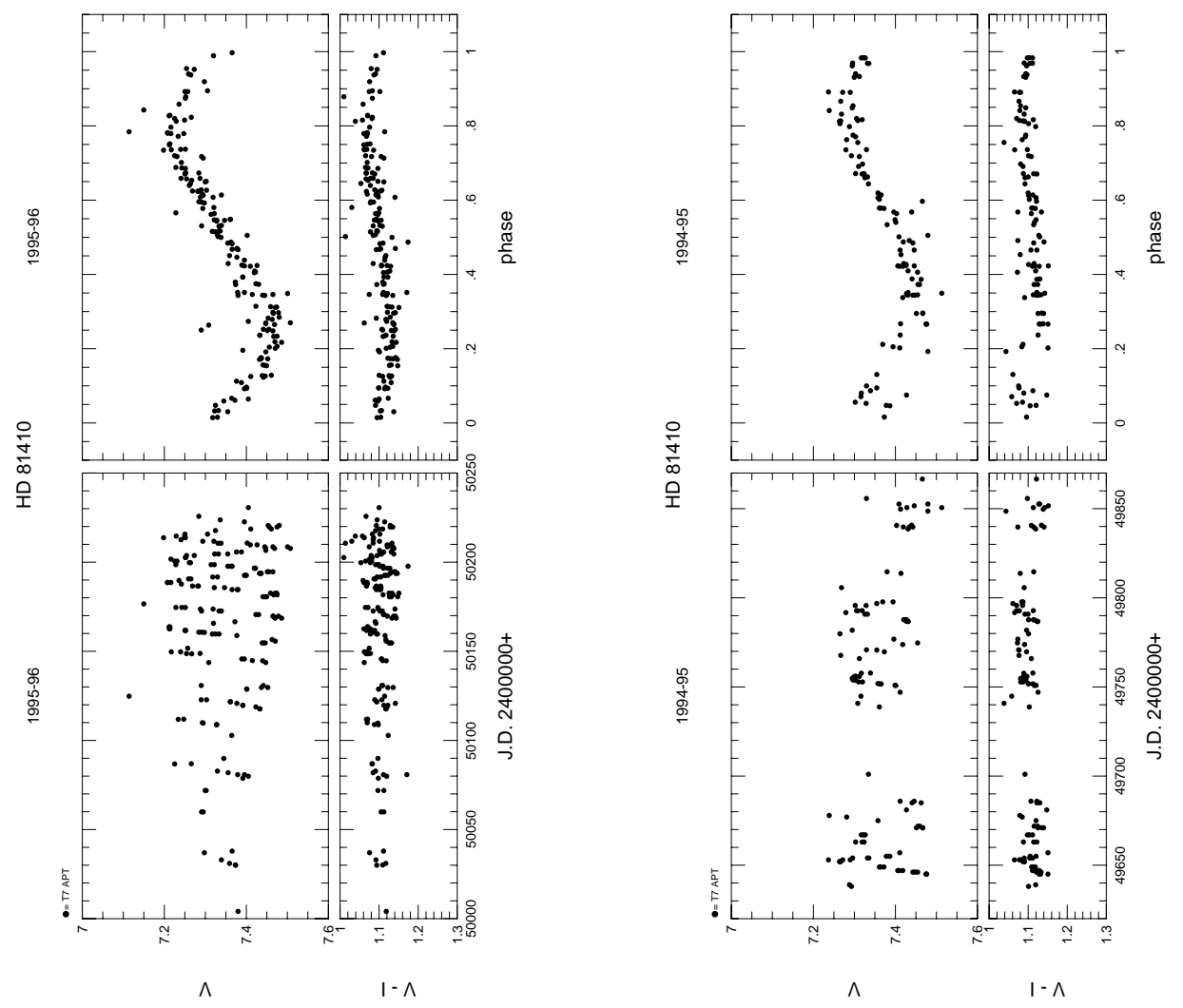

Fig. 35. HD $81410=$ IL Hya 

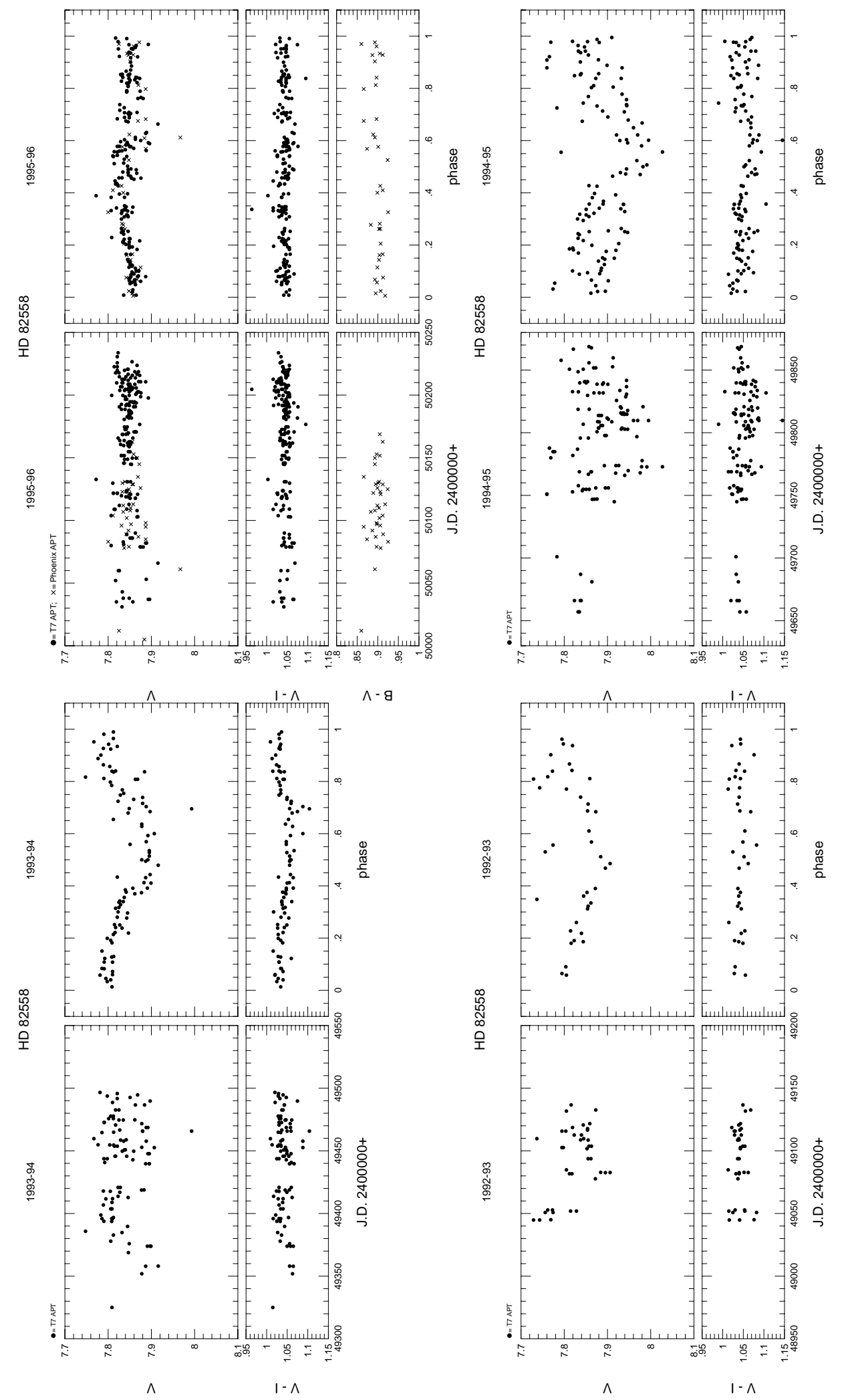

Fig. 37. HD $82558=$ LQ Hya 

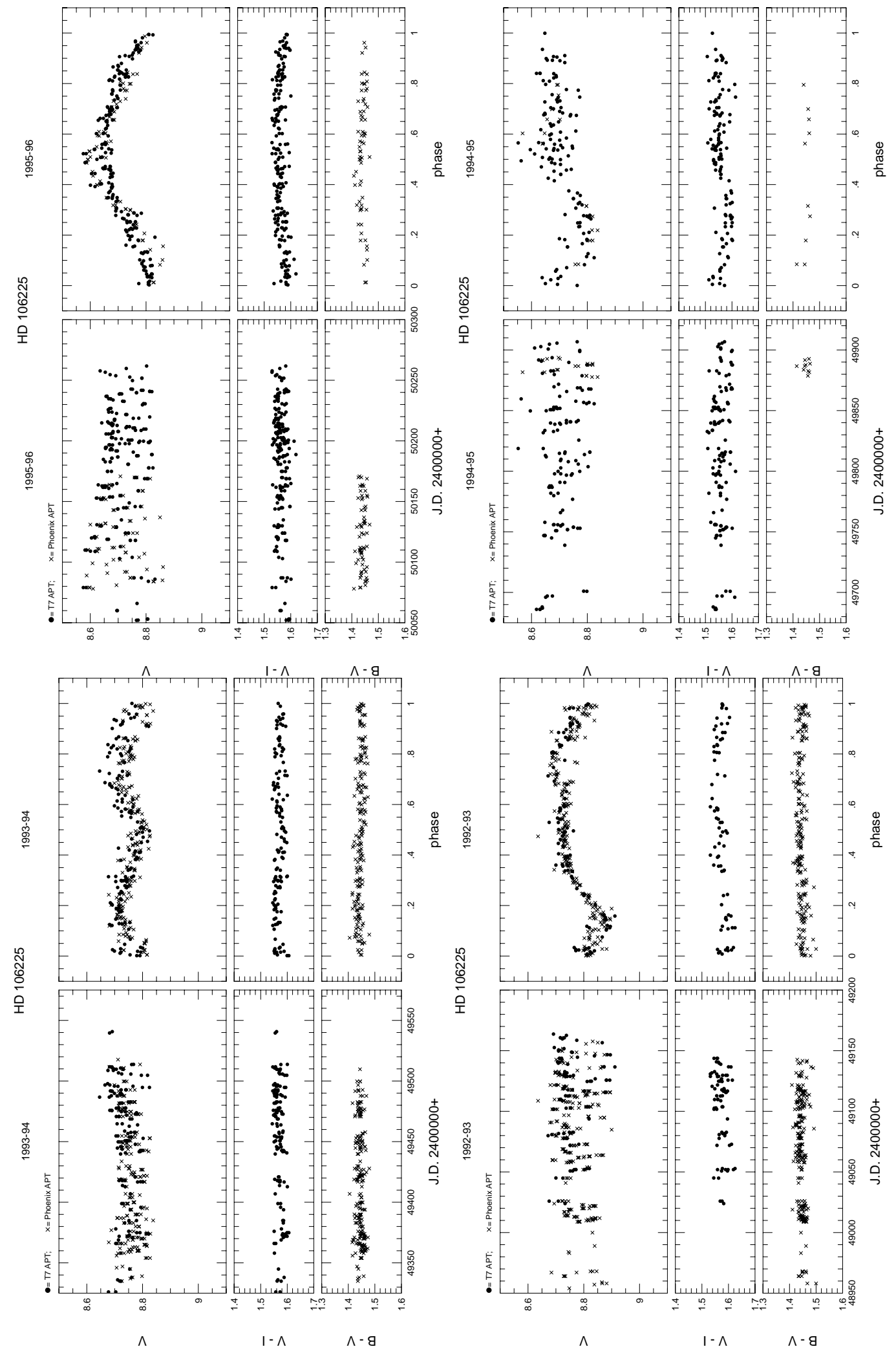

Fig. 38. HD $106225=$ HU Vir 

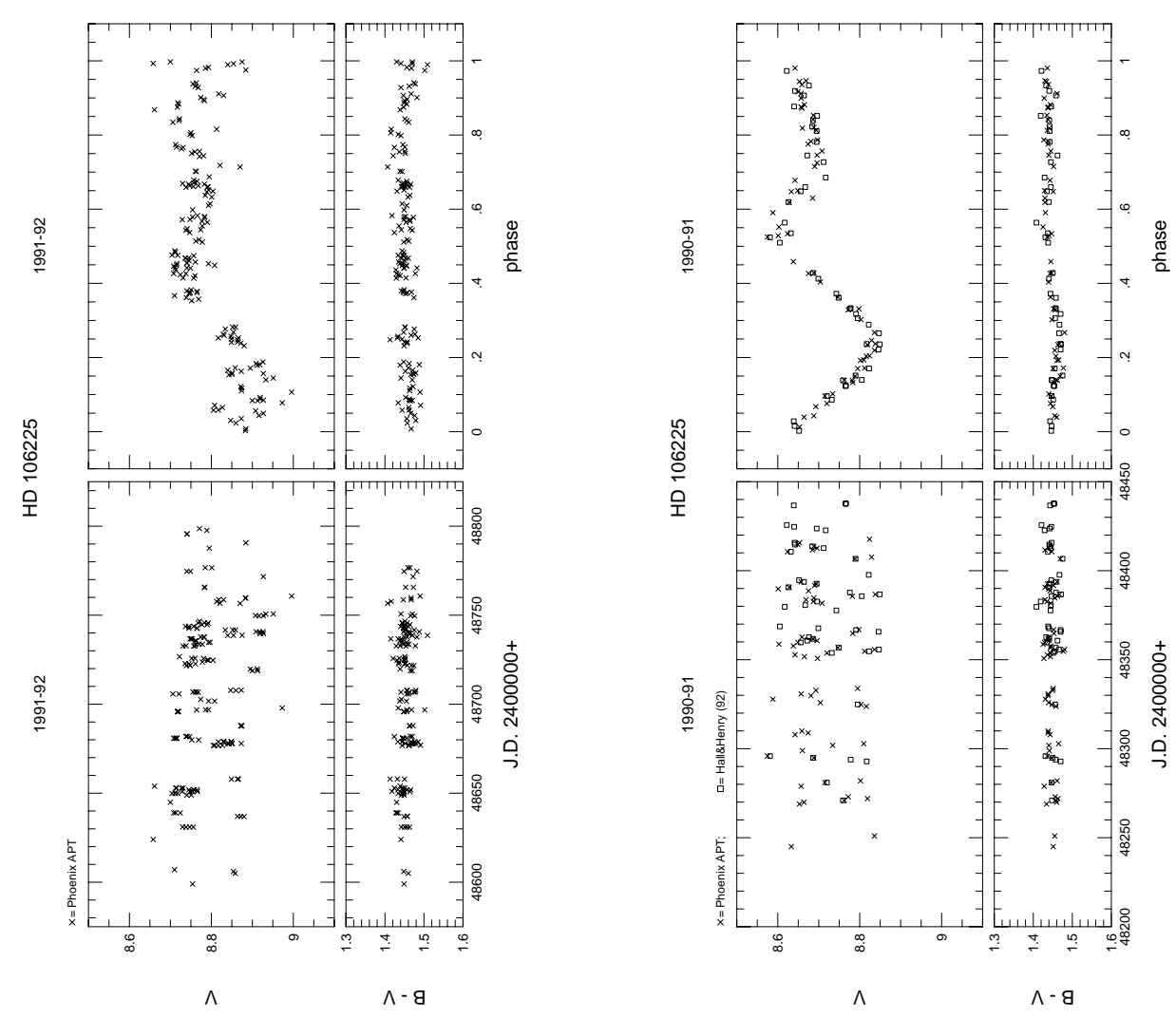

Fig. 38. continued
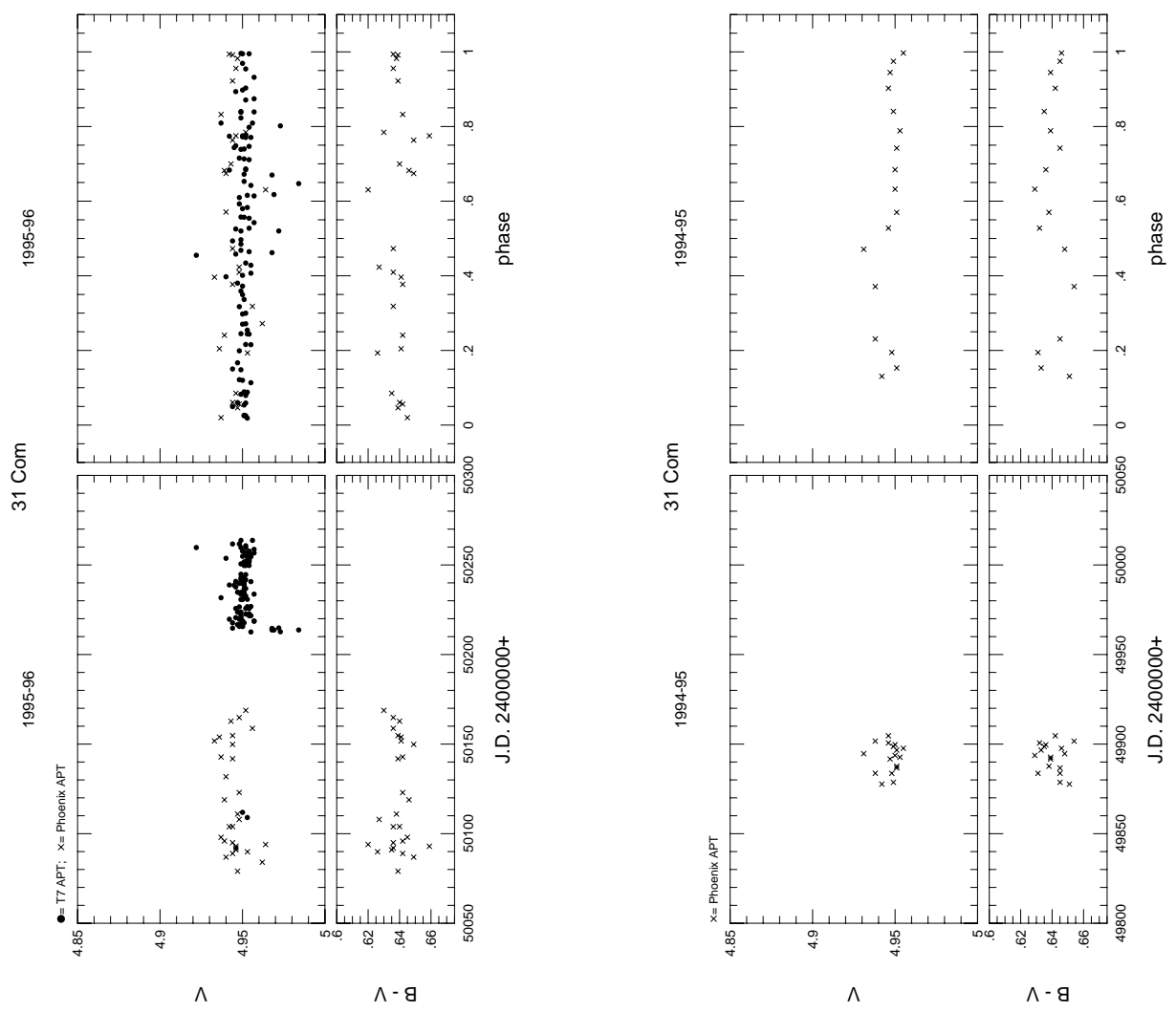

Fig. 39. HD $111812=31$ Com 


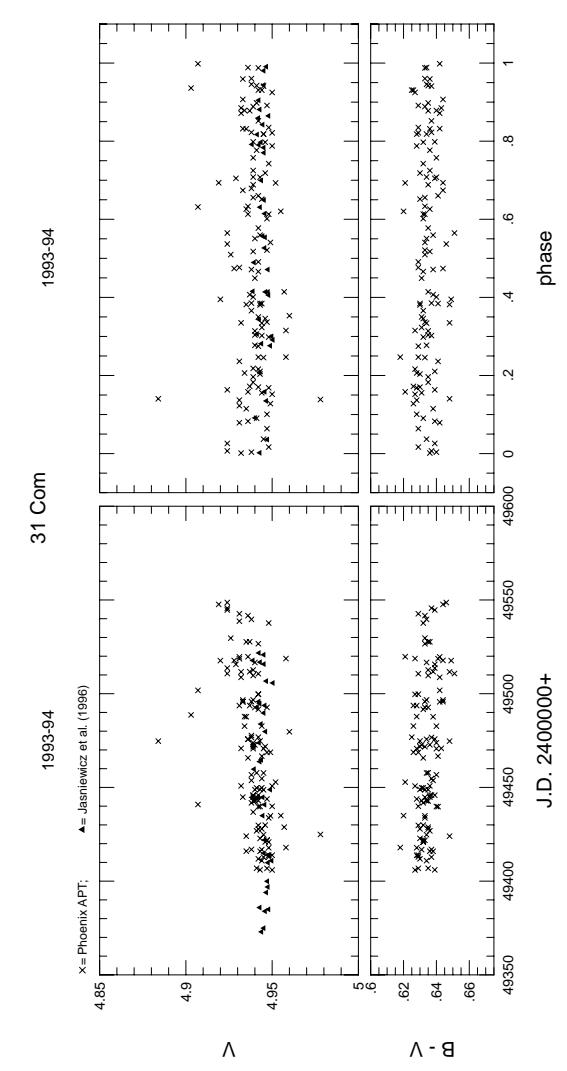

Fig. 39. continued
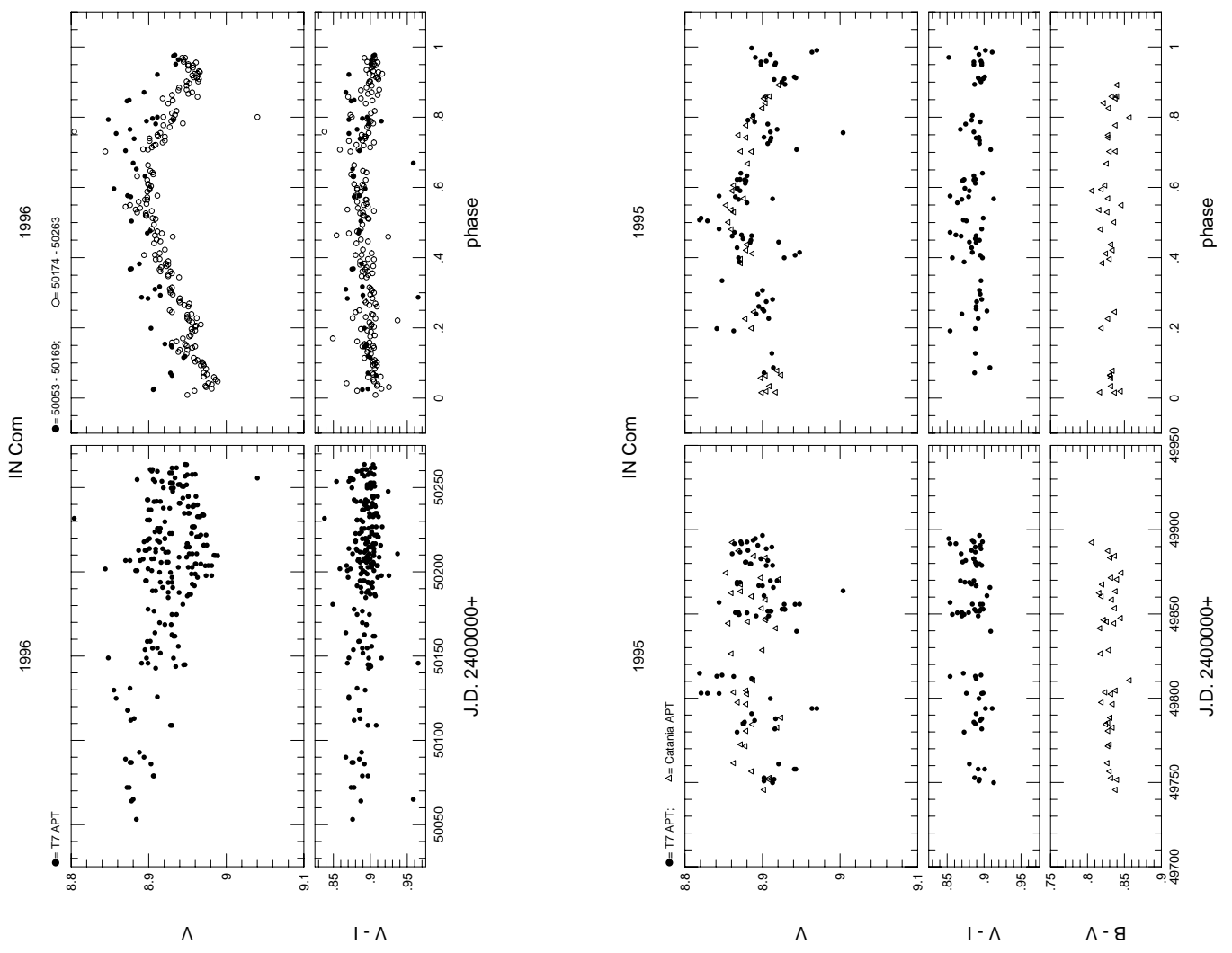

Fig. 40. HD $112313=$ IN Com 


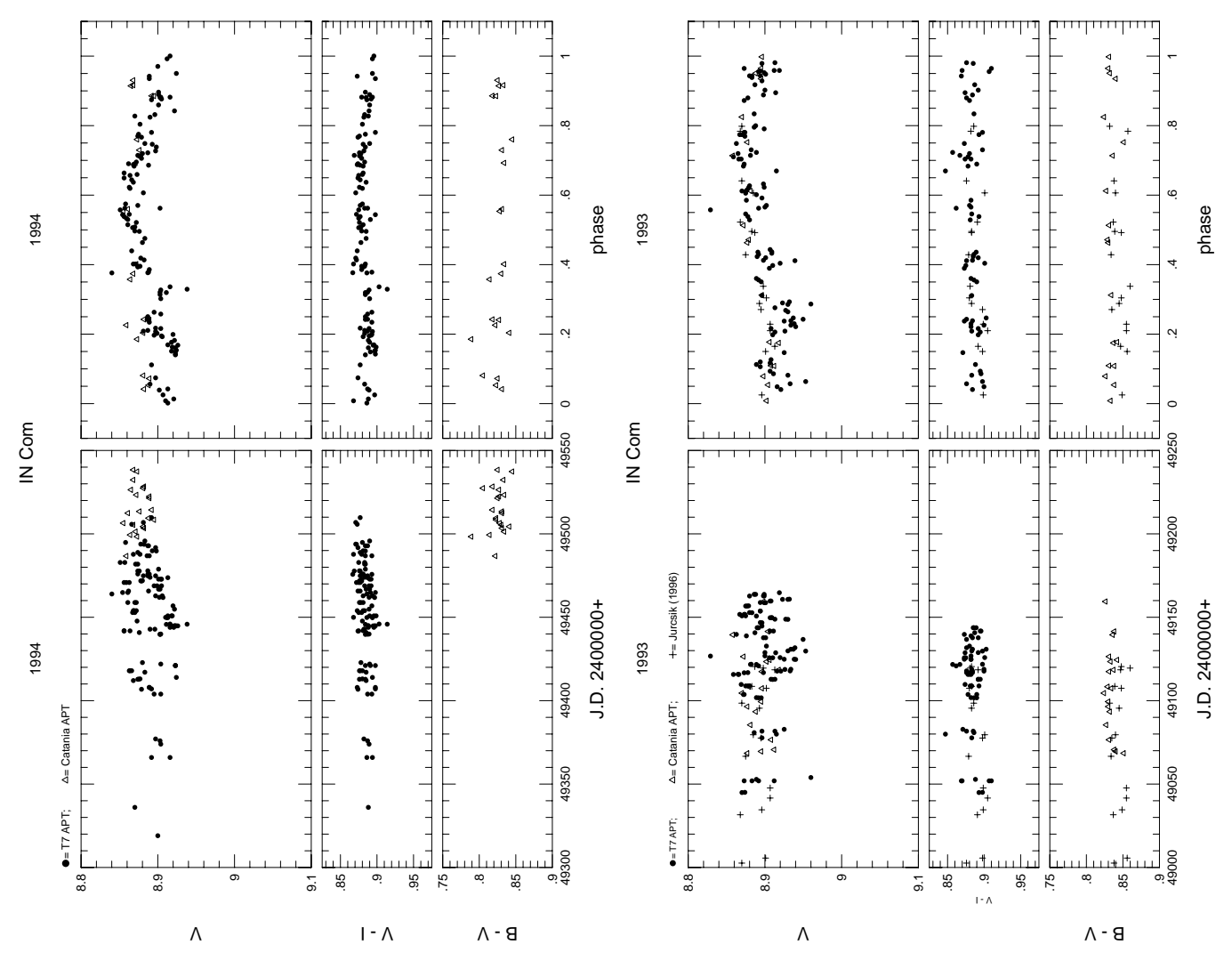

Fig. 40. continued
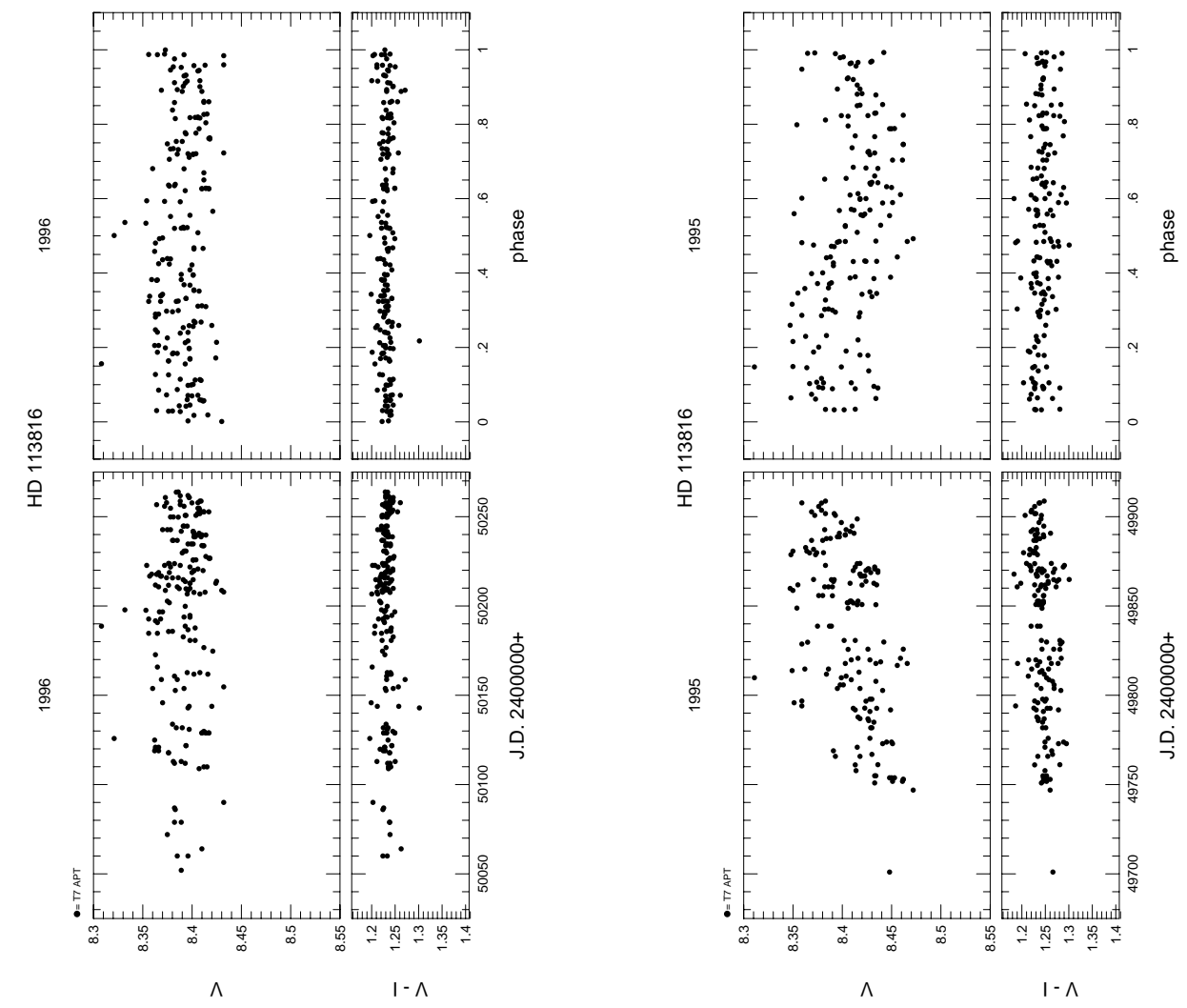

Fig. 41. HD 113816 

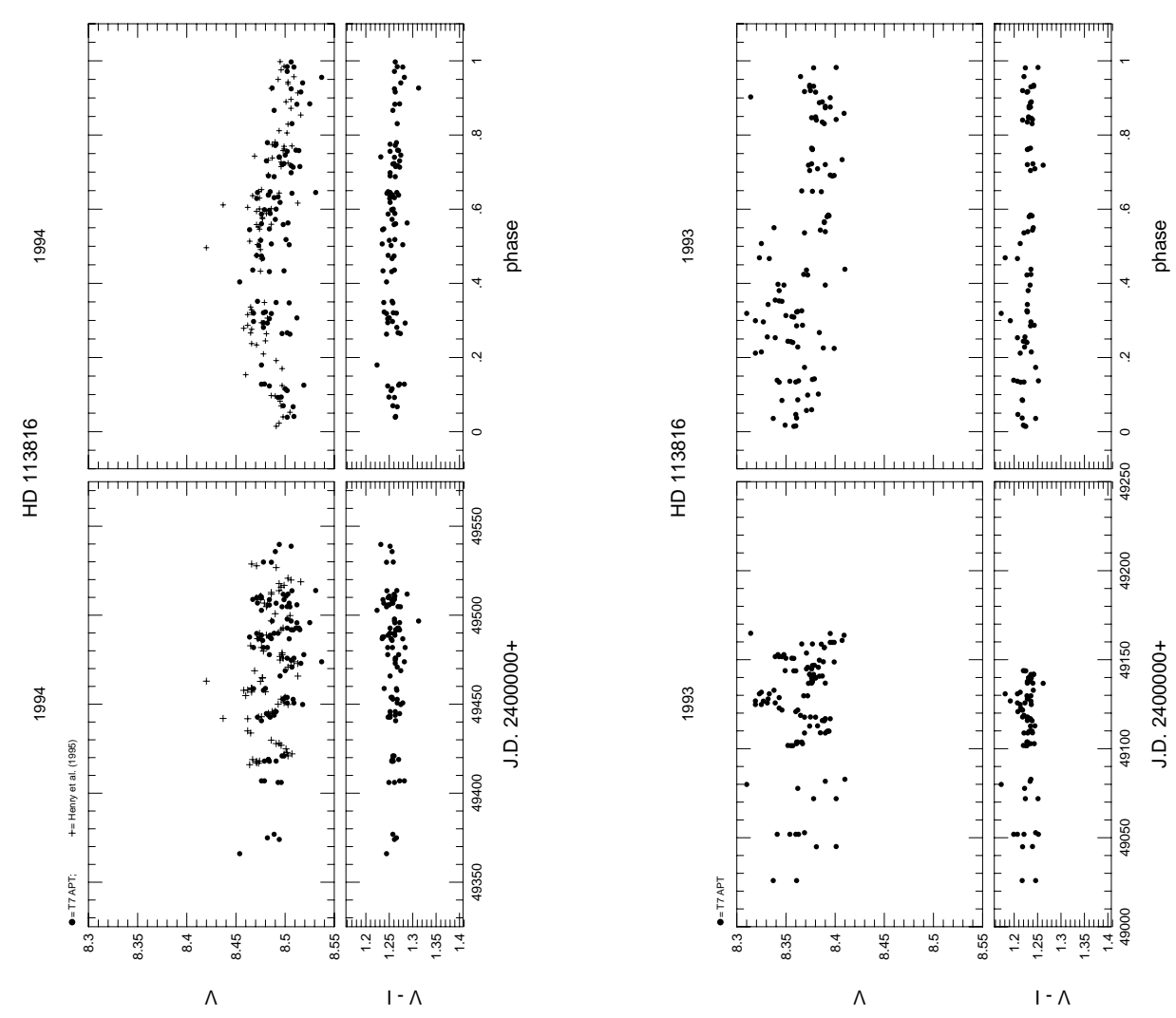

Fig. 41. continued
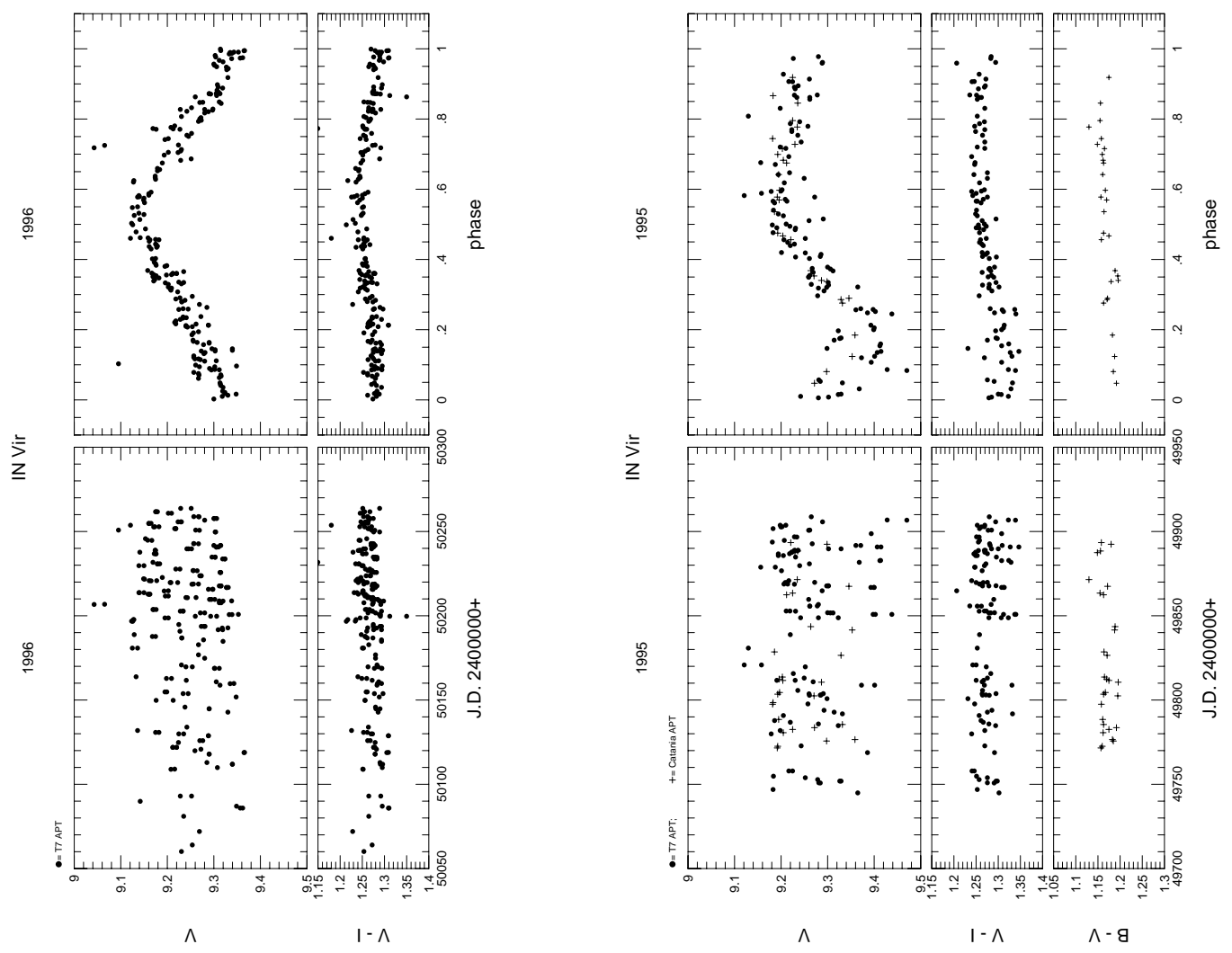

Fig. 42. HD $116544=$ IN Vir 


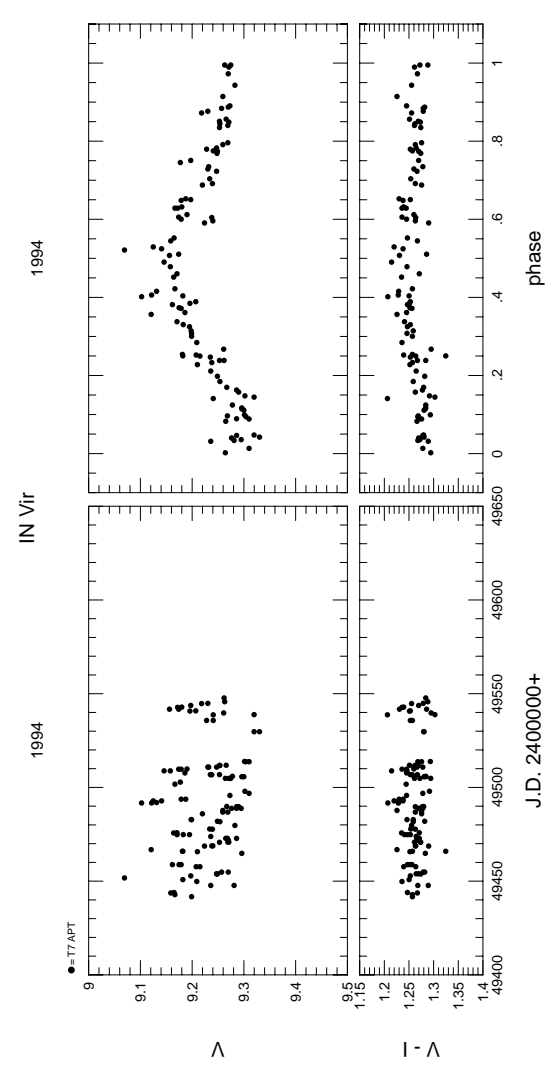

Fig. 42. continued
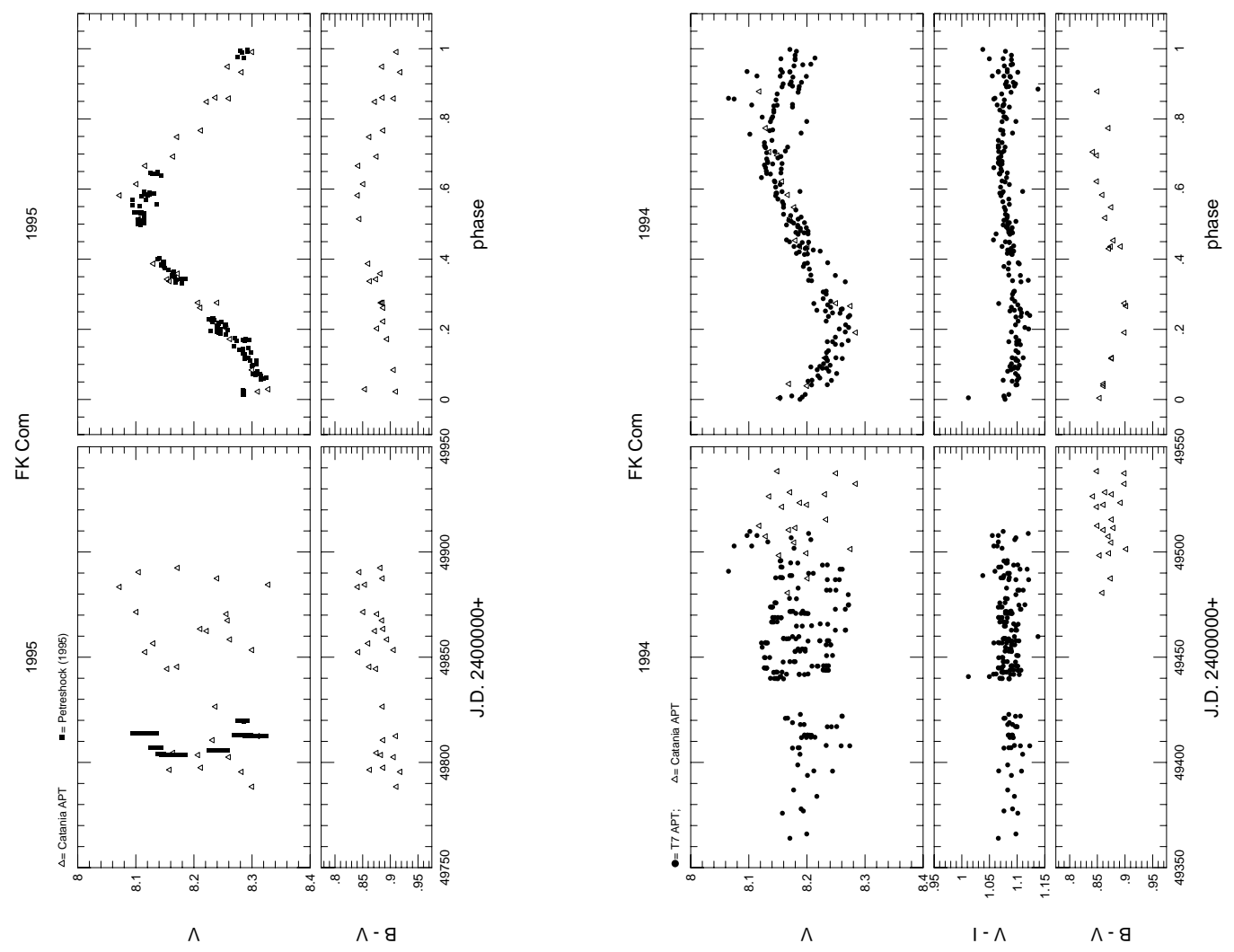

Fig. 43. HD $117555=$ FK Com 


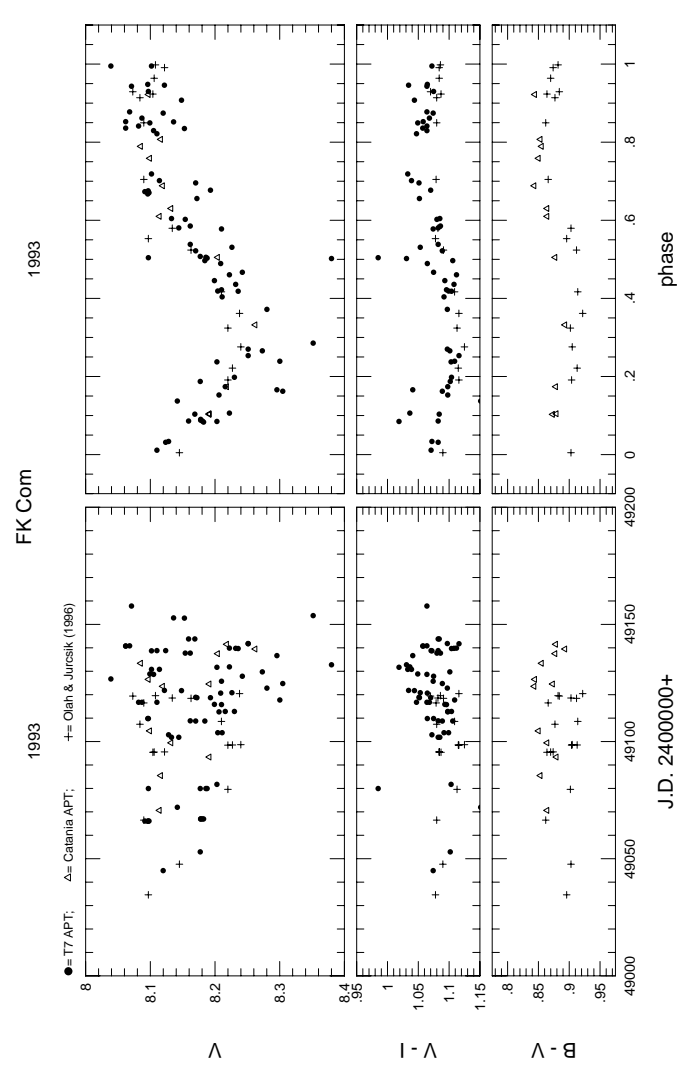

Fig. 43. continued
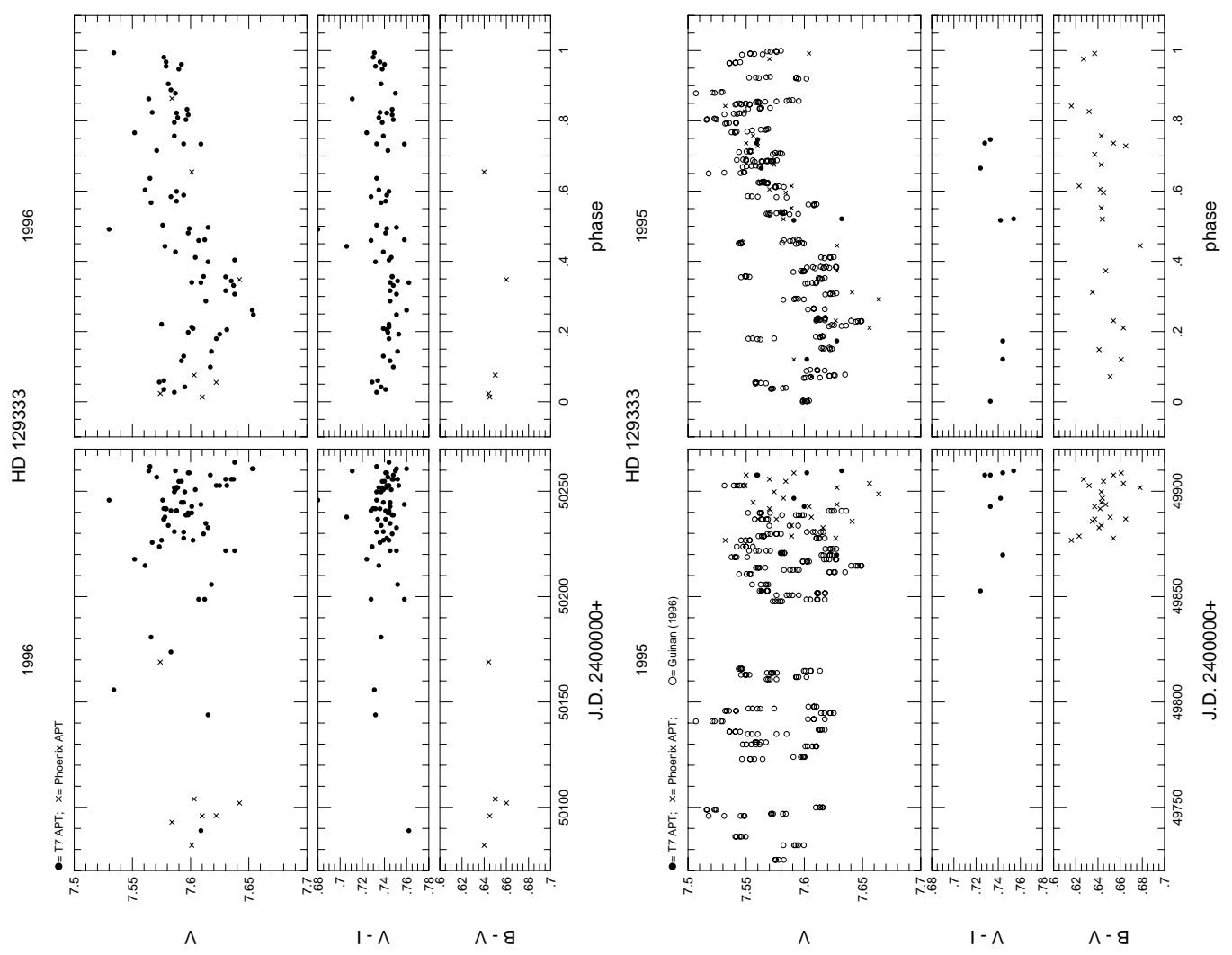

Fig. 44. HD $129333=$ EK Dra 


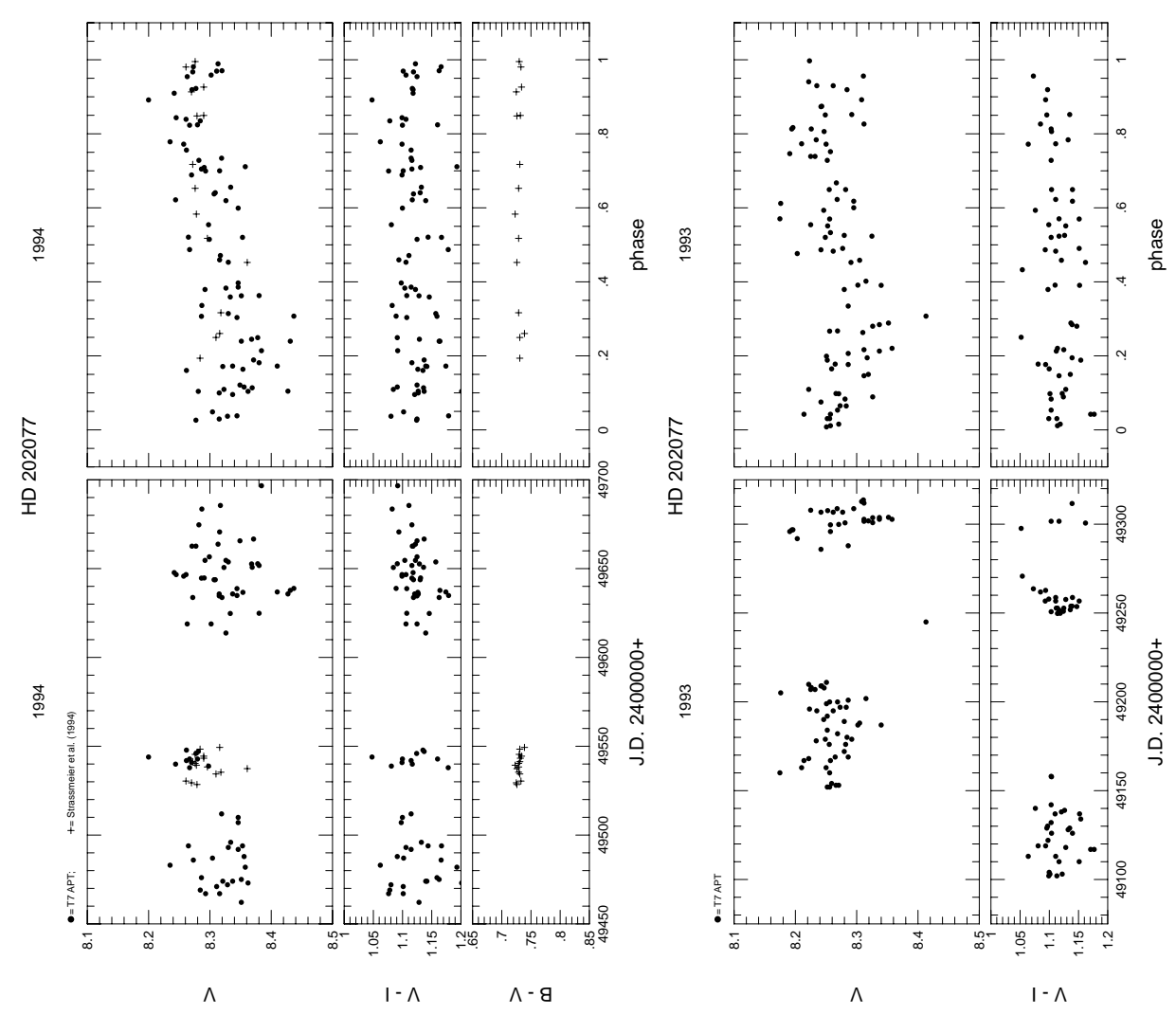

Fig. 47. continued
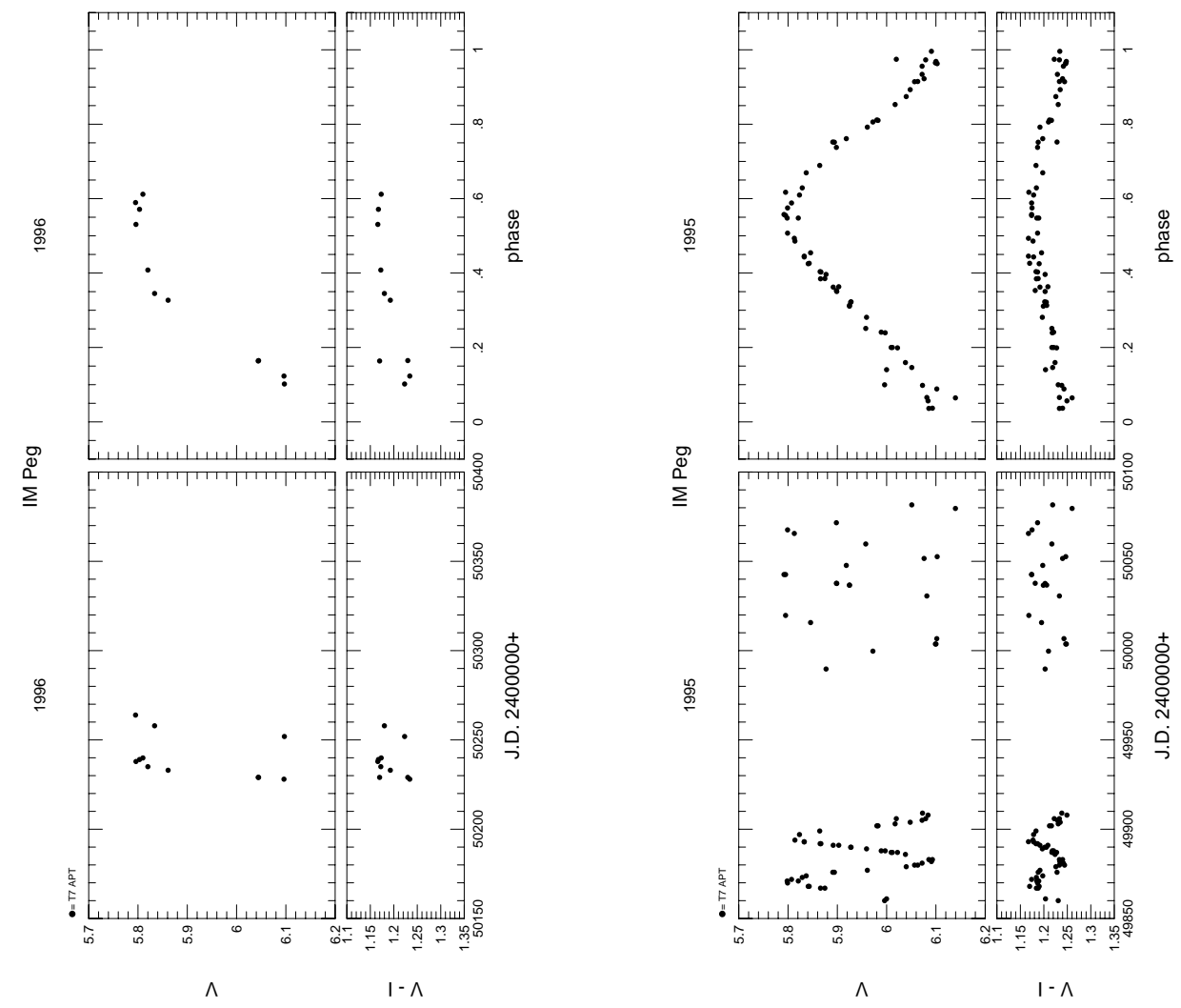

Fig. 48. HD $216489=\mathrm{IM}$ Peg 
